\title{
Synthesis of a Biomimetic Tetracyclic Precursor of Aspochalasins Allowing a Formal Synthesis of Trichoderone A
}

Oscar Gayraud, ${ }^{\mathrm{a}, \#}$ Benjamin Laroche, ${ }^{\mathrm{b}, \#}$ Nicolas Casaretto, ${ }^{\mathrm{c}}$ and Bastien Nay,,a,b

\#Equal contribution

a Laboratoire de Synthèse Organique, Ecole Polytechnique, CNRS, ENSTA, Institut Polytechnique de Paris, Palaiseau 91128, France-E-mail: bastien.nay@polytechnique.edu

${ }^{b}$ Unité Molécules de Communication et Adaptation des Microorganismes, Muséum National d'Histoire Naturelle, CNRS, Paris 75005, France

' Laboratoire de Chimie Moléculaire, Ecole Polytechnique, CNRS, Institut Polytechnique de Paris, Palaiseau 91128, France

\section{Graphical abstract}

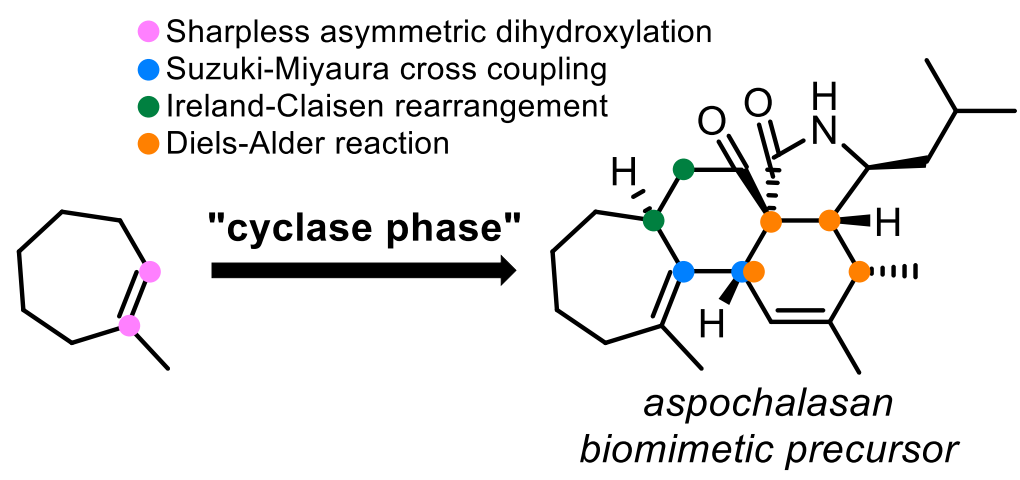

Abstract: Aspochalasins are leucine-derived cytochalasins. Their complexity is often associated to a high degree of biosynthetic oxidative transformations that could inspire a two-phase strategy in total synthesis. In that context, we describe the synthesis of a putative biomimetic tetracyclic intermediate. The key constructive steps are an intramolecular Diels-Alder reaction to install the characteristic isoindolone core of cytochalasins, whose branched precursor was obtained from a stereoselective Ireland-Claisen rearrangement made on a highly unsaturated substrate. This work also constitutes a formal synthesis of trichoderone $A$. 
The bio-inspired two-phase synthesis paradigm first relies on the construction of a minimally oxidized natural product skeleton, often referred to as the "cyclase phase". ${ }^{1}$ This intermediate is then engaged in a second stage involving functional decorations, also called "oxidation phase" when it comes to selective oxidative functionalization, which aims to produce the final natural products. Highly complex natural products have been achieved by this strategy, ${ }^{1-9}$ with the two-phase synthesis of taxol recently achieved by Baran and co-workers standing as a feat in total synthesis. ${ }^{10}$ These examples most often concern terpenoids, while biosynthetic oxidative functionalizations are ubiquitous in natural products.

Cytochalasins obey the same two-phase biosynthetic logic. ${ }^{11,12}$ A core skeleton is first enzymatically constructed by a hybrid polyketide synthase-non-ribosomal peptide synthetase (PKS-NRPS) assembly line,,$^{13,14}$ and then cyclized before additional oxidative functionalizations to deliver the final natural products. ${ }^{15,16} \mathrm{~A}$ two-phase bio-inspired synthetic strategy to synthesize complex cytochalasins or structurally related analogues is thus conceivable if we can efficiently access an appropriate, poorly functionalized precursor.

Owing to their reputed biological activities (e.g. as inhibitors of actin polymerization, ${ }^{17}$ glucose transporters, ${ }^{18}$ or as cytotoxic compounds ${ }^{19}$ ), cytochalasins have often been the target of total synthesis. ${ }^{20}$ More specifically, aspochalasins (e.g. 1) $)^{21}$ are 11 -membered macrocyclic compounds incorporating the typical fused isoindolone heterocycle of cytochalasins (Figure 1). They are biologically derived from l-leucine, as betrayed by the isobutyl substituent. During the biosynthesis, the transannular cyclization of the macrocycle leads to tetracyclic derivatives with a 7/6/6/5 fused system and oxygenated decorations, like aspergillin PZ (2) ${ }^{22}$ recently synthesized by Trauner though a biomimetic cyclization strategy. ${ }^{200}$ An important array of oxidative functionalizations can be encountered at various positions of this carbocyclic skeleton..$^{23}$ Interestingly, oxygen-bridged trichoderone A $(3)^{24}$ and oxidatively cleaved trichodermone $(4)^{25}$ could be derived from a common biosynthetic intermediate (5) after late-stage oxidative functionalizations (Figure 1), with $\mathbf{5}$ possibly arising from biosynthetic precursor 6 after transannular cyclization. This retrobiosynthetic hypothesis differs from the previously suggested route that makes aspochalasin $D(\mathbf{1})$ a precursor of $\mathbf{3}$ and $\mathbf{4}$, through deoxygenation. ${ }^{20 q, 25}$

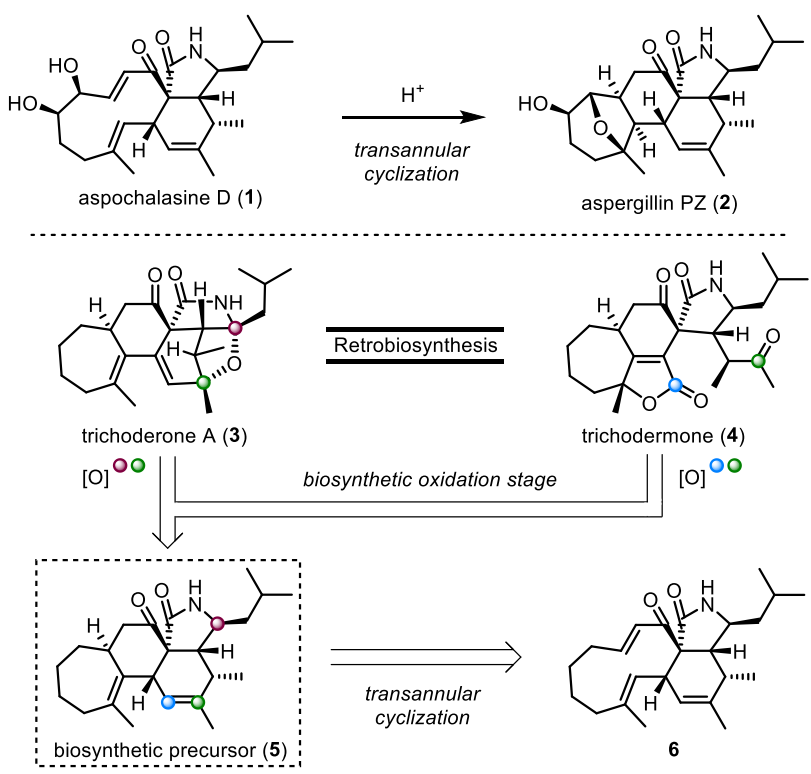

Figure 1. Structure of aspochalasin-related natural products 1-4 and retrobiosynthetic analysis of $\mathbf{3}$ and 4 highlighting precursor 5 for two-phase biomimetic synthesis. 
A two-phase biomimetic strategy towards complex aspochalasins and bio-inspired analogues, involving extensive oxidations, could thus be envisaged if precursor 5 can be synthesized, as we report herein. After the recent impressive work of Puno, Deng and co-workers on the divergent synthesis of aspochalasins, ${ }^{20 q}$ our work also constitutes a formal synthesis of trichoderone A (3). Above all, it provides the preliminary "cyclase" phase for future prospects on the second "oxidase" phase towards functionalized derivatives.

Retrosynthetically, compound $\mathbf{5}$ could be obtained by an intramolecular Diels-Alder reaction (IMDA), expectedly favoring the endo cycloadduct. The dienophile in IMDA substrate $\mathbf{7}$ can be installed in a two-stage manner involving the $\alpha$-acylation of lactam $\mathbf{8}$ with carboxylic acid $\mathbf{9}$ after activation as a mixed anhydride, followed by a selenide-mediated oxidation of the $\alpha$-position. Lactam $\mathbf{8}$ is accessible on a decagram scale from I-leucine. ${ }^{26}$ Compound 9 could be stereoselectively delivered by an IrelandClaisen rearrangement ${ }^{27}$ of allylic acetate 10 , enabling the transfer of stereochemistry from the chiral acetate. ${ }^{28}$ In fact, this allylic acetate is also a sensitive triene and as far as we know, the Ireland-Claisen rearrangement has never been applied to such highly unsaturated substrate. This reaction would however allow an asymmetric access to acid 9, foreseeing an enantioselective synthesis of acetate precursor 10 . The triene moiety could be installed on enol triflate 12 by a Suzuki-Miyaura $s p^{2}-s p^{2}$ crosscoupling ${ }^{29}$ with dienyl boronate $\mathbf{1 1}$. Finally, intermediate $\mathbf{1 2}$ could be synthesized from enantioenriched syn-diol 13, which was envisioned to be obtained from 1-methylcycloheptene 14 through a Sharpless asymmetric dihydroxylation. ${ }^{30}$ This reaction was expected to provide a reliable entry to secure an asymmetric synthesis of compound $\mathbf{5}$.

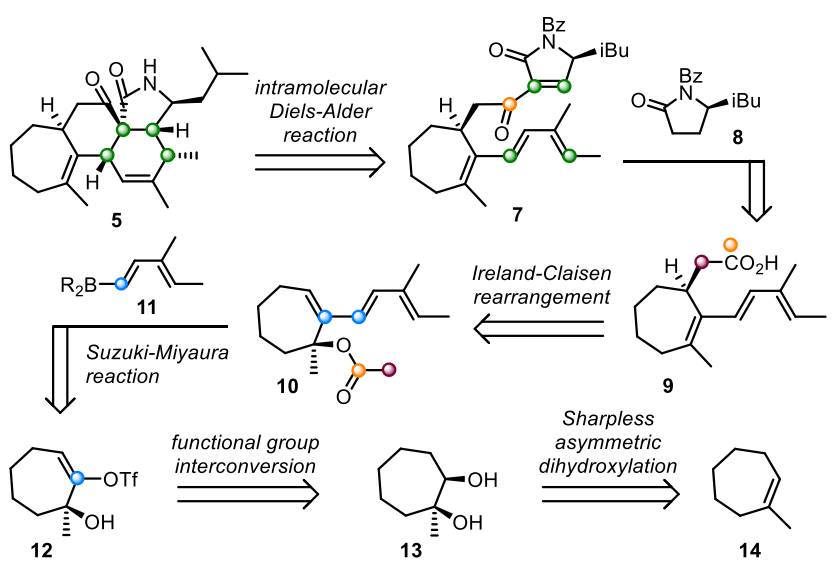

Scheme 1. Retrosynthetic strategy towards intermediate 5.

1-Methylcycloheptene $\mathbf{1 4}$ was available from a two-step literature sequence starting from commercial cycloheptanone. ${ }^{31}$ It was submitted to a Sharpless asymmetric dihydroxylation to access diol $\mathbf{1 3}$ (Scheme 2). Since this reaction had not been described on alkene 14 , we relied on ligand guidelines to choose the most appropriate conditions, ${ }^{32}$ and on the reported dihydroxylation of 1 methylcyclohexene or 1-phenylcycloheptene using the ligand (DHQD) ${ }_{2}$ PHAL [dihydroquinidine-1,4phthalazinediyl diether]. ${ }^{33}$ In the presence of commercially available AD-mix- $\beta$ (involving 1 mol\% of (DHQD) ${ }_{2} \mathrm{PHAL}$ and 0.4 mol\% of $\mathrm{K}_{2} \mathrm{OsO}_{4}$ ) combined with 1 equivalent of $\mathrm{CH}_{3} \mathrm{SO}_{2} \mathrm{NH}_{2}$ in a 1:1 mixture of $t \mathrm{BuOH} / \mathrm{H}_{2} \mathrm{O}$ at room temperature, diol $\mathbf{1 3}$ was promisingly obtained in $65 \%$ yield and an e.r. of 84:16 after 3 days, ${ }^{34}$ which were improved to $69 \%$ and 90:10 at $0^{\circ} \mathrm{C}$ after 7 days. The anthraquinone ligand $(\mathrm{DHQD})_{2} \mathrm{AQN}$ [dihydroquinidine anthraquinone-1,4-diyl diether], also proposed for trisubstituted olefins, was evaluated giving a better NMR yield (88\%) but a lower e.r. $(86: 14)$. Finally, increasing the loadings of $\mathrm{K}_{2} \mathrm{OsO}_{4}(0.7 \mathrm{~mol} \%)$ and of (DHQD) ${ }_{2} \mathrm{PHAL}(1.75 \mathrm{~mol} \%)$ resulted in good yields (81\%) and e.r. (90:10) after 4 days. The reaction was easily reproducible and scalable (5-gram batches). 


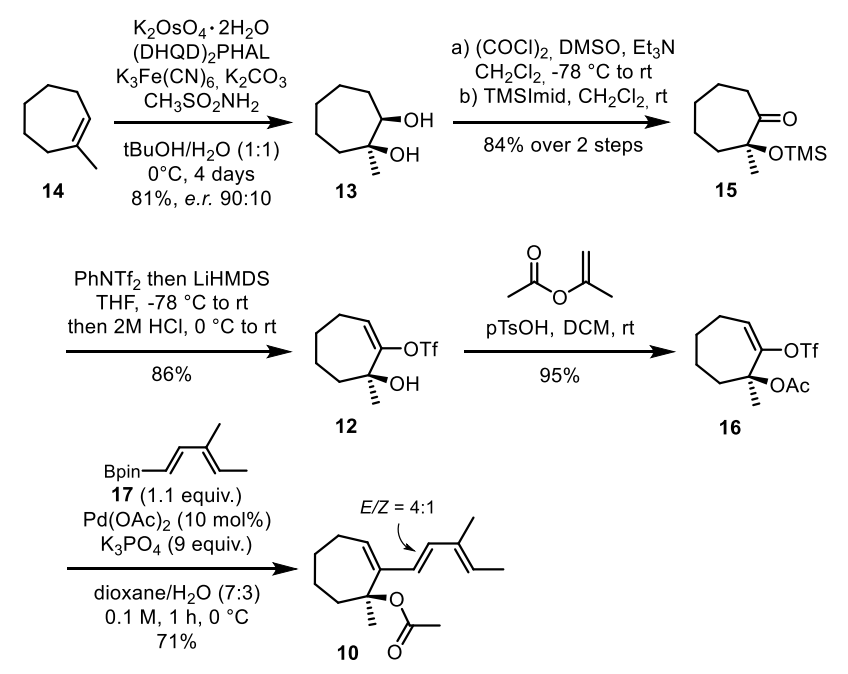

Scheme 2. Synthesis of Ireland-Claisen substrate 10.

Diol 13 was then engaged in functional group manipulation towards enol triflate $\mathbf{1 6}$ (Scheme 2). The oxidation of the secondary alcohol was achieved by a Swern protocol, directly followed by the protection of the tertiary alcohol ${ }^{35}$ in presence of 1-(trimethylsilyl)imidazole (TMSImid), affording 15 in $84 \%$ yield over two steps (11-gram scale). The enol triflation of $\mathbf{1 5}$ was performed by deprotonation with LiHMDS in presence of $\mathrm{PhNTf}_{2}$. The tertiary alcohol was regenerated upon acidic treatment $(\mathrm{HCl})$ at the end of the reaction, giving unprotected enol triflate 12 in 86\% (5-gram scale). Significant difficulties were observed with the acetylation of the tertiary alcohol, in view of the Ireland-Claisen rearrangement. The reaction was ineffective in most classical conditions, but finally succeeded in presence of isopropenyl acetate under acid catalysis ( $p \mathrm{TsOH}$ ), to give acetate 16 in $95 \%$ (1.4 g scale). Unfortunately, these conditions were hardly scalable, the yields dropping gradually from $95 \%$ to $52 \%$ when scaling-up from $1.4 \mathrm{~g}$ to $10 \mathrm{~g}$ (this problem was solved by running the reaction in multiple flasks).

Finally, the next step to Ireland-Claisen substrate $\mathbf{1 0}$ involved a Suzuki-Miyaura cross coupling between triflate $\mathbf{1 6}$ and dienylboronate $\mathbf{1 7}$ (easily available from tiglic aldehyde through a boron-Wittig strategy recently reported by Morken $\left.{ }^{36}\right)$. The cross coupling performed well in the presence of $\mathrm{Pd}(\mathrm{OAc})_{2}(10$ mol\%) and $\mathrm{K}_{3} \mathrm{PO}_{4}$ in a 7:3 dioxane/water mixture at $0{ }^{\circ} \mathrm{C}$ without ligand, providing triene 10 in $71 \%$ yield after one hour. In fact, the absence of ligand allowed to perform the reaction on a $1.6 \mathrm{~g}$ scale, providing 10 as a $4: 1$ mixture of $E$ and $Z$ isomers, respectively, supposedly formed through triene isomerization under reaction conditions. The undesired isomer could be separated during the next step. Although electron-rich ligands like $\mathrm{PCy}_{3}, \mathrm{P}(2 \text {-furyl })_{3}$ or bis(diphenylphosphino)ferrocene (dppf) allowed the reaction to proceed in good yields $(75-80 \%)$, these conditions were hardly reproducible when scaling up the reaction.

Only a few examples of Ireland-Claisen rearrangements have been performed on branched allylic systems bearing conjugated substituents at position 2, like acetate 10. In 2006, Diver and co-workers were able to perform tandem enyne metatheses giving products with the branched 2-vinylallylic acetate topology like ours, which were engaged in the Ireland-Claisen rearrangement. ${ }^{37}$ We first attempted this rearrangement on simplified substrate $\mathbf{1 8}$ previously obtained in a racemic form from enyne metathesis ${ }^{38}$ (Scheme 3). Rearranged product 19 was successfully obtained in $70 \%$ yield after

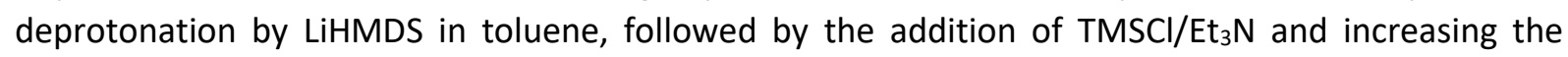
temperature to reflux. Diene $\mathbf{1 9}$ could also be engaged in additional transformations towards triene $\mathbf{9}$ (racemic form), through a sequence of cross metathesis with pinacol vinylboronate and a SuzukiMiyaura cross-coupling with iodoolefin $\mathbf{2 1}$ (available from the corresponding bromoolefin by applying Klapars and Buchwald procedure ${ }^{39}$ ). 


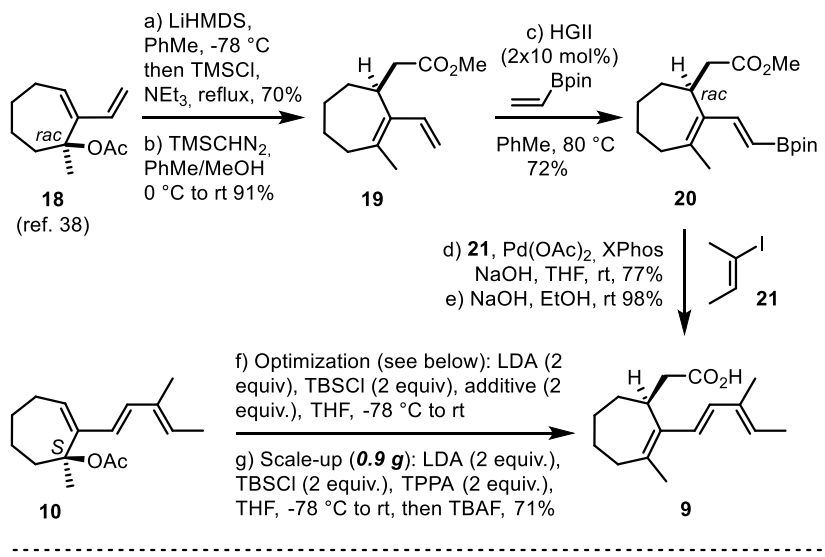

Condition $\mathrm{f}$ : effect of additives on the Ireland-Claisen rearrangement of 10 into 9 (NMR yields with 1,2-dichloroethane as internal standard)

Scheme 3. The Ireland-Claisen rearrangement of branched allylic acetates $\mathbf{1 0}$ and $\mathbf{1 8 .}$

These preliminary results validated our rearrangement strategy, but the conditions were not directly transposable to triene substrate 10. A screening of new conditions in presence of various bases (LiHMDS, LDA or LiTMP) and silyl reagents (TMSCl or TBSCI) in THF showed that in the absence of any additive, the couple LiTMP/TBSCl was the best to afford partial conversion of starting material 10, giving $32 \%$ of 9 . In fact, the use of an additive was crucial for the success of the reaction in presence of LDA. HMPA and the less toxic TPPA (Scheme 3) were the most effective, furnishing 9 in 90 and $84 \%$ yields, respectively. The rearrangement performed well at room temperature in THF. Although the carboxylic acid could be directly obtained on small-scale reactions $(0.2 \mathrm{mmol})$, the same conditions on a larger scale $(3.6 \mathrm{mmol})$ afforded a mixture of the TBS ester and the acid, which needed an additional treatment with TBAF, leading to acid 9 in $71 \%$.

Carboxylic acid 9 was next coupled to $\mathrm{Y}$-lactam $\mathbf{8}$ (Scheme 4), which is available in 5 steps from $\mathrm{N}$-BocI-leucine. ${ }^{26}$ To do so, 9 was activated as a mixed pivaloyl anhydride and added to deprotonated, benzoyl-protected, $\gamma$-lactam 8, giving $\alpha$-acyl- $\gamma$-lactam 22 in 58\% (two steps). These conditions proved superior to the more classical activation of $\mathbf{9}$ as an acyl imidazole by treatment with carbonyldiimidazole. ${ }^{20}$ To install the dienophile, intermediate $\mathbf{2 2}$ was converted into selenide $\mathbf{2 3}$ in $95 \%$ yield by deprotonation in presence of LiHMDS and reaction with PhSeBr. Oxidation and spontaneous elimination in presence of $m$-CPBA and $\mathrm{NaHCO}_{3}$ in $\mathrm{CH}_{2} \mathrm{Cl}_{2}$ at $-78{ }^{\circ} \mathrm{C}$ released the IMDA substrate 7, which was too unstable to be isolated. After reductive and basic treatment of this reaction mixture to eliminate any trace of oxidant and acid, a solution of $\mathbf{7}$ in $\mathrm{CH}_{2} \mathrm{Cl}_{2}$ was heated at $100^{\circ} \mathrm{C}$ in a sealed tube, in presence of $\mathrm{BHT}$ as a radical scavenger, to furnish endo cycloadduct $\mathbf{2 4}$ in $38 \%$ yield and exo product 25 in $27 \%$ yield after purification. It was not possible to improve this reaction, especially by any catalytic process involving a Lewis acid or the Schreiner's catalyst as previously reported for periconiasin derivatives. ${ }^{19}$ Finally, the lactam of cycloadduct $\mathbf{2 4}$ was deprotected under hydrolytic conditions to achieve the synthesis of biomimetic tetracyclic precusor 5 . 

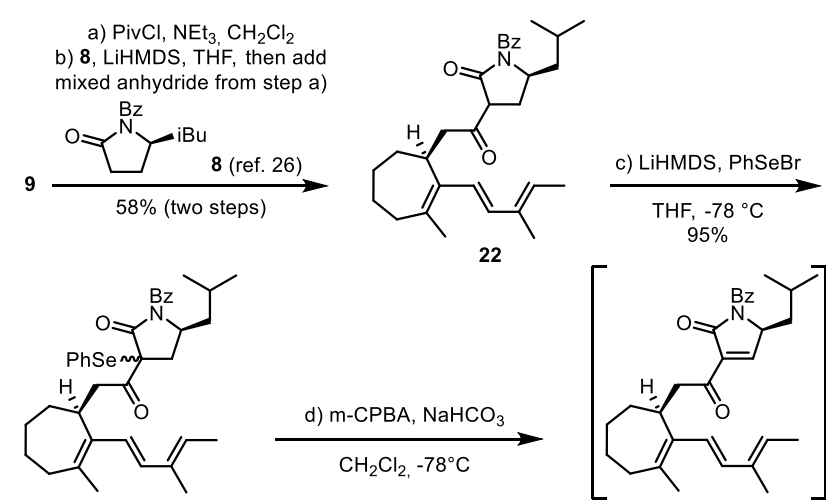

23
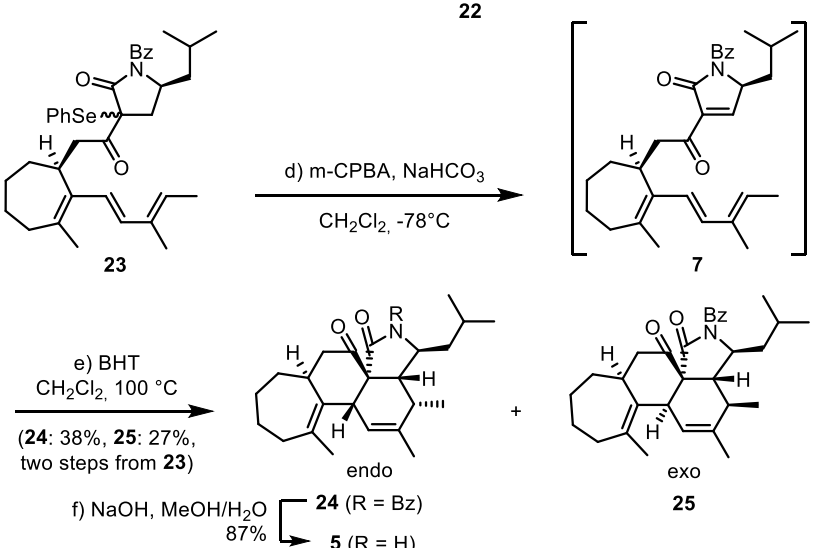

25

Scheme 4. Final steps towards tetracyclic precursor $\mathbf{5}$.

The epoxidation of $\mathbf{2 4}$ could selectively be achieved on the cycloheptene ring, furnishing a 2:1 stereoisomeric mixture of separable epoxides 26 (48\%) and $\mathbf{2 7}$ (22\%). Major isomer 26 ( $\alpha$-epoxide) could be crystalized for X-ray analysis (Scheme 5). Above all, based on Puno's and Deng's recent work $^{20 a}$, this new synthetic strategy provides a formal synthesis of trichoderone $A(3)$, which can be formed from 5 upon air oxidation, through allylic peroxidation and oxidative cyclization.

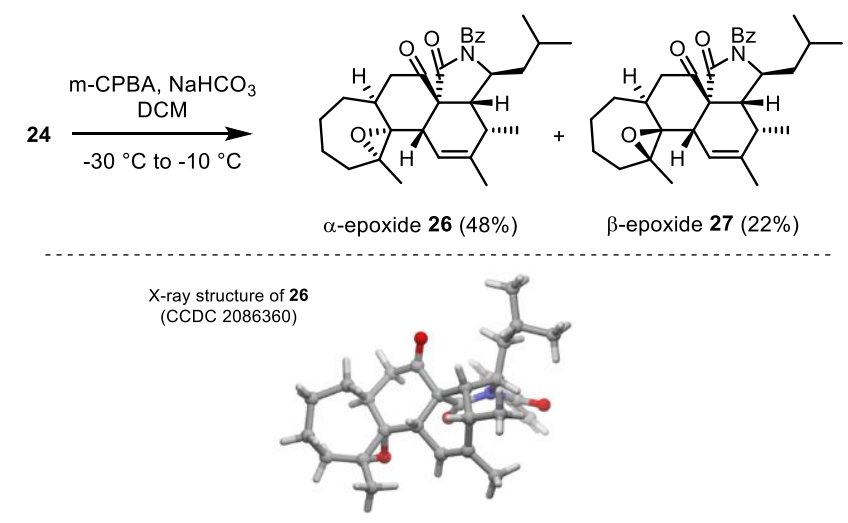

Scheme 5. Epoxidation of $\mathbf{2 4}$ furnishing suitable crystals of $\mathbf{2 6}$ for X-ray analysis.

In conclusion, we were able to perform the synthesis of a key synthetic precursor (5) of tetracyclic aspochalasan natural products and derivatives, the structure of which could be confirmed by X-ray analysis of an epoxide product (26). Compound $\mathbf{5}$ constitutes a good entry to perform the "oxidase" phase in the bio-inspired synthesis of natural products such as trichoderone A (3) and trichodermone (4). In particular, this work constitutes a formal synthesis of 3. ${ }^{20 q}$ Additional oxidations can be envisaged on precursor $\mathbf{5}$, either in a total synthesis perspective, or to synthesize a bio-inspired diversity of aspochalasins for biological studies.

\section{Supporting Information}

Synthetic procedures and copies of NMR spectra (PDF)

Crystal analysis of 26 (CIF) 
CCDC 2086360 (26) contains the supplementary crystallographic data for this paper. These data can be obtained free of charge via www.ccdc.cam.ac.uk/data_request/cif, or by emailing data_request@ccdc.cam.ac.uk, or by contacting The Cambridge Crystallographic Data Centre, 12 Union Road, Cambridge CB2 1EZ, UK; fax: +44 1223336033.

\section{Acknowledgement}

We thank the MESR and the CNRS (Fondation pour le Développement de la Chimie des Substances Naturelles et ses Applications) for providing PhD fellowships to OG and BL. We thank the ANR for funding parts of this project (grants numbers ANR-12-BS07-0028-01 and ANR-19-CE07-0012), and Ecole Polytechnique and CNRS for financial supports.

\section{References}

(1) (a) Chen, K.; Baran, P. S. Total Synthesis of Eudesmane Terpenes by Site-Selective C-H Oxidations. Nature 2009, 459, 824-828; (b) Chen, K.; Ishihara, Y.; Galán, M. M.; Baran, P. S. Total Synthesis of Eudesmane Terpenes: Cyclase Phase. Tetrahedron 2010, 66, 4738-4744; (c) Ishihara, Y.; Baran, P. S. Two-Phase Terpene Total Synthesis: Historical Perspective and Application to the Taxol ${ }^{\circledR}$ Problem. Synlett 2010, 1733-1745.

(2) Wilde, N. C.; Isomura, M.; Mendoza, A.; Baran, P. S. Two-Phase Synthesis of (-)-Taxuyunnanine D. J. Am. Chem. Soc. 2014, 136, 4909-4912.

(3) Jin, Y.; Yeh, C.-H.; Kuttruff, C. A.; Jørgensen, L.; Dünstl, G.; Felding, J.; Natarajan, S. R.; Baran, P. S. $\mathrm{C}-\mathrm{H}$ Oxidation of Ingenanes Enables Potent and Selective Protein Kinase C Isoform Activation. Angew. Chem. Int. Ed. 2015, 54, 14044-14048.

(4) Yuan, C.; Jin, Y.; Wilde, N. C.; Baran, P. S. Short, Enantioselective Total Synthesis of Highly Oxidized Taxanes. Angew. Chem. Int. Ed. 2016, 55, 8280-8284.

(5) Chu, H.; Smith, J. M.; Felding, J.; Baran, P. S. Scalable Synthesis of (-)-Thapsigargin. ACS Cent. Sci. 2017, 3, 47-51.

(6) Kuroda, Y.; Nicacio, K. J.; da Silva-Jr, I. A.; Leger, P. R.; Chang, S.; Gubiani, J. R.; Deflon, V. M.; Nagashima, N.; Rode, A.; Blackford, K.; Ferreira, A. G.; Sette, L. D.; Williams, D. E.; Andersen, R. J.; Jancar, S.; Berlinck, R. G. S.; Sarpong, R. Isolation, Synthesis and Bioactivity Studies of Phomactin Terpenoids. Nature Chem. 2018, 10, 938-945.

(7) Hung, K.; Condakes, M. L.; Novaes, L. F. T.; Harwood, S. J.; Morikawa, T.; Yang, Z.; Maimone, T. J. Development of a Terpene Feedstock-Based Oxidative Synthetic Approach to the Illicium Sesquiterpenes. J. Am. Chem. Soc. 2019, 141, 3083-3099.

(8) Wein, L. A.; Wurst, K.; Angyal, P.; Weisheit, L.; Magauer, T. Synthesis of (-)-Mitrephorone A via a Bioinspired Late Stage C-H Oxidation of (-)-Mitrephorone B. J. Am. Chem. Soc. 2019, 141, 1958919593.

(9) Berger, M.; Knittl-Frank, C.; Bauer, S.; Winter, G.; Maulide, N. Application of Relay C-H Oxidation Logic to Polyhydroxylated Oleanane Triterpenoids. Chem 2020, 6, 1183-1189.

(10) (a) Kanda, Y.; Ishihara, Y.; Wilde, N. C.; Baran, P. S. Two-Phase Total Synthesis of Taxanes: Tactics and Strategies. J. Org. Chem. 2020, 85, 10293-10320; (b) Kanda, Y.; Nakamura, H.; Umemiya, S.; Puthukanoori, R. K.; Murthy Appala, V. R.; Gaddamanugu, G. K.; Paraselli, B. R.; Baran, P. S. Two-Phase Synthesis of Taxol. J. Am. Chem. Soc. 2020, 142, 10526-10533. 
(11) Scherlach, K.; Boettger, D.; Remme, N.; Hertweck, C. The Chemistry and Biology of Cytochalasans. Nat. Prod. Rep. 2010, 27, 869-886.

(12) Skellam, E. The Biosynthesis of Cytochalasans. Nat. Prod. Rep. 2017, 34, 1252-1263.

(13) Fujii, R.; Minami, A.; Gomi, K.; Oikawa, H. Biosynthetic Assembly of Cytochalasin Backbone. Tetrahedron Lett. 2013, 54, 2999-3002.

(14) (a) Song, Z.; Bakeer, W.; Marshall, J. W.; Yakasai, A. A.; Khalid, R. M.; Collemare, J.; Skellam, E.; Tharreau, D.; Lebrun, M.-H.; Lazarus, C. M.; Bailey, A. M.; Simpson, T. J.; Cox, R. J. Heterologous Expression of the Avirulence Gene ACE1 from the Fungal Rice Pathogen Magnaporthe Oryzae. Chem. Sci. 2015, 6, 4837-4845; (b) Zhang, H.; Hantke, V.; Bruhnke, P.; Skellam, E. J.; Cox, R. J. Chemical and Genetic Studies on the Formation of Pyrrolones During the Biosynthesis of Cytochalasans. Chem. Eur. J. 2021, 27, 3106-3113.

(15) Ishiuchi, K.; Nakazawa, T.; Yagishita, F.; Mino, T.; Noguchi, H.; Hotta, K.; Watanabe, K. Combinatorial Generation of Complexity by Redox Enzymes in the Chaetoglobosin A Biosynthesis. J. Am. Chem. Soc. 2013, 135, 7371-7377.

(16) Hu, Y.; Dietrich, D.; Xu, W.; Patel, A.; Thuss, J. A. J.; Wang, J.; Yin, W.-B.; Qiao, K.; Houk, K. N.; Vederas, J. C.; Tang, Y. A Carbonate-Forming Baeyer-Villiger Monooxygenase. Nature Chem. Biol. 2014, $10,552-554$.

(17) Cooper, J. A. Effects of Cytochalasin and Phalloidin on Actin. J. Cell Biol. 1987, 105, 1473-1478.

(18) Carruthers, A.; Helgerson, A. L. Inhibitions of Sugar Transport Produced by Ligands Binding at Opposite Sides of the Membrane. Evidence for Simultaneous Occupation of the Carrier by Maltose and Cytochalasin B. Biochemistry 1991, 30, 3907-3915.

(19) Zaghouani, M.; Gayraud, O.; Jactel, V.; Prévost, S.; Dezaire, A.; Sabbah, M.; Escargueil, A.; Lai, T.L.; Le Clainche, C.; Rocques, N.; Romero, S.; Gautreau, A.; Blanchard, F.; Frison, G.; Nay, B. Multifaceted Study on a Cytochalasin Scaffold: Lessons on Reactivity, Multidentate Catalysis, and Anticancer Properties. Chem. Eur. J. 2018, 24, 16686-16691.

(20) (a) Stork, G.; Nakahara, Y.; Nakahara, Y.; Greenlee, W. J. Total Synthesis of Cytochalasin B. J. Am. Chem. Soc. 1978, 100, 7775-7777; (b) Stork, G.; Nakamura, E. A Simplified Total Synthesis of Cytochalasins via an Intramolecular Diels-Alder Reaction. J. Am. Chem. Soc. 1983, 105, 5510-5512; (c) Thomas, E. J.; Whitehead, J. W. F. Cytochalasan Synthesis: Total Synthesis of Cytochalasin H. J. Chem. Soc., Chem. Commun. 1986, 9, 727-728; (d) Dyke, H.; Sauter, R.; Steel, P.; Thomas, E. J. Cytochalasan Synthesis: Total Synthesis of Cytochalasin G. J. Chem. Soc., Chem. Commun. 1986, 18, 1447-1449 (e) Vedejs, E.; Rodgers, J. D.; Wittenberger, S. J. A Sulfur-Mediated Total Synthesis of Zygosporin E. J. Am. Chem. Soc. 1988, 110, 4822-4823; (f) Trost, B. M.; Ohmori, M.; Boyd, S. A.; Okawara, H.; Brickner, S. J. Palladium-Catalyzed Synthesis of Macrocycles. A Total Synthesis of (-)-Aspochalasin B. J. Am. Chem. Soc. 1989, 111, 8281-8284; (g) Merifield, E.; Thomas, E. J. Cytochalasan Synthesis: Total Synthesis of Cytochalasin D. J. Chem. Soc., Chem. Commun. 1990, 6, 464-466; (h) Merifield, E.; Thomas, E. J. Total Synthesis of Cytochalasin D: Total Synthesis and Full Structural Assignment of Cytochalasin O. J. Chem. Soc., Perkin Trans. 1 1999, 22, 3269-3283; (i) Haidle, A. M.; Myers, A. G. An Enantioselective, Modular, and General Route to the Cytochalasins: Synthesis of L-696,474 and Cytochalasin B. PNAS 2004, 101, 12048-12053; (j) Canham, S. M.; Overman, L. E.; Tanis, P. S. Identification of an Unexpected 2Oxonia[3,3]Sigmatropic Rearrangement/Aldol Pathway in the Formation of Oxacyclic Rings. Total Synthesis of (+)-Aspergillin PZ. Tetrahedron 2011, 67, 9837-9843; (k) Tian, C.; Lei, X.; Wang, Y.; Dong, Z.; Liu, G.; Tang, Y. Total Syntheses of Periconiasins A-E. Angew. Chem. Int. Ed. 2016, 55 (24), 6992- 
6996; (I) Zaghouani, M.; Kunz, C.; Guédon, L.; Blanchard, F.; Nay, B. First Total Synthesis, Structure Revision, and Natural History of the Smallest Cytochalasin: (+)-Periconiasin G. Chem. Eur. J. 2016, 22, 15257-15260; (m) Bao, R.; Tian, C.; Zhang, H.; Wang, Z.; Dong, Z.; Li, Y.; Gao, M.; Zhang, H.; Liu, G.; Tang, Y. Total Syntheses of Asperchalasines A-E. Angew. Chem. Int. Ed. 2018, 57, 14216-14220; (n) Long, X.; Ding, Y.; Deng, J. Total Synthesis of Asperchalasines A, D, E, and H. Angew. Chem. Int. Ed. 2018, 57, 14221-14224; (o) Reyes, J. R.; Winter, N.; Spessert, L.; Trauner, D. Biomimetic Synthesis of (+)-Aspergillin PZ. Angew. Chem. Int. Ed. 2018, 57, 15587-15591; (p) Zeng, Z.; Chen, C.; Zhang, Y. Enantioselective Total Synthesis of Periconiasin A. Org. Chem. Front. 2018, 5, 838-840; (q) Wu, H.; Ding, Y.; Hu, K.; Long, X.; Qu, C.; Puno, P.-T.; Deng, J. Bioinspired Network Analysis Enabled Divergent Syntheses and Structure Revision of Pentacyclic Cytochalasans. Angew. Chem. Int. Ed. 2021, doi: 10.1002/anie.202102831.

(21) Keller-Schierlein, W.; Kupfer, E. Stoffwechselprodukte von Mikroorganismen. 186. Mitteilung. Über die Aspochalasine A, B, C und D. Helv. Chim. Acta 1979, 62, 1501-1524.

(22) Zhang, Y.; Wang, T.; Pei, Y.; Hua, H.; Feng, B. Aspergillin PZ, a Novel Isoindole-Alkaloid from Aspergillus Awamori. J. Antibiot. 2002, 55, 693-695.

(23) Wei, G.; Tan, D.; Chen, C.; Tong, Q.; Li, X.-N.; Huang, J.; Liu, J.; Xue, Y.; Wang, J.; Luo, Z.; Zhu, H.; Zhang, Y. Flavichalasines A-M, Cytochalasan Alkaloids from Aspergillus Flavipes. Sci. Rep. 2017, 7, 42434.

(24) Ding, G.; Wang, H.; Li, L.; Chen, A. J.; Chen, L.; Chen, H.; Zhang, H.; Liu, X.; Zou, Z. Trichoderones A and B: Two Pentacyclic Cytochalasans from the Plant Endophytic Fungus Trichoderma Gamsii. Eur. J. Org. Chem. 2012, 2012, 2516-2519.

(25) Ding, G.; Wang, H.; Li, L.; Song, B.; Chen, H.; Zhang, H.; Liu, X.; Zou, Z. Trichodermone, a SpiroCytochalasan with a Tetracyclic Nucleus (7/5/6/5) Skeleton from the Plant Endophytic Fungus Trichoderma Gamsii. J. Nat. Prod. 2014, 77, 164-167.

(26) Smrcina, M.; Majer, P.; Majerová, E.; Guerassina, T. A.; Eissenstat, M. A. Facile Stereoselective Synthesis of $\psi$-Substituted $\gamma$-Amino Acids from the Corresponding $\alpha$-Amino Acids. Tetrahedron 1997, $53,12867-12874$.

(27) Ireland, R. E.; Mueller, R. H. Claisen Rearrangement of Allyl Esters. J. Am. Chem. Soc. 1972, 94, 5897-5898.

(28) (a) Ziegler, F. E. The Thermal, Aliphatic Claisen Rearrangement. Chem. Rev. 1988, 88, 1423-1452; (b) Asymmetric [3.3]-Sigmatropic Rearrangements in Organic Synthesis. Tetrahedron Asym. 1996, 7, 1847-1882; (c) Chai, Y.; Hong, S.; Lindsay, H. A.; McFarland, C.; McIntosh, M. C. New Aspects of the Ireland and Related Claisen Rearrangements. Tetrahedron 2002, 58, 2905-2928.

(29) Miyaura, N.; Suzuki, K. Y. A. A New Stereospecific Cross-Coupling by the Palladium-Catalyzed Reaction of 1-Alkenylboranes with 1-Alkenyl or 1-Alkynyl Halides. Tetrahedron Lett. 1979, 20, 34373440 .

(30) (a) Jacobsen, E. N.; Marko, I..; Mungall, W. S.; Schroeder, G.; Sharpless, K. B. Asymmetric Dihydroxylation via Ligand-Accelerated Catalysis. J. Am. Chem. Soc. 1988, 110, 1968-1970; (b) Sharpless, K. B.; Amberg, W.; Bennani, Y. L.; Crispino, G. A.; Hartung, J.; Jeong, K. S.; Kwong, H. L.; Morikawa, K.; Wang, Z. M. The Osmium-Catalyzed Asymmetric Dihydroxylation: A New Ligand Class and a Process Improvement. J. Org. Chem. 1992, 57, 2768-2771. 
(31) Barbier, M.; Hügel, M. F. Synthèses dans la série de l'acide céto-9 décène-2 trans oïque ("substance royale"). Bull. Soc. Chim. Fr. 1961, 28, 951-954.

(32) Kolb, H. C.; Sharpless K. B. In Transition Metals for Organic Synthesis, Vol. 2; Beller, M.; Bolm, C. Eds.; Wiley-VCH: Weinheim (2004), pp. 275-307.

(33) Kolb, H. C.; VanNieuwenhze, M. S.; Sharpless, K. B. Catalytic Asymmetric Dihydroxylation. Chem. Rev. 1994, 94, 2483-2547.

(34) Enantiomeric ratios (e.r.) were measured by chiral HPLC after benzoylation of the secondary alcohol (see the SI for details).

(35) Any attempt to shorten this route, especially by introducing the acetate group before the enol triflation proved ineffective.

(36) (a) Coombs, J. R.; Zhang, L.; Morken, J. P. Synthesis of Vinyl Boronates from Aldehydes by a Practical Boron-Wittig Reaction. Org. Lett. 2015, 17, 1708-1711; (b) Liu, X.; Deaton, T. M.; Haeffner, F.; Morken, J. P. A Boron Alkylidene-Alkene Cycloaddition Reaction: Application to the Synthesis of Aphanamal. Angew. Chem. Int. Ed. 2017, 56, 11485-11489.

(37) Clark, D. A.; Kulkarni, A. A.; Kalbarczyk, K.; Schertzer, B.; Diver, S. T. Tandem Enyne Metathesis and Claisen Rearrangement: A Versatile Approach to Conjugated Dienes of Variable Substitution Patterns. J. Am. Chem. Soc. 2006, 128, 15632-15636.

(38) Laroche, B.; Detraz, M.; Blond, A.; Dubost, L.; Mailliet, P.; Nay, B. Ring-Closing Enyne Metathesis of Terminal Alkynes with Propargylic Hindrance. J. Org. Chem. 2015, 80, 5359-5363.

(39) Klapars A.; Buchwald, S.L. Copper-Catalyzed Halogen Exchange in Aryl Halides: An Aromatic Finkelstein Reaction. J Am. Chem. Soc. 2002, 124, 14844-14845. 


\section{Supporting Information}

\section{Synthesis of a Biomimetic Tetracyclic Precursor of Aspochalasins Allowing a Formal Synthesis of Trichoderone A}

Oscar Gayraud, Benjamin Laroche, Nicolas Casaretto, and Bastien Nay

\section{Table of content}

$\begin{array}{ll}\text { Experimental procedures } & 3\end{array}$

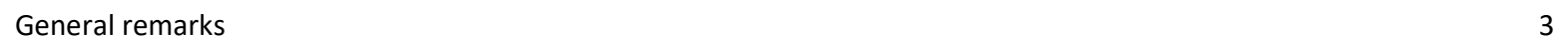

(1S,2R)-1,2-Dihydroxy-1-methylcycloheptane (13) 3

(2S)-2-Trimethylsilyloxy-2-methylcycloheptan-1-one (15) 4

(7S)-7-Hydroxy-7-methyl-1-trifluoromethanesulfonyloxycyclohept-1-ene (12) 5

(7S)-7-Acetoxy-7-methyl-1-trifluoromethanesulfonyloxycyclohept-1-ene (16) 5

(7S)-7-Acetoxy-7-methyl-1-[(1E,3E)-3-methylpenta-1,3-dien-1-yl]cyclohept-1-ene (10), as a 4:1 mixture with the $1 Z$ isomer

( $r a c)$-2-[3-Methyl-2-vinylcyclohept-2-en-1-yl]acetic acid (S2) 6

(rac)-2-[3-Methyl-2-vinylcyclohept-2-en-1-yl]acetic acid, methyl ester (19) 7

(E)-2-(3-Methyl-2-(2-(4,4,5,5-tetramethyl-1,3,2-dioxaborolan-2-yl)vinyl)cyclohept-2-en-1-yl)acetic acid, methyl ester (20)

2-(3-Methyl-2-((1E,3E)-3-methylpenta-1,3-dien-1-yl)cyclohept-2-en-1-yl)acetic acid, methyl ester (S3) 9

(rac)-2-[3-Methyl-2-((1E,3E)-3-methylpenta-1,3-dien-1-yl)cyclohept-2-en-1-yl]acetic acid (rac-9) 9

2-[(1S)-3-Methyl-2-((1E,3E)-3-methylpenta-1,3-dien-1-yl)cyclohept-2-en-1-yl]acetic acid (9) 10

N-Benzoyl-5-isobutyl-3-\{2-[(1S)-3-methyl-2-((1E,3E)-3-methylpenta-1,3-dien-1-yl)cyclohept-2-en-1-yl]acetyl\}-2-pyrrolidone

$\begin{array}{lr}\text { (mixture of stereoisomers 22) } & 10\end{array}$

N-Benzoyl-5-isobutyl-3-\{2-[(1S)-3-methyl-2-((1E,3E)-3-methylpenta-1,3-dien-1-yl)cyclohept-2-en-1-yl]acetyl\}-3-

phenylselenyl-2-pyrrolidone (mixture of stereoisomers 23)

$(3 S, 3 \mathrm{a} R, 4 S, 6 \mathrm{aS}, 8 \mathrm{aS}, 13 \mathrm{~b} R)$-5-Benzoyl-4-isobutyl-2,3,13-trimethyl-4,5,8,8a,9,10,11,12-octahydro-3H-

cyclohepta[3,4]benzo[1,2-d]isoindole-6,7(3aH,13bH)-dione (endo product 24) 12

$(3 R, 3 a R, 4 S, 6 a S, 8 a S, 13 \mathrm{bS})$-5-Benzoyl-4-isobutyl-2,3,13-trimethyl-4,5,8,8a,9,10,11,12-octahydro-3H-

cyclohepta[3,4]benzo[1,2-d]isoindole-6,7(3aH,13bH)-dione (exo product 25) 12

$(3 S, 3 \mathrm{a} R, 4 S, 6 \mathrm{a} S, 8 \mathrm{aS}, 13 \mathrm{~b} R)$-4-Isobutyl-2,3,13-trimethyl-4,5,8,8a,9,10,11,12-octahydro-3H-cyclohepta[3,4]benzo[1,2-

d]isoindole-6,7(3aH,13bH)-dione (5) 13

$(3 S, 3 a R, 4 S, 6 a S, 8 a S, 13 R, 13 a R, 13 b R)$-5-Benzoyl-13,13a-epoxy-4-isobutyl-2,3,13-trimethyl-4,5,8,8a,9,10,11,12-octahydro-3Hcyclohepta[3,4]benzo[1,2-d]isoindole-6,7(3aH,13bH)-dione (26) 13

(3S,3aR,4S,6aS,8aS,13S,13aS,13bR)-5-Benzoyl-13,13a-epoxy-4-isobutyl-2,3,13-trimethyl-4,5,8,8a,9,10,11,12-octahydro-3Hcyclohepta[3,4]benzo[1,2-d]isoindole-6,7(3aH,13bH)-dione (27)

$\begin{array}{lr}\text { X-Ray crystallography of epoxide } 26 & 14\end{array}$

$\begin{array}{lr}\text { Copies of NMR spectra } & 27\end{array}$

$\begin{array}{lr}\text { (1S,2R)-1,2-Dihydroxy-1-methylcycloheptane (13) } & 27\end{array}$

$\begin{array}{lr}\text { (2S)-2-Hydroxy-2-methylcycloheptan-1-one (S1) } & 28\end{array}$

(2S)-2-Trimethylsilyloxy-2-methylcycloheptan-1-one (15) 29

(7S)-7-Hydroxy-7-methyl-1-trifluoromethanesulfonyloxycyclohept-1-ene (12) 30

(7S)-7-Acetoxy-7-methyl-1-trifluoromethanesulfonyloxycyclohept-1-ene (16) 31

(7S)- 7-Acetoxy-7-methyl-1-[(1E,3E)-3-methylpenta-1,3-dien-1-yl]cyclohept-1-ene (10), as a 4:1 mixture with the $1 Z$ isomer

( rac)-2-[3-Methyl-2-vinylcyclohept-2-en-1-yl]acetic acid (S2)

(rac)-2-[3-Methyl-2-vinylcyclohept-2-en-1-yl]acetic acid, methyl ester (19) 34

(E)-2-(3-Methyl-2-(2-(4,4,5,5-tetramethyl-1,3,2-dioxaborolan-2-yl)vinyl)cyclohept-2-en-1-yl)acetic acid, methyl ester (20)

2-(3-Methyl-2-((1E,3E)-3-methylpenta-1,3-dien-1-yl)cyclohept-2-en-1-yl)acetic acid, methyl ester (S3)

(rac)-2-[3-Methyl-2-((1E,3E)-3-methylpenta-1,3-dien-1-yl)cyclohept-2-en-1-yl]acetic acid (rac-9) 38

2-[(1S)-3-Methyl-2-((1E,3E)-3-methylpenta-1,3-dien-1-yl)cyclohept-2-en-1-yl]acetic acid, crude extract used in the next step (9) 
N-Benzoyl-5-isobutyl-3-\{2-[(1S)-3-methyl-2-((1E,3E)-3-methylpenta-1,3-dien-1-yl)cyclohept-2-en-1-yl]acetyl\}-2-pyrrolidone, mixture of stereoisomers (22)

N-Benzoyl-5-isobutyl-3-\{2-[(1S)-3-methyl-2-((1E,3E)-3-methylpenta-1,3-dien-1-yl)cyclohept-2-en-1-yl]acetyl\}-3phenylselenyl-2-pyrrolidone, mixture of stereoisomers (23)

(3S,3aR,4S,6aS,8aS,13bR)-5-Benzoyl-4-isobutyl-2,3,13-trimethyl-4,5,8,8a,9,10,11,12-octahydro-3H-

cyclohepta[3,4]benzo[1,2-d]isoindole-6,7(3aH,13bH)-dione (endo product 24)

$(3 R, 3 a R, 4 S, 6 a S, 8 a S, 13 \mathrm{bS})$-5-benzoyl-4-isobutyl-2,3,13-trimethyl-4,5,8,8a,9,10,11,12-octahydro-3H-

cyclohepta[3,4]benzo[1,2-d]isoindole-6,7(3aH,13bH)-dione (exo product 25)

$(3 S, 3 \mathrm{a} R, 4 S, 6 \mathrm{a} S, 8 \mathrm{a} S, 13 \mathrm{~b} R)$-4-Isobutyl-2,3,13-trimethyl-4,5,8,8a,9,10,11,12-octahydro-3H-cyclohepta[3,4]benzo[1,2-

d] isoindole-6,7(3aH,13bH)-dione (biomimetic precursor 5)

$(3 S, 3 \mathrm{a} R, 4 S, 6 \mathrm{aS}, 8 \mathrm{a} S, 13 R, 13 \mathrm{a} R, 13 \mathrm{~b} R)$-5-Benzoyl-13,13a-epoxy-4-isobutyl-2,3,13-trimethyl-4,5,8,8a,9,10,11,12-octahydro-3Hcyclohepta[3,4]benzo[1,2-d]isoindole-6,7(3aH,13bH)-dione (26) 


\section{Experimental procedures}

\section{General remarks}

All reactions were carried out in oven-dried vessels under an atmosphere of argon and in anhydrous solvents unless stated otherwise. Solvents (methylene chloride, diethyl ether and tetrahydrofuran) were purified using a MB-SPS 800 Solvent purification system (MBraun). Analytical thin-layer chromatography (TLC) was carried out using aluminium TLC plates coated with $\mathrm{F}_{254}$ silica gel 60 (Merck), which were visualized by exposure to ultraviolet light and/or exposure to a basic solution of potassium permanganate or $p$-anisaldehyde stain followed by heating. Flash chromatography was carried out on silica gel $60(40-63 \mu \mathrm{m})$. Chiral HPLC analyses were performed on a Shimadzu Nexera X2 system equipped with a Prominence diode array detector detector (UV-vis).

Infrared spectra were recorded on a PerkinElmer spectrum two FTIR equipped with a Jasco ATR. Absorption maxima $\left(v_{\max }\right)$ are reported in wavenumbers $\left(\mathrm{cm}^{-1}\right)$. High-resolution mass spectra (HRMS) were obtained on a JEOL JMS-GCmate II spectrometer (EI+) or on a Bruker tims-TOF mass spectrometer $(\mathrm{ESI}+)$, and reported as $\mathrm{m} / \mathrm{z}$. Nuclear magnetic resonance spectra $\left({ }^{1} \mathrm{H}-\mathrm{NMR}\right.$ and $\left.{ }^{13} \mathrm{C}-\mathrm{NMR}\right)$ were recorded at $25^{\circ} \mathrm{C}$ with a Brucker Avance 400 or a Bruker $400 \mathrm{MHz}$ Avance III $\left({ }^{1} \mathrm{H}\right.$ at $400 \mathrm{MHz},{ }^{13} \mathrm{C}$ at 100 $\mathrm{MHz}$ ) or a Bruker $600 \mathrm{MHz}$ Avance III spectrometer $\left({ }^{1} \mathrm{H}\right.$ at $600 \mathrm{MHz},{ }^{13} \mathrm{C}$ at $\left.150 \mathrm{MHz}\right)$. Chemical shifts in $\mathrm{CDCl}_{3}$ and $\mathrm{C}_{6} \mathrm{D}_{6}$ solutions are reported as parts per million $(\mathrm{ppm})$ referenced to residual protium or carbon of the solvent (for $\mathrm{CDCl}_{3}: \delta_{H}=7.26$ and $\delta_{C}=77.0$; for $\mathrm{C}_{6} \mathrm{D}_{6}: \delta_{H}=7.16$ and $\delta_{C}=128.1$ ). Coupling constants are reported in Hertz $(\mathrm{Hz})$. Data for ${ }^{1} \mathrm{H}-\mathrm{NMR}$ spectra are reported as follows: chemical shift $\mathrm{ppm}$, referenced to protium $(\mathrm{br}=$ broad, $\mathrm{s}=$ singlet, $\mathrm{d}=$ doublet, $\mathrm{t}=$ triplet, $\mathrm{q}=$ quartet, $\mathrm{dd}=$ doublet of doublets, $\mathrm{dq}=$ doublet of quartets, $\mathrm{td}=$ triplet of doublets, $\mathrm{qq}=$ quartets of quartets, $\mathrm{ddd}=$ doublet of doublet of doublets, $m=$ multiplet, integration, and coupling constants $(\mathrm{Hz})$ ).

\section{(1S,2R)-1,2-Dihydroxy-1-methylcycloheptane (13)}

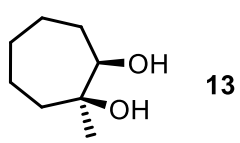

To a stirred solution of $\mathrm{MeSO}_{2} \mathrm{NH}_{2}\left(4.75 \mathrm{~g}, 50 \mathrm{mmol}, 1\right.$ equiv), $\mathrm{K}_{2} \mathrm{CO}_{3}(20.7 \mathrm{~g}, 150 \mathrm{mmol}, 3$ equiv), $\mathrm{K}_{3} \mathrm{Fe}(\mathrm{CN})_{6}\left(49.3 \mathrm{~g}, 150 \mathrm{mmol}, 3\right.$ equiv), (DHQD) $2 \mathrm{PHAL}$ ( $681 \mathrm{mg}, 0.875 \mathrm{mmol}, 0.0175$ equiv), $\mathrm{K}_{2} \mathrm{OsO}_{4} .2 \mathrm{H}_{2} \mathrm{O}$ (129 mg, $0.350 \mathrm{mmol}, 0.007$ equiv) in $500 \mathrm{~mL}{ }^{t} \mathrm{BuOH} / \mathrm{H}_{2} \mathrm{O}$ (1:1) was added 1-methylcycloheptene 14 ( $5.5 \mathrm{~g}, 50 \mathrm{mmol}, 1$ equiv, prepared according to Barbier and $\mathrm{Hügel}^{1}$ ) at $0^{\circ} \mathrm{C}$. The reaction mixture was stirred for 4 days at $0{ }^{\circ} \mathrm{C}$. The reaction was quenched with $\mathrm{Na}_{2} \mathrm{~S}_{2} \mathrm{O}_{3}(75 \mathrm{~g})$, stirred for $1 \mathrm{~h}$ at $\mathrm{rt}$. The aqueous layer was extracted with EtOAc $(3 \times 300 \mathrm{~mL})$. The organic layers were combined, washed with a $1 \mathrm{M}$ solution of $\mathrm{NaOH}(500 \mathrm{~mL})$, brine, dried over $\mathrm{Na}_{2} \mathrm{SO}_{4}$, filtered and concentrated in vacuo. The crude product was purified via silica gel chromatography (PE/EA 6:1 to $2: 1$ ) to yield $5.92 \mathrm{~g}$ (82\% yield) of diol 13 with an e.r. of 90:10 (see below), as a colorless oil.

$[\alpha]_{\mathrm{D}}^{25}=-4.6\left(\mathrm{c} 0.8, \mathrm{CHCl}_{3}\right)$.

${ }^{1} \mathrm{H}$ NMR $\left(400 \mathrm{MHz}, \mathrm{CDCl}_{3}\right) \delta$ ppm: 3.44 (br d, $\left.1 \mathrm{H}\right), 2.32$ (brs, $\left.1 \mathrm{H}\right), 2.17$ (brs, $\left.1 \mathrm{H}\right), 1.89-1.76(\mathrm{~m}, 2 \mathrm{H}), 1.76-$ $1.66(\mathrm{~m}, 3 \mathrm{H}), 1.65-1.56(\mathrm{~m}, 2 \mathrm{H}), 1.50-1.30(\mathrm{~m}, 3 \mathrm{H}), 1.28(\mathrm{~s}, 3 \mathrm{H})$.

${ }^{13} \mathrm{C}$ NMR $\left(100 \mathrm{MHz}, \mathrm{CDCl}_{3}\right) \delta$ ppm: 78.1, 74.1, 38.6, 31.6, 28.1, 27.8, 23.6, 20.9.

\footnotetext{
${ }^{1}$ Barbier, M.; Hügel, M. F. Bull. Soc. Chim. Fr. 1961, 28, 951-954.
} 
IR (ATR) v cm ${ }^{-1}:$ 3400, 2926, 2859, 1460, 1371, 1261, 1194, 1139, 1096, 1030, 970, 951, 930.

HRMS (El+) $\mathrm{m} / \mathrm{z}$ : calculated for $\mathrm{C}_{8} \mathrm{H}_{16} \mathrm{O}_{2}{ }^{+}\left[\mathrm{M}^{+}\right]$: 144.1145 ; found: 144.1144 .

Measure of the enantiomeric excess of 13:

Compound 13 was first converted to its 2-O-benzoyl ester: To a stirred solution of diol $\mathbf{1 3}(8 \mathrm{mg}, 50$ $\mu \mathrm{mol}, 1$ equiv), DMAP (5 $\mu \mathrm{mol}, 0.1$ equiv) and $\mathrm{NEt}_{3}(10 \mu \mathrm{L}, 75 \mu \mathrm{mol}, 1.5$ equiv) in $500 \mu \mathrm{L}$ of dry DCM, was added $\mathrm{BzCl}(9 \mu \mathrm{L}, 75 \mu \mathrm{mol}, 1.5$ equiv). After stirring at room temperature overnight, the reaction was quenched with a saturated solution of $\mathrm{NH}_{4} \mathrm{Cl}(1 \mathrm{~mL})$. The aqueous layer was extracted with DCM $(3 \times 1 \mathrm{~mL})$. The organic layers were combined, washed with brine, dried over $\mathrm{Na}_{2} \mathrm{SO}_{4}$, filtered and concentrated in vacuo. The ester was passed through a pad of silica (PE/EA 9:1) and analyzed by chiral HPLC, by comparison with retention times $\left(t_{R}\right)$ of a racemic sample prepared from the racemic diol:

HPLC analysis [Nacalai Tesque "COSMOSIL CHiRAL 3B" column: silica gel grafted with cellulose tris $3,5-$ dimethylphenylcarbamate), particle size $3 \mu \mathrm{m}, 4.6 \times 250 \mathrm{~mm}$ ]: elution with $\mathrm{MeCN} / \mathrm{H}_{2} \mathrm{O}=40 / 60,1$ $\mathrm{mL} / \mathrm{min}$; temperature: $40^{\circ} \mathrm{C}$; detection at $230 \mathrm{~nm}$; $t_{R}$ (minor enantiomer) $=14.5 \mathrm{~min}, \mathrm{t}_{\mathrm{R}}$ (major enantiomer) $=15.4 \mathrm{~min} ;$ e.r. $=90: 10$ through peak integration.

\section{(2S)-2-Trimethylsilyloxy-2-methylcycloheptan-1-one (15)}

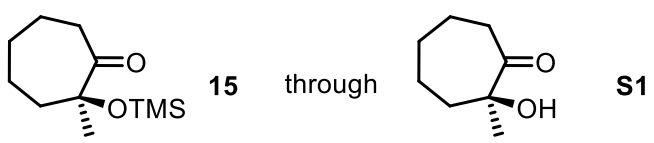

To a stirred solution of $(\mathrm{COCl})_{2}(10 \mathrm{~mL}, 120 \mathrm{mmol}, 1.5$ equiv) in $100 \mathrm{~mL}$ of dry DCM was added a solution of DMSO ( $17 \mathrm{~mL}, 240 \mathrm{mmol}, 3$ equiv) in $100 \mathrm{~mL}$ of dry DCM at $-78{ }^{\circ} \mathrm{C}$ over $1 \mathrm{~h}$. After $10 \mathrm{~min}$, a solution of diol 13 (11.5 g, $80 \mathrm{mmol}, 1$ equiv) in $200 \mathrm{~mL}$ of dry DCM was added dropwise over $1 \mathrm{~h}$. The reaction mixture was stirred for $10 \mathrm{~min}$ then $\mathrm{Et}_{3} \mathrm{~N}$ ( $67 \mathrm{~mL}, 480 \mathrm{mmol}, 6$ equiv) was added over $30 \mathrm{~min}$ and the solution was allowed to warm up slowly to rt. The reaction was quenched with a saturated solution of $\mathrm{NH}_{4} \mathrm{Cl}(300 \mathrm{~mL})$. The aqueous layer was extracted with DCM $(3 \times 400 \mathrm{~mL})$. The organic layers were combined, washed with brine, dried over $\mathrm{Na}_{2} \mathrm{SO}_{4}$, filtered and concentrated in vacuo, to furnish ketone intermediate $\mathbf{S 1}$ as a colorless oil whose NMR data were consistent with those reported in the literature. ${ }^{2}$ Hydroxyketone $\mathbf{S} 1$ was engaged in next step without additional purification.

Data for hydroxyketone S1: ${ }^{1} \mathbf{H}$ NMR $\left(400 \mathrm{MHz}, \mathrm{CDCl}_{3}\right) \delta \mathrm{ppm}$ : $3.84(\mathrm{~s}, 1 \mathrm{H}), 2.75-2.67(\mathrm{~m}, 1 \mathrm{H}), 2.51-2.44$ $(\mathrm{m}, 1 \mathrm{H}), 2.06-1.98(\mathrm{~m}, 1 \mathrm{H}), 1.98-1.93(\mathrm{~m}, 1 \mathrm{H}), 1.86-1.78(\mathrm{~m}, 1 \mathrm{H}), 1.78-1.70(\mathrm{~m}, 1 \mathrm{H}), 1.68-1.62(\mathrm{~m}, 1 \mathrm{H})$, 1.50-1.33 (m, 2H), $1.30(\mathrm{~s}, 3 \mathrm{H}), 1.28-1.17(\mathrm{~m}, 1 \mathrm{H}) .{ }^{13} \mathrm{C} \mathrm{NMR}\left(100 \mathrm{MHz}, \mathrm{CDCl}_{3}\right) \delta \mathrm{ppm}: 216.4,78.7,38.5$, $37.9,30.3,27.2,27.1,23.8$.

To a stirred solution of hydroxy ketone $\mathbf{S 1}(80 \mathrm{mmol}, 1$ equiv) in $150 \mathrm{~mL}$ of DCM was added $\mathrm{N}$ trimethylsilylimidazole ( $17.5 \mathrm{~mL}, 120 \mathrm{mmol}, 1.5$ equiv). After stirring at rt overnight, the reaction was quenched with a saturated solution of $\mathrm{NH}_{4} \mathrm{Cl}(200 \mathrm{~mL}$ ). The aqueous layer was extracted with DCM (3 $x 100 \mathrm{~mL}$ ). The organic layers were combined, washed with brine, dried over $\mathrm{Na}_{2} \mathrm{SO}_{4}$, filtered and concentrated in vacuo. The crude product was purified via silica gel chromatography $\left(\mathrm{PE} / \mathrm{Et}_{2} \mathrm{O}\right.$ 98:2 to $95: 5$ ) to yield $14.4 \mathrm{~g}$ ( $84 \%$ yield over 2 steps) of ketone 15 as a colorless oil.

$[\alpha]_{\mathrm{D}}^{25}=+2.8\left(\mathrm{c} 1.5, \mathrm{CHCl}_{3}\right)$.

\footnotetext{
${ }^{2}$ Blackburn, C.; Childs, R. F.; Kennedy, R. A., Can. J. Chem. 1983, 61, 1981-1986.
} 
${ }^{1} \mathbf{H}$ NMR $\left(400 \mathrm{MHz}, \mathrm{CDCl}_{3}\right) \delta$ ppm: 2.78-2.68 (m, $\left.1 \mathrm{H}\right), 2.40-2.31(\mathrm{~m}, 1 \mathrm{H}), 1.84-1.77(\mathrm{~m}, 1 \mathrm{H}), 1.76-1.68$ $(\mathrm{m}, 3 \mathrm{H}), 1.58-1.48(\mathrm{~m}, 3 \mathrm{H}), 1.33(\mathrm{~s}, 3 \mathrm{H}), 1.30-1.22(\mathrm{~m}, 1 \mathrm{H}), 0.12(\mathrm{~s}, 9 \mathrm{H})$.

${ }^{13} \mathrm{C}$ NMR $\left(100 \mathrm{MHz}, \mathrm{CDCl}_{3}\right) \delta$ ppm: 214.9, 82.3, 40.9, 39.9, 28.9, 26.3, 24.8, 24.7, 2.4.

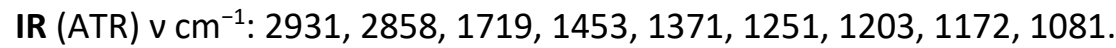

HRMS (EI+) $m / z$ : calculated for $\mathrm{C}_{11} \mathrm{H}_{22} \mathrm{O}_{2} \mathrm{Si}^{+{ }^{+}}\left[\mathrm{M}^{+}\right]$: 214.1384; found: 214.1392 .

(7S)-7-Hydroxy-7-methyl-1-trifluoromethanesulfonyloxycyclohept-1-ene (12)<smiles>OC1=CCCCCC1(O)O</smiles>

To a stirred solution of ketone 15 (5.2 g, 24 mmol, 1 equiv) and PhNTf 2 (12.8 g, 36 mmol, 1.5 equiv) in $200 \mathrm{~mL}$ of dry THF was added a $1 \mathrm{M}$ LiHMDS solution in THF (48 mL, $48 \mathrm{mmol}, 2$ equiv) dropwise over $2 \mathrm{~h}$ at $-78{ }^{\circ} \mathrm{C}$. After stirring at $-78{ }^{\circ} \mathrm{C}$ for $1 \mathrm{~h}$, the reaction mixture was warmed up to $\mathrm{rt}$ and stirred overnight. The reaction was quenched with a solution of $2 \mathrm{M} \mathrm{HCl}(200 \mathrm{~mL})$ at $0{ }^{\circ} \mathrm{C}$ and then stirred at $\mathrm{rt}$ for $1 \mathrm{~h}$. The aqueous layer was extracted with EtOAc $(3 \times 200 \mathrm{~mL})$. The organic layers were combined, washed with a saturated solution of $\mathrm{NaHCO}_{3}$, brine, dried over $\mathrm{Na}_{2} \mathrm{SO}_{4}$, filtered and concentrated in vacuo. The crude product was purified via silica gel chromatography (PE/EA 98:2 to 95:5) to yield $5.7 \mathrm{~g}$ (86\% yield) of enol triflate $\mathbf{1 2}$ as a yellowish oil.

$[\alpha]_{\mathrm{D}}{ }^{25}=+0.9\left(\mathrm{c} 0.9, \mathrm{CHCl}_{3}\right)$.

${ }^{1} \mathrm{H}$ NMR $\left(400 \mathrm{MHz}, \mathrm{CDCl}_{3}\right) \delta$ ppm: $5.86(\mathrm{t}, \mathrm{J}=6.5 \mathrm{~Hz}, 1 \mathrm{H}), 2.29-2.11(\mathrm{~m}, 2 \mathrm{H}), 1.94-1.90(\mathrm{~m}, 2 \mathrm{H}), 1.90-$ $1.81(\mathrm{~m}, 1 \mathrm{H}), 1.73-1.62(\mathrm{~m}, 3 \mathrm{H}), 1.42(\mathrm{~s}, 3 \mathrm{H})$.

${ }^{13} \mathrm{C}$ NMR $\left(100 \mathrm{MHz}, \mathrm{CDCl}_{3}\right) \delta$ ppm: 154.8, 122.7, 120.0 (q, $\left.J=319.5 \mathrm{~Hz}\right), 73.7,39.7,26.2,25.6,23.4$, 22.3.

IR (ATR) v cm ${ }^{-1}: 2941,1411,1247,1204,1143,1003,980$.

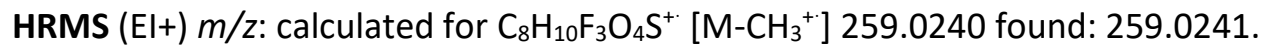

(7S)-7-Acetoxy-7-methyl-1-trifluoromethanesulfonyloxycyclohept-1-ene (16)<smiles>C[C@@]1(C(=O)O)CCCCC=C1[O+]</smiles>

To a stirred solution of enol triflate 12 ( $1.4 \mathrm{~g}, 5 \mathrm{mmol}, 1$ equiv) and isopropenyl acetate $(2.8 \mathrm{~mL}, 25$ mmol, 5 equiv) in $50 \mathrm{~mL}$ of DCM was added TsOH ( $125 \mathrm{mg}, 0.25 \mathrm{mmol}, 0.05$ equiv) at rt. After stirring at $\mathrm{rt}$ overnight, the reaction was quenched with a saturated solution of $\mathrm{NaHCO}_{3}(50 \mathrm{~mL})$. The aqueous layer was extracted with DCM $(3 \times 25 \mathrm{~mL})$. The organic layers were combined, washed with brine, dried over $\mathrm{Na}_{2} \mathrm{SO}_{4}$, filtered and concentrated in vacuo. The crude product was purified via silica gel chromatography (PE/EA 98:2 to 95:5) to yield $1.5 \mathrm{~g}$ (95\% yield) of ester 16 as a colorless oil.

$[\alpha]_{\mathrm{D}}^{25}=-10.5\left(\mathrm{c} 1.1, \mathrm{CHCl}_{3}\right)$.

${ }^{1} \mathrm{H}$ NMR $\left(400 \mathrm{MHz}, \mathrm{CDCl}_{3}\right) \delta \mathrm{ppm}: 5.97(\mathrm{dd}, J=7.6,6.2 \mathrm{~Hz}, 1 \mathrm{H}), 2.49-2.41(\mathrm{~m}, 1 \mathrm{H}), 2.34-2.24(\mathrm{~m}, 1 \mathrm{H})$, 2.21-2.09 (m, 1H), $2.04(\mathrm{~s}, 3 \mathrm{H}), 1.79-1.64(\mathrm{~m}, 3 \mathrm{H}), 1.62(\mathrm{~s}, 3 \mathrm{H})$. 
${ }^{13} \mathrm{C}$ NMR $\left(100 \mathrm{MHz}, \mathrm{CDCl}_{3}\right) \delta$ ppm: 169.8, 151.2, 124.4, 118.5 (q, J = 319.5 Hz), 82.5, 35.0, 25.4, 24.2, 23.5, 23.0, 21.9.

IR (ATR) v cm ${ }^{-1}:$ 2941, 2865, 1744, 1669, 1413, 1369, 1245, 1209, 1142, 1099, 1050, 1009, 985.

HRMS (El+) $m / z$ : calculated for $\mathrm{C}_{10} \mathrm{H}_{15} \mathrm{O}^{+\cdot}\left[\mathrm{M}-\mathrm{OTf}^{+}\right]$]: 167.1062 found: 167.1067 .

(7S)-7-Acetoxy-7-methyl-1-[(1E,3E)-3-methylpenta-1,3-dien-1-yl]cyclohept-1-ene (10), as a 4:1 mixture with the $1 Z$ isomer

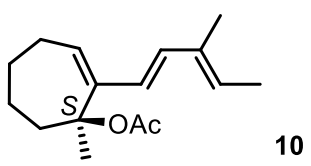

$\mathrm{Pd}(\mathrm{OAc})_{2}$ (113 mg, $0.5 \mathrm{mmol}, 0.1$ equiv.) was dissolved in $35 \mathrm{~mL}$ of dioxane, then $15 \mathrm{~mL}$ of a $2 \mathrm{M}$ solution of $\mathrm{K}_{3} \mathrm{PO}_{4}\left(9.5 \mathrm{~g}, 45 \mathrm{mmmol}, 9\right.$ equiv.) in water were added. The solution was cooled down to $4{ }^{\circ} \mathrm{C}$. After $5 \mathrm{~min}$ at this temperature, enol triflate 16 ( $1.58 \mathrm{~g}, 5 \mathrm{mmol}, 1$ equiv.) was added, followed by pinacol boronate 17 ( $1.15 \mathrm{~g}, 5.5 \mathrm{mmol}, 1.1$ equiv.). After stirring at $4{ }^{\circ} \mathrm{C}$ for $1 \mathrm{~h}$, the reaction was quenched with a saturated solution of $\mathrm{NH}_{4} \mathrm{Cl}(50 \mathrm{~mL})$. The aqueous layer was extracted with pentane $(3 \times 50 \mathrm{~mL})$. The organic layers were combined, washed with brine, dried over $\mathrm{Na}_{2} \mathrm{SO}_{4}$, filtered and concentrated in vacuo. The crude product was purified via silica gel chromatography (pentane/Et ${ }_{2} \mathrm{O} 98: 2$ ) to yield 880 $\mathrm{mg}(71 \%$ yield) of triene 10 as a colorless oil.

$[\alpha]_{D}^{25}=+29.1\left(\mathrm{c} 0.8, \mathrm{CHCl}_{3}\right)$.

${ }^{1} \mathrm{H}$ NMR $\left(400 \mathrm{MHz}, \mathrm{CDCl}_{3}\right) \delta \mathrm{ppm}: 6.35(\mathrm{~d}, J=15.9 \mathrm{~Hz}, 1 \mathrm{H}), 6.08$ (d, $\left.J=15.9 \mathrm{~Hz}, 1 \mathrm{H}\right), 5.93$ (ddd, $J=7.9$, 5.3, 1.0 Hz, 1H), 5.58-5.50 (m, 1H), 2.75-2.65 (m, 1H), 2.39-2.29 (m, 1H), 2.18-2.10 (m, $1 \mathrm{H}), 1.97(\mathrm{~s}, 3 \mathrm{H})$, $1.88-1.80(\mathrm{~m}, 1 \mathrm{H}), 1.79-1.75(\mathrm{~m}, 1 \mathrm{H}), 1.74(\mathrm{~s}, 3 \mathrm{H}), 1.72(\mathrm{~s}, 3 \mathrm{H}), 1.71-1.65(\mathrm{~m}, 2 \mathrm{H}), 1.62-1.57(\mathrm{~m}, 1 \mathrm{H})$, $1.53(\mathrm{~s}, 3 \mathrm{H})$.

${ }^{13} \mathrm{C}$ NMR $\left(100 \mathrm{MHz}, \mathrm{CDCl}_{3}\right) \delta$ ppm: 169.9, 144.4, 135.0, 133.6, 128.3, 126.5, 126.5, 85.5, 36.6, 27.0, 26.0, 25.9, 23.6, 22.2, 14.1, 12.1 .

IR (ATR) v cm${ }^{-1}:$ 2928, 2858, 1737, 1445, 1367, 1244, 1166, 1144, 1081, 1014, 959.

HRMS (El+) $\mathrm{m} / \mathrm{z}$ : calculated for $\mathrm{C}_{16} \mathrm{H}_{24} \mathrm{O}_{2}{ }^{+\cdot}\left[\mathrm{M}^{+}\right]$: 248.1771; found: 248.1777 .

Control of the enantiomeric ratio:

HPLC analysis [Nacalai Tesque "COSMOSIL CHIRAL 3B" column: silica gel grafted with cellulose tris(3,5dimethylphenylcarbamate), particle size $3 \mu \mathrm{m}, 4.6 \times 250 \mathrm{~mm}$ ]: elution with $\mathrm{MeCN} / \mathrm{H}_{2} \mathrm{O}=90 / 10,0.6$ $\mathrm{mL} / \mathrm{min}$; temperature: $40^{\circ} \mathrm{C}$; detection at $230 \mathrm{~nm}$; $t_{R}$ (minor enantiomer) $=19.6 \mathrm{~min}, \mathrm{t}_{\mathrm{R}}$ (major enantiomer) $=20.4 \mathrm{~min}$; e.r. $=90: 10$ through peak integration .

(rac)-2-[3-Methyl-2-vinylcyclohept-2-en-1-yl]acetic acid (S2)<smiles>C=CC1=C(C)CCCCC1CC(=O)O</smiles> 
To a solution of 1-methyl-2-vinylcyclohept-2-en-1-yl acetate $18^{3}$ (564 mg, $2.90 \mathrm{mmol}$ ) in toluene (10 $\mathrm{mL}$ ) at $-78{ }^{\circ} \mathrm{C}$ was added dropwise LiHMDS (1M in THF, $3.48 \mathrm{~mL}, 3.48 \mathrm{mmol}$ ). After stirring at this temperature for $30 \mathrm{~min}$, a solution of freshly distilled $\mathrm{TMSCl}(0.59 \mathrm{~mL}, 4.64 \mathrm{mmol})$ and $\mathrm{Et}_{3} \mathrm{~N}(0.65 \mathrm{~mL}$, $4.64 \mathrm{mmol})$ in toluene $(10 \mathrm{~mL}$ ) was added dropwise. After stirring for $30 \mathrm{~min}$, the cooling bath was removed and the reaction mixture was stirred at $\mathrm{rt}$ for $1 \mathrm{~h}$, before being refluxed overnight. After cooling the mixture was poured into an aqueous solution of $\mathrm{NaOH}(2 \mathrm{M}, 11 \mathrm{~mL}, 22 \mathrm{mmol})$ and stirred for $15 \mathrm{~min}$, followed by dilution with Et2O $(10 \mathrm{~mL})$ and extraction with aqueous $\mathrm{NaOH}(3 \times 20 \mathrm{~mL}, 2 \mathrm{M})$. The combined aqueous phases were acidified to $\mathrm{pH} 3$ with hydrochloric acid $(2 \mathrm{M})$ and extracted with $\mathrm{Et}_{2} \mathrm{O}(3 \times 20 \mathrm{~mL})$. Drying $\left(\mathrm{MgSO}_{4}\right)$ and evaporation of the solvent afforded acid $\mathbf{S 2}$ as a pale yellow oil (335 mg, 59\%). The crude material was taken on without further purification.

${ }^{1} \mathrm{H}$ NMR $\left(400 \mathrm{MHz}, \mathrm{CDCl}_{3}\right) \delta$ ppm: $6.59(1 \mathrm{H}, \mathrm{dd}, J=17.2,11.1 \mathrm{~Hz}), 5.20(1 \mathrm{H}, \mathrm{d}, J=17.2 \mathrm{~Hz}), 5.00(1 \mathrm{H}, \mathrm{d}$, $J=11.1 \mathrm{~Hz}), 3.23-3.33(1 \mathrm{H}, \mathrm{m}), 2.49-2.65(2 \mathrm{H}, \mathrm{m}), 2.31-2.43(1 \mathrm{H}, \mathrm{m}), 2.04-2.17(1 \mathrm{H}, \mathrm{m}), 1.83(3 \mathrm{H}, \mathrm{s})$, 1.59-1.79 $(5 \mathrm{H}, \mathrm{m}), 1.39-1.51(1 \mathrm{H}, \mathrm{m})$.

${ }^{13} \mathrm{C}$ NMR $\left(100 \mathrm{MHz}, \mathrm{CDCl}_{3}\right) \delta \mathrm{ppm}: 179.5,138.6,136.0,134.8,111.6,36.7,35.3,35.1,29.3,25.9,25.0$, 22.7.

IR (film on NaCl) v cm${ }^{-1}: 3055,2986,1420,1265,895,737,706$.

HRMS (ESI+) $m / z$ : calculated for $\mathrm{C}_{12} \mathrm{H}_{19} \mathrm{O}_{2}{ }^{+}\left[\mathrm{MH}^{+}\right]$: 195.1385 ; found: 195.1383 .

(rac)-2-[3-Methyl-2-vinylcyclohept-2-en-1-yl]acetic acid, methyl ester (19)<smiles>C=CC1=C(C)CCCCC1CC(C)=O</smiles>

To a stirred solution of $\mathbf{S 2}(330 \mathrm{mg}, 0.31 \mathrm{mmol})$ in toluene/methanol $(3.6: 1 ; 17 \mathrm{~mL})$ at $0{ }^{\circ} \mathrm{C}$ was added dropwise $\mathrm{TMSCHN}_{2}(2.0 \mathrm{M}$ in Et $2 \mathrm{O}, 0.94 \mathrm{~mL}, 1.87 \mathrm{mmol})$. The cooled bath was removed and the mixture was stirred for 30 min before being concentrated. The crude material was purified by chromatography on silica gel (100:3 cyclohexane/EtOAc) to afford ester 19 as a colorless oil (317 mg, 91\%).

${ }^{1} \mathrm{H}$ NMR $\left(400 \mathrm{MHz}, \mathrm{CDCl}_{3}\right) \delta \mathrm{ppm:} 6.57(1 \mathrm{H}, \mathrm{dd}, J=11.1,17.2 \mathrm{~Hz}), 5.19(1 \mathrm{H}, \mathrm{d}, J=17.2 \mathrm{~Hz}), 5.00(1 \mathrm{H}, \mathrm{d}$, $J=11.1 \mathrm{~Hz}), 3.66(3 \mathrm{H}, \mathrm{s}), 3.23-3.32(1 \mathrm{H}, \mathrm{m}), 2.45-2.61(2 \mathrm{H}, \mathrm{m}), 2.31-2.44(1 \mathrm{H}, \mathrm{m}), 2.02-2.14(1 \mathrm{H}, \mathrm{m})$, $1.82(3 \mathrm{H}, \mathrm{s}), 1.58-1.76(5 \mathrm{H}, \mathrm{m}), 1.37-1.51(1 \mathrm{H}, \mathrm{m})$.

${ }^{13} \mathrm{C}$ NMR $\left(100 \mathrm{MHz}, \mathrm{CDCl}_{3}\right) \delta$ ppm: 173.6, 138.3, 136.0, 134.9, 111.3, 51.4, 36.5, 35.2, 35.1, 29.4, 25.7, 24.9, 22.6 .

IR (film on NaCl) v cm-1: 3055, 2986, 2928, 1732, 1424, 1265, 895.

HRMS (ESI+) $\mathrm{m} / z$ : calculated for $\mathrm{C}_{13} \mathrm{H}_{21} \mathrm{O}_{2}{ }^{+}\left[\mathrm{MH}^{+}\right]$: 209.1536; found: 209.1539 .

(E)-2-(3-Methyl-2-(2-(4,4,5,5-tetramethyl-1,3,2-dioxaborolan-2-yl)vinyl)cyclohept-2-en-1-yl)acetic acid, methyl ester (20)

\footnotetext{
${ }^{3}$ Laroche, B.; Detraz, M.; Blond, A.; Dubost, L.; Mailliet, P.; Nay, B. J. Org. Chem. 2015, 80, 5359-5363.
} 


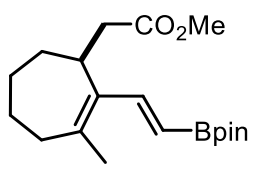

To a flame-dried round bottom flask was added second generation Hoveyda-Grubbs catalyst $(84 \mathrm{mg}$, $0.13 \mathrm{mmol})$, followed by compound $19(278 \mathrm{mg}, 1.34 \mathrm{mmol})$ in distilled toluene $(13 \mathrm{~mL})$ and pinacol boronate $(0.91 \mathrm{~mL}, 5.35 \mathrm{mmol})$. The reaction mixture was stirred at $80^{\circ} \mathrm{C}$ for $2 \mathrm{~h}$, and a second portion of the Hoveyda-Grubbs catalyst was added ( $84 \mathrm{mg}, 0.13 \mathrm{mmol}$ ). After 2 extra hours at $80^{\circ} \mathrm{C}$ and entire consumption of the starting material, the reaction mixture was cooled down to rt and concentrated. The resulting crude material was purified by chromatography on silica gel $\left(100 \% \mathrm{CH}_{2} \mathrm{Cl}_{2}\right)$ to afford pinacol boronate $\mathbf{2 0}$ as a pale yellow oil (320 mg, 72\%).

${ }^{1} \mathrm{H}$ NMR $\left(400 \mathrm{MHz}, \mathrm{CDCl}_{3}\right) \delta$ ppm: $7.37(1 \mathrm{H}, \mathrm{d}, J=18.3 \mathrm{~Hz}), 5.57(1 \mathrm{H}, \mathrm{d}, J=18.3 \mathrm{~Hz}), 3.64(3 \mathrm{H}, \mathrm{s}), 3.32-$ $3.42(1 \mathrm{H}, \mathrm{m}), 2.53-2.63(1 \mathrm{H}, \mathrm{m}), 2.37-2.49(2 \mathrm{H}, \mathrm{m}), 2.02-2.13(1 \mathrm{H}, \mathrm{m}), 1.92(3 \mathrm{H}, \mathrm{s}), 1.54-1.78(5 \mathrm{H}, \mathrm{m})$, $1.34-1.46(1 \mathrm{H}, \mathrm{m}), 1.27(12 \mathrm{H}, \mathrm{s})$.

${ }^{13} \mathrm{C} \mathrm{NMR}$ ( $75 \mathrm{MHz}, \mathrm{CDCl}_{3}$ ) $\delta \mathrm{ppm}$ (the carbon bearing boron could not be observed): 173.3, 148.7, 142.8, $135.9,83.0,51.4,36.4,35.5,34.6,29.0,25.6,24.8,24.7,24.5,22.9$.

IR (film on NaCl) v cm${ }^{-1}: 3042,2978,2928,2857,1732,1615,1593,1379,1371,1343,1321,1146$.

HRMS (ESI+) $\mathrm{m} / z$ : calculated for $\mathrm{C}_{9} \mathrm{H}_{32} \mathrm{BO}_{4}{ }^{+}[\mathrm{MH}]^{+}:$335.2394; found: 335.2404 .

\section{(E)-2-lodobut-2-ene (21)}

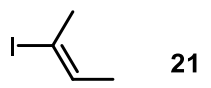

Following Klapars and Buchwald procedure, ${ }^{4}$ a Schlenk tube was charged with Cul $(95 \mathrm{mg}, 0.50 \mathrm{mmol}$, $5.0 \mathrm{~mol} \%)$, Nal $(2.25 \mathrm{~g}, 15.0 \mathrm{mmol})$, evacuated and backfilled with argon. $N, N^{\prime}-$ Dimethylethylenediamine $(0.11 \mathrm{~mL}, 1.00 \mathrm{mmol}, 10 \mathrm{~mol} \%)$, commercial $(E)$-2-bromobut-2-ene (1.01 $\mathrm{mL}, 10.0 \mathrm{mmol})$, and $n$-butanol $(5 \mathrm{~mL})$ were added under argon. The Schlenk tube was sealed and the reaction mixture was stirred at $120^{\circ} \mathrm{C}$ for $24 \mathrm{~h}$. The resulting suspension was allowed to reach room temperature, poured into pentane $(50 \mathrm{~mL}$ ) and washed with a solution of $20 \%$ aqueous ammonia ( 3 $\mathrm{mL})$ in water $(50 \mathrm{~mL})$, followed by water $(3 \times 50 \mathrm{~mL})$. The organic phase was dried $\left(\mathrm{MgSO}_{4}\right)$ and concentrated to $\sim 2 \mathrm{~mL}$ volume. The residue was distilled collecting the fraction boiling at $116-122{ }^{\circ} \mathrm{C}$ to give $1.155 \mathrm{~g}$ (63\% yield) of $\mathbf{2 1}$ as a colorless liquid. Data were accordance with Shu and Djerassi's previous report. ${ }^{5}$

${ }^{1} \mathrm{H}$ NMR $\left(400 \mathrm{MHz}, \mathrm{CDCl}_{3}\right) \delta \mathrm{ppm}: 6.21(1 \mathrm{H}, \mathrm{qq}, J=1.2,6.9 \mathrm{~Hz}), 2.36(3 \mathrm{H}$, pseudo-quint, $J=1.2 \mathrm{~Hz}), 1.61$ $(3 \mathrm{H}, \mathrm{dq}, J=1.2,6.9 \mathrm{~Hz})$.

${ }^{13} \mathrm{C}$ NMR $\left(75 \mathrm{MHz}, \mathrm{CDCl}_{3}\right) \delta$ ppm: 135.5, 93.8, 27.0, 16.0,

IR (film on NaCl) v cm${ }^{-1}$ : 3036, 2970, 2947, 2916, 2855, 1640, 1597, 1427, 1377, 1115, 1050, 995, 814.

\footnotetext{
${ }^{4}$ Klapars, A.; Buchwald, S.L. J. Am. Chem. Soc. 2002, 124, 14844-14845.

${ }^{5}$ Shu, A.Y.L.; Djerassi, C. J. Chem. Soc. Perkin Trans. 1 1987, 1291-1305.
} 
2-(3-Methyl-2-((1E,3E)-3-methylpenta-1,3-dien-1-yl)cyclohept-2-en-1-yl)acetic acid, methyl ester (S3)

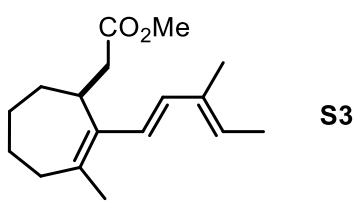

$\mathrm{Pd}(\mathrm{OAc})_{2}(21 \mathrm{mg}, 0.093 \mathrm{mmol})$ and XPhos $(89 \mathrm{mg}, 0.19 \mathrm{mmol})$ were added in a round bottom flask under argon, and stirred in dry THF $(7 \mathrm{~mL})$ at $\mathrm{rt}$ for $30 \mathrm{~min}$ (brown solution). Then (E)-2-iodobut-2-ene 21 (187 mg, $1.03 \mathrm{mmol}$ ) in THF (1 mL), boronate 20 (312 mg, $0.93 \mathrm{mmol}$ ) in THF (1 mL) and an aqueous solution of $\mathrm{NaOH}(2 \mathrm{M}, 2.3 \mathrm{~mL}, 4.67 \mathrm{mmol})$ were added, and the mixture was stirred for $16 \mathrm{~h}$ at $\mathrm{rt}$ in the dark. The reaction was quenched using a saturated solution of $\mathrm{NH}_{4} \mathrm{Cl}(8 \mathrm{~mL})$, extracted with $\mathrm{Et}_{2} \mathrm{O}(2 \mathrm{x}$ $10 \mathrm{~mL}$ ), dried over $\mathrm{Na}_{2} \mathrm{SO}_{4}$ and concentrated under reduced pressure. The resulting crude material was purified by chromatography on silica gel (100:3 cyclohexane/EtOAc) to afford triene S3 as an orange oil (188 mg, 77\%).

${ }^{1} \mathrm{H}$ NMR $\left(400 \mathrm{MHz}, \mathrm{CDCl}_{3}\right) \delta \mathrm{ppm}: 6.34(1 \mathrm{H}, \mathrm{d}, J=16.0 \mathrm{~Hz}), 6.28(1 \mathrm{H}, \mathrm{d}, J=16.0 \mathrm{~Hz}), 5.59(1 \mathrm{H}, \mathrm{q}, J=7.0$, $14.0 \mathrm{~Hz}), 3.66(3 \mathrm{H}, \mathrm{s}), 3.28-3.34(1 \mathrm{H}, \mathrm{m}), 2.59(1 \mathrm{H}, \mathrm{d}, J=10.1,14.9 \mathrm{~Hz}), 2.50(1 \mathrm{H}, \mathrm{dd}, J=5.6,14.9 \mathrm{~Hz})$, 2.36-2.44 $(1 \mathrm{H}, \mathrm{m}), 2.09-2.15(1 \mathrm{H}, \mathrm{m}), 1.86(3 \mathrm{H}, \mathrm{s}), 1.80(3 \mathrm{H}, \mathrm{s}), 1.76(3 \mathrm{H}, \mathrm{d}, J=7.0 \mathrm{~Hz}), 1.69-1.74(1 \mathrm{H}$, m), 1.57-1.70 (4H, m, 9-H, 10-Hb), 1.40-1.48 (1H, m).

${ }^{13} \mathrm{C} \mathrm{NMR}\left(75 \mathrm{MHz}, \mathrm{CDCl}_{3}\right) \delta$ ppm: 173.7, 137.1, 135.2, 134.9, 131.7, 126.3, 125.4, 51.5, 36.8, 36.0, 35.3, 29.6, 26.0, 25.1, 22.8, 14.0, 12.1 .

IR (film on NaCl) v cm${ }^{-1}: 3061,2988,2922,2855,1738,1435,1287,1265,1244,1167,1138$.

HRMS (ESI+) $m / z$ : calculated for $\mathrm{C}_{17} \mathrm{H}_{27} \mathrm{O}_{2}{ }^{+}\left[\mathrm{MH}^{+}\right]: 263.2006$; found: 263.2004 .

(rac)-2-[3-Methyl-2-((1E,3E)-3-methylpenta-1,3-dien-1-yl)cyclohept-2-en-1-yl]acetic acid (rac-9)

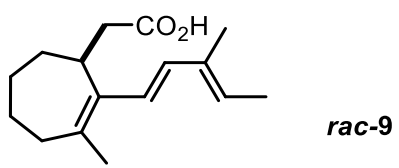

An argon-bubbled aqueous solution of $\mathrm{NaOH}(14.8 \mathrm{M}, 1.02 \mathrm{~mL}, 15.1 \mathrm{mmol})$ was added to a solution of ester $\mathbf{S 3}$ (198 $\mathrm{mg}, 0.76 \mathrm{mmol}$ ) in EtOH also previously argon-bubbled $(8 \mathrm{~mL})$. The reaction mixture was stirred at $\mathrm{rt}$ for $7 \mathrm{~h}$ in the dark before being quenched by an aqueous solution of tartaric acid $(0.73 \mathrm{M}$, $20 \mathrm{~mL}, 14.8 \mathrm{mmol}$ ) and extracted with $\mathrm{Et}_{2} \mathrm{O}(4 \times 10 \mathrm{~mL})$. The combined organic layers were dried over $\mathrm{Na}_{2} \mathrm{SO}_{4}$ and concentrated under reduced pressure to afford rac-9 as an yellowish oil that was used without further purification (180 mg, 96\%). Data were

${ }^{1} \mathrm{H}$ NMR (400 MHz, CDCl $)_{3} \delta$ ppm: $6.33(\mathrm{~d}, \mathrm{~J}=16.0 \mathrm{~Hz}, 1 \mathrm{H}), 6.27(\mathrm{~d}, \mathrm{~J}=16.0 \mathrm{~Hz}, 1 \mathrm{H}), 5.58(\mathrm{q}, \mathrm{J}=14.0$, 7.0, 1H), 3.35-3.24 (m, 1H), $2.62(\mathrm{dd}, \mathrm{J}=15.2,10.4,1 \mathrm{H}), 2.51(\mathrm{dd}, \mathrm{J}=15.2,5.0 \mathrm{~Hz}, 1 \mathrm{H}), 2.43-2.31(\mathrm{~m}$, $1 \mathrm{H}), 2.16-2.06(\mathrm{~m}, 1 \mathrm{H}), 1.85(\mathrm{~s}, 3 \mathrm{H}), 1.78(\mathrm{~s}, 3 \mathrm{H}), 1.74(\mathrm{~d}, \mathrm{~J}=7.0 \mathrm{~Hz}, 3 \mathrm{H}), 1.72-1.58(\mathrm{~m}, 5 \mathrm{H}), 1.51-1.38$ $(\mathrm{m}, 1 \mathrm{H})$.

${ }^{13} \mathrm{C}$ NMR $\left(100 \mathrm{MHz}, \mathrm{CDCl}_{3}\right) \delta$ ppm: 179.7, 137.1, 135.1, 134.7, 131.8, 126.4, 125.2, 36.7, 35.8, 35.2, $29.3,25.8,24.9,22.9,14.0,12.1$.

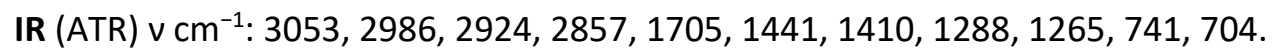


HRMS (El+) $m / z$ : calculated for $\mathrm{C}_{16} \mathrm{H}_{24} \mathrm{O}_{2}^{+\cdot}\left[\mathrm{M}^{+}\right]$: 248.1771; found: 248.1772 .

2-[(1S)-3-Methyl-2-((1E,3E)-3-methylpenta-1,3-dien-1-yl)cyclohept-2-en-1-yl]acetic acid (9)

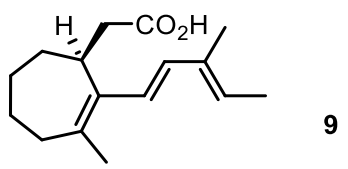

To a stirred solution of triene 10 ( $860 \mathrm{mg}, 3.5 \mathrm{mmol}, 1$ equiv) and TPPA ( $1.6 \mathrm{~mL}, 7 \mathrm{mmol}, 2$ equiv) in 20 $\mathrm{mL}$ of dry THF was added a $1 \mathrm{M}$ LDA solution in THF/hexane $(7 \mathrm{~mL}, 7 \mathrm{mmol}, 2$ equiv) dropwise over 20 min at $-78{ }^{\circ} \mathrm{C}$. After $1 \mathrm{~h}$, a solution of TBSCl $(1.05 \mathrm{~g}, 7 \mathrm{mmol}, 2$ equiv) in $7 \mathrm{~mL}$ of dry THF was added dropwise over $20 \mathrm{~min}$. After stirring at $-78^{\circ} \mathrm{C}$ for $1 \mathrm{~h}$, the reaction mixture was warmed up to $\mathrm{rt}$ and stirred overnight. The reaction mixture was cooled down to $0{ }^{\circ} \mathrm{C}$ and a $1 \mathrm{M}$ solution of TBAF in THF (10.5 mL, $10.5 \mathrm{mmol}, 3$ equiv) was added. The reaction mixture was warmed up to rt, stirred for $1 \mathrm{~h}$ and $150 \mathrm{~mL}$ of pentane were added. The slurry was filtered and washed with pentane. The precipitate was dissolved with a saturated solution $\mathrm{NH}_{4} \mathrm{Cl}$ and extracted with $\mathrm{Et}_{2} \mathrm{O}(3 \times 50 \mathrm{~mL})$. The organic layers were combined, washed with brine, dried over $\mathrm{Na}_{2} \mathrm{SO}_{4}$, filtered and concentrated in vacuo to yield 617 $\mathrm{mg}$ ( $71 \%$ yield) of crude carboxylic acid $\mathbf{9}$ which was engaged in the next step without additional purification.

Data were consistent with those obtained for rac-9.

$[\alpha]_{\mathrm{D}}^{25}=+29.1\left(\mathrm{c} 0.8, \mathrm{CHCl}_{3}\right)$.

N-Benzoyl-5-isobutyl-3-\{2-[(1S)-3-methyl-2-((1E,3E)-3-methylpenta-1,3-dien-1-yl)cyclohept-2-en-1yl]acetyl\}-2-pyrrolidone (mixture of stereoisomers 22)

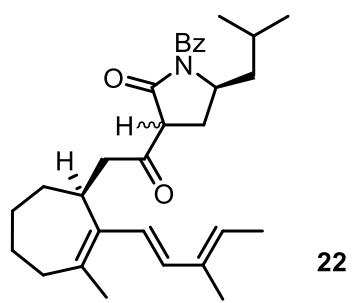

To a stirred solution of carboxylic acid 9 (62 $\mathrm{mg}, 0.25 \mathrm{mmol}, 1$ equiv) in $2 \mathrm{~mL}$ of dry DCM was added $\mathrm{Et}_{3} \mathrm{~N}$ (70 $\mu \mathrm{L}, 0.25 \mathrm{mmol}, 1$ equiv), followed by a solution of pivaloyl chloride ( $60 \mu \mathrm{L}, 0.5 \mathrm{mmol}, 2$ equiv) in $0.5 \mathrm{~mL}$ of DCM dropwise at $-78^{\circ} \mathrm{C}$. The reaction mixture was then warmed up to $0{ }^{\circ} \mathrm{C}$ and stirred in the dark for $30 \mathrm{~min}$ before being quenched with $4 \mathrm{~mL}$ of cold water. The aqueous layer was extracted with $\mathrm{Et}_{2} \mathrm{O}(3 \times 5 \mathrm{~mL})$. The organic layers were combined, washed with cold water, cold brine, dried over $\mathrm{Na}_{2} \mathrm{SO}_{4}$, filtered and concentrated in vacuo to yield a yellowish oil. This resulting mixt anhydride was directly engaged in the next step without additional purification. Short description of this intermediate:

${ }^{1} \mathrm{H}$ NMR $\left(400 \mathrm{MHz}, \mathrm{CDCl}_{3}\right) \delta \mathrm{ppm}: 6.33(\mathrm{~d}, \mathrm{~J}=16.1 \mathrm{~Hz}, 1 \mathrm{H}), 6.24(\mathrm{~d}, \mathrm{~J}=16.1 \mathrm{~Hz}, 1 \mathrm{H}), 5.56(\mathrm{q}, J=13.1$, $6.7 \mathrm{~Hz}, 1 \mathrm{H}), 3.38-3.29(\mathrm{~m}, 1 \mathrm{H}), 3.17(\mathrm{td}, J=6.7,3.4 \mathrm{~Hz}, 1 \mathrm{H}), 2.75-2.59(\mathrm{~m}, 1 \mathrm{H}), 2.42-2.33(\mathrm{~m}, 1 \mathrm{H}), 2.16-$ $2.08(\mathrm{~m}, 1 \mathrm{H}), 1.85(\mathrm{~s}, 3 \mathrm{H}), 1.79(\mathrm{t}, J=1.1 \mathrm{~Hz}, 3 \mathrm{H}), 1.74(\mathrm{brs}, 3 \mathrm{H}), 1.78-1.60(\mathrm{~m}, 5 \mathrm{H}), 1.50-1.39(\mathrm{~m}, 1 \mathrm{H})$, $1.26(\mathrm{~s}, 9 \mathrm{H})$. 
To a stirred solution of $\mathrm{Y}$-lactam $\mathbf{8}^{6}$ ( $120 \mathrm{mg}, 0.5 \mathrm{mmol}, 2$ equiv) in $1 \mathrm{~mL}$ of dry THF was added a $1 \mathrm{M}$ solution of LiHMDS in THF $\left(0.5 \mathrm{~mL}, 0.5 \mathrm{mmol}, 2\right.$ equiv) dropwise at $-78^{\circ} \mathrm{C}$. The reaction mixture was stirred $1 \mathrm{~h}$ at this temperature before adding a cold solution of anhydride 32 ( $0.25 \mathrm{mmol}, 1$ equiv) in 1 $\mathrm{mL}$ of dry THF dropwise. After $5 \mathrm{~h}$ at $-78{ }^{\circ} \mathrm{C}$, the reaction mixture was quenched with a saturated solution of $\mathrm{NH}_{4} \mathrm{Cl}(4 \mathrm{~mL})$ and then allowed to warmed up to $\mathrm{rt}$. The aqueous layer was extracted with $\mathrm{Et}_{2} \mathrm{O}(3 \times 5 \mathrm{~mL})$. The organic layers were combined, washed with brine, dried over $\mathrm{Na}_{2} \mathrm{SO}_{4}$, filtered and concentrated in vacuo. The crude product was purified via silica gel chromatography ( $\left.\mathrm{PE} / \mathrm{Et}_{2} \mathrm{O} 9: 1\right)$ to yield $69 \mathrm{mg}$ ( $58 \%$ yield over the 2 steps from 9 ) of product 22 (mixture of stereoisomers).

Physical state: yellowish oil.

$[\alpha]_{D}^{25}=+56.2\left(\mathrm{c} 0.8, \mathrm{CHCl}_{3}\right)$.

${ }^{1} \mathrm{H}$ NMR $\left(400 \mathrm{MHz}, \mathrm{CDCl}_{3}\right) \delta$ ppm: 7.65-7.48 (m, 3H), 7.45-7.35 (m, 2H), 6.46-6.06 (m, 2H), 5.65-5.46 $(\mathrm{m}, 1 \mathrm{H}), 4.62-4.31(\mathrm{~m}, 1 \mathrm{H}), 3.50-3.22(\mathrm{~m}, 1 \mathrm{H}), 2.60-1.98(\mathrm{~m}, 5 \mathrm{H}), 1.92-1.52(\mathrm{~m}, 17 \mathrm{H}), 1.51-1.27(\mathrm{~m}, 3 \mathrm{H})$, $1.04-0.93(\mathrm{~m}, 6 \mathrm{H})$.

${ }^{13} \mathrm{C}$ NMR $\left(100 \mathrm{MHz}, \mathrm{CDCl}_{3}\right) \delta$ ppm: 204.1, 172.6, 170.6, 136.8, 135.4, 135.1, 134.4, 134.4, 132.1, 131.9, 129.1, 127.9, 126.4, 125.6, 56.0, 54.5, 45.5, 43.3, 34.8, 30.1, 29.9, 27.0, 25.8, 25.4, 23.8, 22.8, 21.6, 14.0, 12.6 .

IR (ATR) v cm${ }^{-1}$ : 3059, 3030, 2957, 2926, 2868, 2855, 1713, 1665, 1632, 1449, 1290, 1281, 1236.

HRMS (ESI+) $\mathrm{m} / \mathrm{z}$ : calculated for $\mathrm{C}_{31} \mathrm{H}_{42} \mathrm{NO}_{3}{ }^{+}\left[\mathrm{MH}^{+}\right]$: 476.3159; found: 476.3159 .

N-Benzoyl-5-isobutyl-3-\{2-[(1S)-3-methyl-2-((1E,3E)-3-methylpenta-1,3-dien-1-yl)cyclohept-2-en-1yl]acetyl\}-3-phenylselenyl-2-pyrrolidone (mixture of stereoisomers 23)

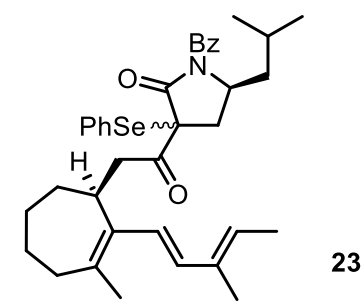

To a stirred solution of triene 22 (48 mg, $109 \mu \mathrm{mol}, 1$ equiv) in $1.5 \mathrm{~mL}$ of dry THF was added a $1 \mathrm{M}$ solution of LiHMDS in THF $\left(120 \mu \mathrm{L}, 120 \mu \mathrm{mol}, 1.1\right.$ equiv) dropwise at $-78^{\circ} \mathrm{C}$. The reaction mixture was stirred $1 \mathrm{~h}$ at this temperature before adding a cold solution of $\mathrm{PhSeBr}$ ( $36 \mathrm{mg}, 150 \mu \mathrm{mol}, 1.4$ equiv) in $0.5 \mathrm{~mL}$ of dry THF dropwise. After $2 \mathrm{~h}$ at $-78^{\circ} \mathrm{C}$, the reaction mixture was quenched with a saturated solution of $\mathrm{NH}_{4} \mathrm{Cl}(2 \mathrm{~mL})$ and then allowed to warmed up to $\mathrm{rt}$. The aqueous layer was extracted with $\mathrm{Et}_{2} \mathrm{O}(3 \times 5 \mathrm{~mL})$. The organic layers were combined, washed with brine, dried over $\mathrm{Na}_{2} \mathrm{SO}_{4}$, filtered and concentrated in vacuo. The crude product was purified via silica gel chromatography ( $\mathrm{PE} / \mathrm{Et}_{2} \mathrm{O} 20: 1$ ) to yield $65 \mathrm{mg}$ (95\% yield) of selenide $\mathbf{2 3}$ as a yellowish oil.

$[\alpha]_{D}^{25}=+109.1\left(\mathrm{c} 1.0, \mathrm{CHCl}_{3}\right)$.

${ }^{1} \mathrm{H}$ NMR (400 MHz, $\left.\mathrm{CDCl}_{3}\right) \delta$ ppm: 7.27-7.68 (m, 10H), 6.42-6.06 (m, 2H), 5.63-5.36 (m, $\left.1 \mathrm{H}\right)$, 4.38-4.06 $(\mathrm{m}, 1 \mathrm{H}), 2.55-1.93(\mathrm{~m}, 3 \mathrm{H}), 1.90-1.46(\mathrm{~m}, 19 \mathrm{H}), 1.38-1.08(\mathrm{~m}, 3 \mathrm{H}), 0.96-0.76(\mathrm{~m}, 6 \mathrm{H})$.

\footnotetext{
${ }^{6}$ Smrcina, M.; Majer, P.; Majerová, E.; Guerassina, T. A.; Eissenstat, M. A. Tetrahedron 1997, 53, $12867-$ 12874.
} 
${ }^{13} \mathrm{C}$ NMR $\left(100 \mathrm{MHz}, \mathrm{CDCl}_{3}\right) \delta$ ppm: 201.3, 200.3, 170.9, 137.6, 137.2, 135.3, 134.5, 132.7, 131.4, 130.2, $129.4,129.3,128.1,125.9,60.7,54.2,53.1,40.5,35.4,32.6,29.7,25.9,25.2,24.8,23.9,22.8,21.2$, 14.1, 12.3 .

IR (ATR) v cm ${ }^{-1}:$ 2957, 2924, 2866, 2857, 1724, 1690, 1288, 1273, 1231.

HRMS (ESI+) m/z: calculated for $\mathrm{C}_{37} \mathrm{H}_{46} \mathrm{NO}_{3} \mathrm{Se}^{+}\left[\mathrm{MH}^{+}\right]$: 632.2637; found: 632.2628 .

(3S,3aR,4S,6aS,8aS,13bR)-5-Benzoyl-4-isobutyl-2,3,13-trimethyl-4,5,8,8a,9,10,11,12-octahydro-3Hcyclohepta[3,4]benzo[1,2-d]isoindole-6,7(3aH,13bH)-dione (endo product 24)

and

(3R,3aR,4S,6aS,8aS,13bS)-5-Benzoyl-4-isobutyl-2,3,13-trimethyl-4,5,8,8a,9,10,11,12-octahydro-3Hcyclohepta[3,4]benzo[1,2-d]isoindole-6,7(3aH,13bH)-dione (exo product 25$)$
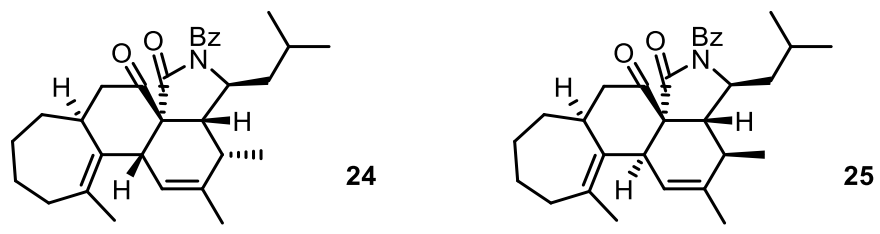

To a stirred solution of selenide 23 (70 mg, $110 \mu$ mol, 1 equiv) in $10 \mathrm{~mL}$ of dry DCM was added $\mathrm{NaHCO}_{3}$ (20 mg, $236 \mu \mathrm{mol}, 2.1$ equiv) at $0{ }^{\circ} \mathrm{C}$. The reaction mixture was cooled down to $-78{ }^{\circ} \mathrm{C}$ and a cold solution of $m$-CPBA ( $50 \mathrm{mg}, 220 \mu \mathrm{mol}, 2$ equiv) in $1 \mathrm{~mL}$ of dry DCM dropwise at $-78^{\circ} \mathrm{C}$. After $1 \mathrm{~h}$ at -78 ${ }^{\circ} \mathrm{C}$, the reaction mixture was quenched with a saturated solution of $\mathrm{Na}_{2} \mathrm{~S}_{2} \mathrm{O}_{3}(5 \mathrm{~mL})$ and then allowed to warmed up to rt. The organic layer was washed with cold $\mathrm{NaHCO}_{3}(5 \mathrm{~mL})$, cold water $(5 \mathrm{~mL})$ and cold brine $(5 \mathrm{~mL})$, dried over $\mathrm{Na}_{2} \mathrm{SO}_{4}$, filtered and transferred into a flame-dry sealed tube with a catalytic amount of BHT ( 0.1 equiv). The resulting solution was heated at $100{ }^{\circ} \mathrm{C}$ overnight before being cooled down to rt and concentrated in vacuo. The crude product was purified via silica gel chromatography ( $\mathrm{PE} / \mathrm{Et}_{2} \mathrm{O} 20: 1$ to $9: 1$ ) to yield $17.7 \mathrm{mg}$ (38\% yield) of endo-tetracyclic product 24 and $12.5 \mathrm{mg}$ ( $27 \%$ yield) of exo-tetracyclic product $\mathbf{2 5}$, both as yellowish resins.

Data for 24:

$[\alpha]_{D}^{25}=+93\left(\mathrm{c} 0.2, \mathrm{CHCl}_{3}\right)$.

${ }^{1}$ H NMR (400 MHz, $\left.\mathrm{CDCl}_{3}\right) \delta$ ppm: 7.50-7.45 (m, 3H), 7.40-7.34 (m, 2H), 5.78 (brs, $\left.1 \mathrm{H}\right), 4.33-4.30$ (m, $1 \mathrm{H}), 3.56-3.48(\mathrm{~m}, 1 \mathrm{H}), 3.35$ (brs, $1 \mathrm{H}), 2.75(\mathrm{dd}, J=6.3,1.8 \mathrm{~Hz}, 1 \mathrm{H}), 2.69$ (dd, $J=16.5,5.5 \mathrm{~Hz}, 1 \mathrm{H}), 2.56-$ $2.44(\mathrm{~m}, 1 \mathrm{H}), 2.30(\mathrm{dd}, J=16.6,11.3 \mathrm{~Hz}), 2.20-2.09(\mathrm{~m}, 2 \mathrm{H}), 1.85-1.79(\mathrm{~m}, 1 \mathrm{H}), 1.79(\mathrm{~s}, 3 \mathrm{H}), 1.72-1.67$ $(\mathrm{m}, 2 \mathrm{H}), 1.65(\mathrm{~s}, 3 \mathrm{H}), 1.64-1.56(\mathrm{~m}, 3 \mathrm{H}), 1.54-1.47(\mathrm{~m}, 3 \mathrm{H}), 1.31(\mathrm{~d}, J=7.3 \mathrm{~Hz}, 3 \mathrm{H}), 0.99(\mathrm{~d}, J=4.5 \mathrm{~Hz}$, $3 \mathrm{H}), 0.98(\mathrm{~d}, J=4.5 \mathrm{~Hz}, 3 \mathrm{H})$.

${ }^{13} \mathrm{C}$ NMR $\left(100 \mathrm{MHz}, \mathrm{CDCl}_{3}\right) \delta$ ppm: 208.4, 171.7, 170.5, 137.1, 135.1, 134.8, 133.4, 132.1, 129.8, 128.7, 128.0, 69.6, 54.4, 47.7, 46.0, 44.7, 43.2, 37.3, 35.0, 34.5, 33.7, 27.3, 24.7, 24.4, 24.2, 24.1, 21.9, 19.4, 13.4 .

IR (ATR) v cm${ }^{-1}:$ 2959, 2928, 2868, 2857, 1736, 1701, 1672, 1653, 1448, 1287, 1221, 1207, 1161, 696, 660.

HRMS (ESI+) $\mathrm{m} / \mathrm{z}$ : calculated for $\mathrm{C}_{31} \mathrm{H}_{40} \mathrm{NO}_{3}{ }^{+}\left[\mathrm{MH}^{+}\right]:$: 474.3003; found: 474.3022 .

Data for 25: 
$[\alpha]_{D}^{25}=+234\left(\mathrm{c} 0.2, \mathrm{CHCl}_{3}\right)$.

${ }^{1} \mathrm{H}$ NMR $\left(400 \mathrm{MHz}, \mathrm{CDCl}_{3}\right) \delta$ ppm: 7.77-7.73 (m, 2H), 7.52-7.48 (m, $\left.1 \mathrm{H}\right)$, 7.41-7.37 $(\mathrm{m}, 2 \mathrm{H})$, 5.48-5.45 $(\mathrm{m}, 1 \mathrm{H}), 4.24-4.20(\mathrm{~m}, 1 \mathrm{H}), 3.51-3.47(\mathrm{~m}, 1 \mathrm{H}), 2.92-2.84(\mathrm{~m}, 1 \mathrm{H}), 2.69(\mathrm{t}, J=13.2 \mathrm{~Hz}, 1 \mathrm{H}), 2.32(\mathrm{~d}, J=$ 4.3 Hz, $1 \mathrm{H}), 2.31-2.24(\mathrm{~m}, 2 \mathrm{H}), 2.17-2.09(\mathrm{~m}, 1 \mathrm{H}), 2.00-1.84(\mathrm{~m}, 2 \mathrm{H}), 1.80(\mathrm{~s}, 3 \mathrm{H}), 1.78-1.71(\mathrm{~m}, 1 \mathrm{H})$, 1.71-1.66 (m, 2H), $1.63(\mathrm{~d}, J=1.6 \mathrm{~Hz}, 3 \mathrm{H}), 1.62-1.54(\mathrm{~m}, 1 \mathrm{H}), 1.60-1.46(\mathrm{~m}, 1 \mathrm{H}), 1.36-1.28(\mathrm{~m}, 1 \mathrm{H}), 1.20-$ $1.16(\mathrm{~m}, 1 \mathrm{H}), 1.13(\mathrm{~d}, J=7.4 \mathrm{~Hz}, 3 \mathrm{H}), 0.97(\mathrm{~d}, J=6.4 \mathrm{~Hz}, 3 \mathrm{H}), 0.92(\mathrm{~d}, J=6.4 \mathrm{~Hz}, 3 \mathrm{H})$.

${ }^{13} \mathrm{C}$ NMR $\left(100 \mathrm{MHz}, \mathrm{CDCl}_{3}\right) \delta$ ppm: 211.6, 173.4, 171.7, 137.1, 136.6, 134.3, 135.9, 132.8, 129.8, 128.0, 125.3, 60.4, 57.9, 49.5, 45.2, 42.6, 39.4, 38.9, 36.1, 33.1, 31.7, 26.9, 25.1, 24.8, 23.5, 23.0, 21.6, 21.3, 18.5 .

IR ( $\mathrm{KBr}$ pellets) $\vee \mathrm{cm}^{-1}:$ 2957, 2922, 2851, 1740, 1736, 1701, 1684, 1653, 1448, 1283, 1244, 1201, 1163, 721, 694, 660;

HRMS (ESI+) $\mathrm{m} / \mathrm{z}$ : calculated for $\mathrm{C}_{31} \mathrm{H}_{40} \mathrm{NO}_{3}{ }^{+}\left[\mathrm{MH}^{+}\right]:$: 474.3003; found: 474.3005 .

(3S,3aR,4S,6aS,8aS,13bR)-4-Isobutyl-2,3,13-trimethyl-4,5,8,8a,9,10,11,12-octahydro-3Hcyclohepta[3,4]benzo[1,2-d]isoindole-6,7(3aH,13bH)-dione (5)

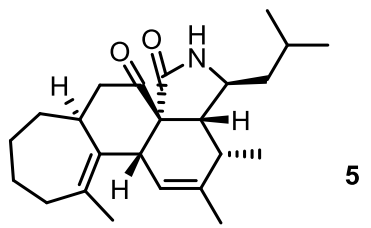

To a stirred solution of tetracyclic cycloadduct 24 (17.7 mg, $37 \mu \mathrm{mol}, 1$ equiv) in $0.8 \mathrm{~mL}$ of MeOH was added a $14.8 \mathrm{M}$ solution of $\mathrm{NaOH}$ in water $\left(10 \mu \mathrm{L}, 150 \mu \mathrm{mol}, 4\right.$ equiv) at $0{ }^{\circ} \mathrm{C}$. The reaction mixture was allowed to warm up to rt over $2 \mathrm{~h}$. The reaction mixture was quenched with a water $(0.5 \mathrm{~mL})$. The aqueous layer was extracted with $\mathrm{Et}_{2} \mathrm{O}(3 \times 0.5 \mathrm{~mL})$. The organic layers were combined, washed with brine, dried over $\mathrm{Na}_{2} \mathrm{SO}_{4}$, filtered and concentrated in vacuo. The crude product was purified via silica gel chromatography ( $\left.\mathrm{DCM} / \mathrm{Et}_{2} \mathrm{O} 95: 5\right)$ to yield $11.8 \mathrm{mg}$ (87\% yield) of deprotected cycloadduct 5 as a colorless resin.

${ }^{1} \mathrm{H}$ NMR $\left(400 \mathrm{MHz}, \mathrm{CDCl}_{3}\right) \delta$ ppm: $5.94(\mathrm{~s}, 1 \mathrm{H}), 5.58(\mathrm{~s}, 1 \mathrm{H}), 3.64-3.53(\mathrm{~m}, 1 \mathrm{H}), 3.28(\mathrm{~s}, \mathrm{brs}), 3.16$ (td, $J=$ 10.1, 3.5 Hz, $1 \mathrm{H}$ ), 2.69 (dd, $J=5.3,3.5 \mathrm{~Hz}, 1 \mathrm{H}), 2.56(\mathrm{dd}, J=17.3,5.5 \mathrm{~Hz}, 1 \mathrm{H}), 2.42-2.28(\mathrm{~m}, 2 \mathrm{H}), 2.28$ (dd, $1 \mathrm{H}, J=17.3,12.2 \mathrm{~Hz}, 1 \mathrm{H}), 2.19-2.08(\mathrm{~m}, 1 \mathrm{H}), 1.88-1.79(\mathrm{~m}, 1 \mathrm{H}), 1.76(\mathrm{~s}, 3 \mathrm{H}), 1.73(\mathrm{~s}, 3 \mathrm{H}), 1.71-1.41$ $(\mathrm{m}, 6 \mathrm{H}), 1.36-1.28(\mathrm{~m}, 1 \mathrm{H}), 1.17(\mathrm{~d}, J=7.2 \mathrm{~Hz}, 3 \mathrm{H}), 0.97(\mathrm{~d}, J=6.8 \mathrm{~Hz}, 3 \mathrm{H}), 0.92(\mathrm{~d}, J=6.8 \mathrm{~Hz}, 3 \mathrm{H})$.

${ }^{13} \mathrm{C}$ NMR $\left(100 \mathrm{MHz}, \mathrm{CDCl}_{3}\right) \delta \mathrm{ppm:} 210.5,173.3,136.9,134.9,133.6,128.5,65.8,51.2,51.1,47.8,47.2$, 41.6, 36.6, 35.7, 34.7, 33.5, 28.2, 24.2, 23.7, 21.5, 20.2, 13.6.

IR (ATR) v cm ${ }^{-1}:$ 2957, 2922, 2851, 1688, 1653, 1554, 1273, 1264, 745, 696, 664.

HRMS (ESI+) m/z: calculated for $\mathrm{C}_{24} \mathrm{H}_{36} \mathrm{NO}_{2}{ }^{+}\left[\mathrm{MH}^{+}\right]: 370.2741$; found: 370.2730 .

(3S,3aR,4S,6aS,8aS,13R,13aR,13bR)-5-Benzoyl-13,13a-epoxy-4-isobutyl-2,3,13-trimethyl4,5,8,8a,9,10,11,12-octahydro-3H-cyclohepta[3,4]benzo[1,2-d]isoindole-6,7(3aH,13bH)-dione (26) and 
(3S,3aR,4S,6aS,8aS,13S,13aS,13bR)-5-Benzoyl-13,13a-epoxy-4-isobutyl-2,3,13-trimethyl4,5,8,8a,9,10,11,12-octahydro-3H-cyclohepta[3,4]benzo[1,2-d]isoindole-6,7(3aH,13bH)-dione (27)

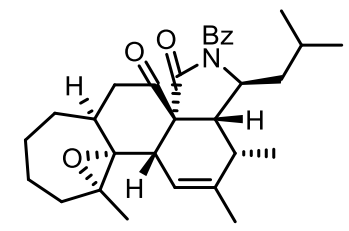

26
Major stereoisomer

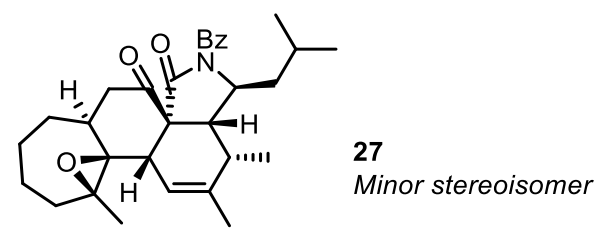

To a stirred solution of tetracyclic product 24 ( $6 \mathrm{mg}, 12.7 \mu \mathrm{mol}, 1$ equiv) and $\mathrm{NaHCO}_{3}(1 \mathrm{mg}, 12 \mu \mathrm{mol}$, 1 equiv) in $0.3 \mathrm{~mL}$ of DCM was added a solution of $\mathrm{m}$-CPBA ( $2.8 \mathrm{mg}, 12 \mu \mathrm{mol}, 1$ equiv) in $0.1 \mathrm{~mL}$ of $\mathrm{DCM}$. The reaction mixture was allowed to warm up to $-10{ }^{\circ} \mathrm{C}$ during $2 \mathrm{~h}$ before being quenched with a saturated solution of $\mathrm{Na}_{2} \mathrm{~S}_{2} \mathrm{O}_{3}(1 \mathrm{~mL})$. The reaction was allowed to warm up to $\mathrm{rt}$ and the organic layer was washed with a saturated solution of $\mathrm{NaHCO}_{3}(1 \mathrm{~mL})$, brine $(1 \mathrm{~mL})$, dried with $\mathrm{Na}_{2} \mathrm{SO}_{4}$, filtered and con- centrated in vacuo. The crude product was purified via silica gel chromatography (PE/EA 9:1) to yield $3 \mathrm{mg}$ of 26 (48\%) and $1.4 \mathrm{mg}$ of $\mathbf{2 7}(22 \%)$, as colorless resins.

Data for 26 (major isomer):

$[\alpha]_{D}^{25}=+3.2\left(\mathrm{c} 0.53, \mathrm{CHCl}_{3}\right)$.

7.63-7.58 (m, 2H), 7.09-7.02 (m, 3H), 5.84-5.89 (m, 1H), 4.55-4.50 (m, 1H), 3.96 (dd, J = 12.1, $6.8 \mathrm{~Hz}$, $1 \mathrm{H}), 3.14(\mathrm{~d}, J=6.6 \mathrm{~Hz}, 1 \mathrm{H}), 2.81-2.76(\mathrm{~m}, 1 \mathrm{H}), 2.31-2.23\left(\mathrm{~m}, 1 \mathrm{H}, \mathrm{H}_{16}\right), 2.03(\mathrm{t}, J=7.2 \mathrm{~Hz} 1 \mathrm{H}), 1.96(\mathrm{dd}, J$ $=12.1,2.4 \mathrm{~Hz}, 1 \mathrm{H}), 1.81-1.59(\mathrm{~m}, 4 \mathrm{H}), 1.58-1.55(\mathrm{~m}, 3 \mathrm{H}), 1.54-1.25(\mathrm{~m}, 8 \mathrm{H}), 1.14(\mathrm{~d}, \mathrm{~J}=0.8 \mathrm{~Hz}, 3 \mathrm{H}), 1.04$ $(\mathrm{d}, J=6.4 \mathrm{~Hz}, 9 \mathrm{H})$.

${ }^{13} \mathrm{C}$ NMR (100 MHz, C $\left.6 \mathrm{D}_{6}\right) \delta$ ppm: 204.1, 171.4, 170.0, 137.5, 135.5, 131.9, 129.1, 128.4, 124.9, 71.4, 64.0, 61.2, 54.7, 45.8, 45.0, 44.2, 43.6, 41.4, 38.4, 34.3, 34.0, 29.1, 25.1, 24.1, 23.9, 21.9, 19.4, 18.0, 13.4 .

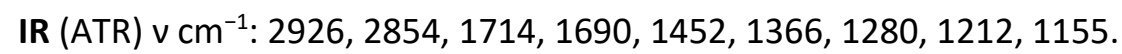

HRMS (ESI+) $\mathrm{m} / \mathrm{z}$ : calculated for $\mathrm{C}_{31} \mathrm{H}_{40} \mathrm{NO}_{3}{ }^{+}\left[\mathrm{MH}^{+}\right]: 490.2952$ found: 490.2953.

Data for 27 (minor isomer):

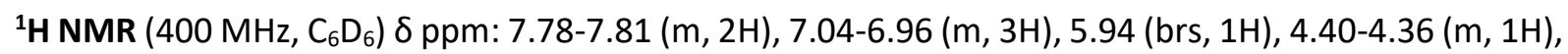
3.15-3.11 (m, $1 \mathrm{H}), 2.79(\mathrm{t}, J=4.9 \mathrm{~Hz}, 1 \mathrm{H}), 2.52-2.44(\mathrm{~m}, 2 \mathrm{H}), 2.36-2.30(\mathrm{~m}, 1 \mathrm{H}), 1.85-1.71(\mathrm{~m}, 4 \mathrm{H}), 1.68-$ $1.51(\mathrm{~m}, 5 \mathrm{H}), 1.57$ (brs, 3H), 1.51-1.47 (m, 2H), 1.27-1.21 (m, $1 \mathrm{H}), 1.24(\mathrm{~s}, 3 \mathrm{H}), 1.00(\mathrm{~d}, J=5.9 \mathrm{~Hz}, 3 \mathrm{H})$, $0.93(d, J=7.4 \mathrm{~Hz}, 3 \mathrm{H}), 0.89(\mathrm{~d}, \mathrm{~J}=5.9 \mathrm{~Hz}, 3 \mathrm{H})$.

${ }^{13} \mathrm{C}$ NMR (100 MHz, $\left.\mathrm{C}_{6} \mathrm{D}_{6}\right) \delta \mathrm{ppm}: 208.4,172.4,170.8,138.0,135.1,132.6,130.0,128.3,122.9,66.1$, 66.0, 65.2, 56.0, 48.5, 47.5, 46.6, 42.2, 35.8, 35.1, 34.8, 29.7, 29.6, 25.0, 24.0, 23.6, 22.7, 21.3, 20.5, 14.7.

HRMS (ESI+) m/z: calculated for $\mathrm{C}_{31} \mathrm{H}_{40} \mathrm{NO}_{3}{ }^{+}\left[\mathrm{MH}^{+}\right]$: 490.2952 found: 490.2953.

\section{X-Ray crystallography of epoxide 26}

Suitable single crystals were selected and mounted on a Bruker APEX-II CCD diffractometer. The crystals were kept at $150 \mathrm{~K}$ during data collection. 
Using Olex $2{ }^{7}$ the structure was solved with the SHELXT ${ }^{8}$ structure solution program using Intrinsic Phasing and refined with the SHELXL ${ }^{9}$ refinement package using Least Squares minimization. The PLATON software ${ }^{10}$ was used to treat cases of solvent accessible voids. Pictures of the compound structure were obtained using the MERCURY software. During the refinement steps, all atoms, except hydrogen atoms, were refined anisotropically. The positions of the hydrogen atoms were determined geometrically. The crystal structure of $\mathbf{2 6}$ was deposited on the Cambridge Crystallographic Data Centre under CCDC deposition number 2086360.

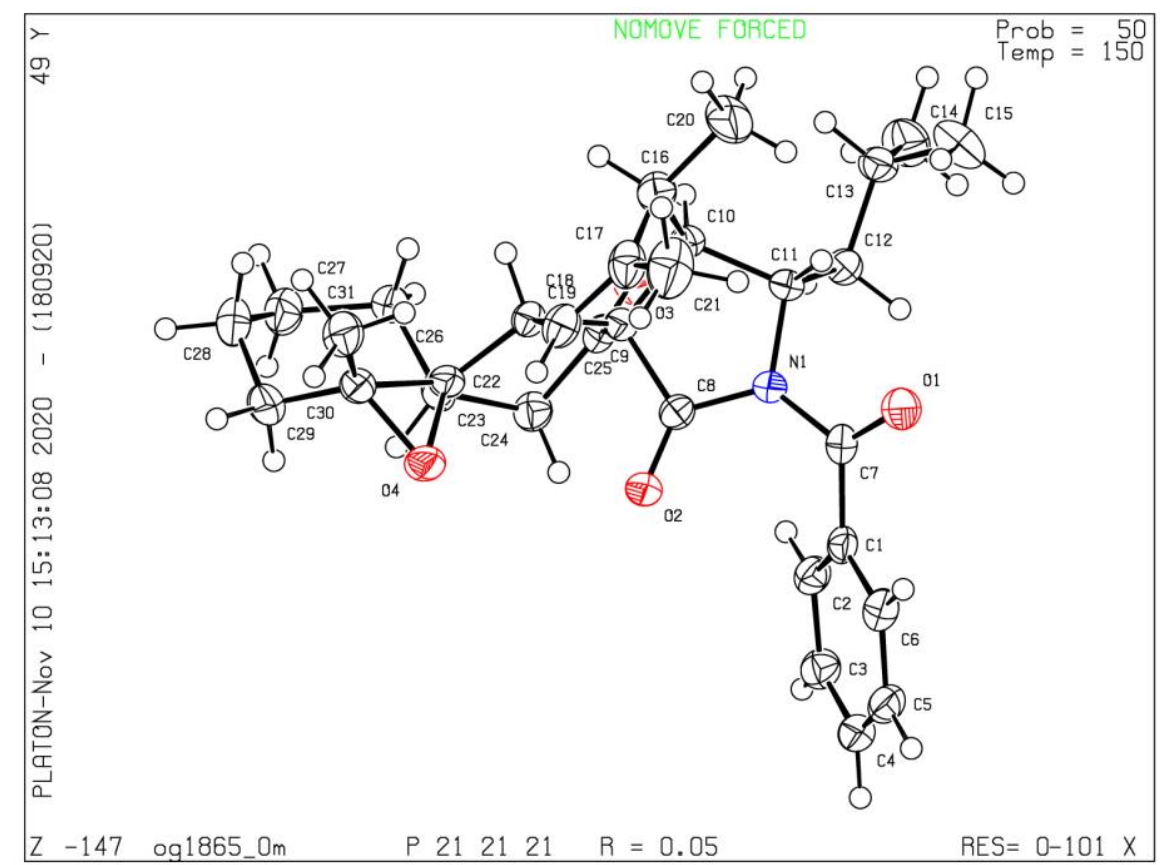

Figure S1. Epsilon plot of compound 26, atom numbering.

Table 1. Crystal data and structure refinement for 26.

Identification code

og1865_0m

Empirical formula

$\mathrm{C}_{31} \mathrm{H}_{39} \mathrm{NO}_{4}$

Formula weight

489.63

Temperature/K

150.01

Crystal system

orthorhombic

Space group

$\mathrm{P} 2{ }_{1} 2_{1} 2_{1}$

$a / \AA ̊$

8.8890(19)

b/Å

$10.515(2)$

\footnotetext{
${ }^{7}$ Dolomanov, O.V.; Bourhis, L.J; Gildea, R.J.; Howard, J.A.K.; Puschmann, H. J. Appl. Cryst. 2009, 42, 339.

${ }^{8}$ Sheldrick, G.M. Acta Cryst. 2015, A71, 3 .

${ }^{9}$ Sheldrick, G.M. Acta Cryst. 2015, C71, 3 .

${ }^{10}$ Spek, A. J. App. Crystallogr. 2003, 36, 7.
} 
$c / \AA$

$\alpha /^{\circ}$

$\beta /{ }^{\circ}$

$\gamma /{ }^{\circ}$

Volume/ $/ \AA^{3}$

Z

$\rho_{\text {calc }} \mathrm{g} / \mathrm{cm}^{3}$

$\mu / \mathrm{mm}^{-1}$

$F(000)$

Crystal size $/ \mathrm{mm}^{3}$

Radiation

$2 \Theta$ range for data collection/ ${ }^{\circ}$

Index ranges

Reflections collected

Independent reflections

Data/restraints/parameters

Goodness-of-fit on $\mathrm{F}^{2}$

Final $R$ indexes $[\mid>=2 \sigma(I)]$

Final $\mathrm{R}$ indexes [all data]

Largest diff. peak/hole / e $\AA^{-3}$

Flack parameter
$28.332(6)$

90

90

90

2648.1(10)

4

1.228

0.080

1056.0

$0.18 \times 0.1 \times 0.02$

$\operatorname{MoK} \alpha(\lambda=0.71073)$

4.802 to 52.04

$-10 \leq h \leq 10,-11 \leq k \leq 12,-34 \leq \mathrm{I} \leq 33$

27793

$5191\left[R_{\text {int }}=0.0989, R_{\text {sigma }}=0.0732\right]$

$5191 / 0 / 331$

1.046

$\mathrm{R}_{1}=0.0492, \mathrm{wR}_{2}=0.1110$

$\mathrm{R}_{1}=0.0765, \mathrm{wR} \mathrm{R}_{2}=0.1253$

$0.22 /-0.29$

$-0.4(17)$

Table 2. Fractional Atomic Coordinates $\left(\times 10^{4}\right)$ and Equivalent Isotropic Displacement Parameters $\left(\AA^{2} \times 10^{3}\right)$ for 26 . $U_{\text {eq }}$ is defined as $1 / 3$ of of the trace of the orthogonalised $U_{\text {IJ }}$ tensor.

\begin{tabular}{lllll} 
Atom & $\boldsymbol{x}$ & $\boldsymbol{y}$ & $\boldsymbol{z}$ & $\mathrm{U}(\mathrm{eq})$ \\
\hline C1 & $1234(4)$ & $7428(3)$ & $5873.6(11)$ & $26.5(7)$ \\
C2 & $272(4)$ & $6471(3)$ & $5725.8(12)$ & $30.3(8)$
\end{tabular}




\begin{tabular}{|c|c|c|c|c|}
\hline C3 & $-633(4)$ & $6643(3)$ & $5334.1(12)$ & $35.1(8)$ \\
\hline C4 & $-543(4)$ & 7765(3) & $5078.9(13)$ & 36.0(9) \\
\hline C5 & $407(4)$ & $8726(3)$ & $5220.4(13)$ & $35.3(8)$ \\
\hline C6 & $1283(4)$ & $8568(3)$ & $5620.9(12)$ & $31.9(8)$ \\
\hline C7 & $2134(4)$ & 7320(3) & 6312.1(12) & 27.3(7) \\
\hline C8 & $3216(4)$ & $5197(3)$ & 6144.7(11) & $25.5(7)$ \\
\hline C9 & $3860(4)$ & $4108(3)$ & 6442.7(11) & $25.0(7)$ \\
\hline C10 & 4029(4) & 4689(3) & 6941.2(11) & $26.8(7)$ \\
\hline C11 & 2941(4) & $5832(3)$ & 6949.4(11) & 26.0(7) \\
\hline C12 & $1456(4)$ & $5559(3)$ & 7204.8(11) & $29.7(8)$ \\
\hline C13 & $1619(4)$ & $5467(3)$ & 7745.0(11) & $32.6(8)$ \\
\hline C14 & 285(5) & 4789(4) & 7962.4(14) & 47.1(10) \\
\hline C15 & $1807(5)$ & 6768(4) & 7965.0(14) & $50.1(11)$ \\
\hline C16 & $5702(4)$ & $5040(4)$ & $7044.5(12)$ & 39.9(9) \\
\hline C17 & $6422(4)$ & $5615(3)$ & 6612.7(13) & $38.3(9)$ \\
\hline C18 & $6308(4)$ & 4924(3) & 6215.7(13) & $33.7(8)$ \\
\hline C19 & $5427(4)$ & $3703(3)$ & $6238.9(12)$ & 27.1(7) \\
\hline $\mathrm{C} 20$ & $5871(5)$ & $5794(5)$ & $7505.9(14)$ & $60.2(13)$ \\
\hline C21 & 7178(5) & 6891(4) & 6627.6(18) & $57.3(12)$ \\
\hline C22 & $5406(4)$ & $2824(3)$ & $5805.8(11)$ & 27.1(7) \\
\hline C23 & 4140(4) & 1849(3) & $5804.9(12)$ & 29.5(8) \\
\hline C24 & $2602(4)$ & 2411(3) & $5942.4(12)$ & $31.5(8)$ \\
\hline C25 & $2732(4)$ & 3010(3) & $6420.3(12)$ & $28.5(8)$ \\
\hline C26 & $4564(4)$ & $728(3)$ & $6128.9(12)$ & $31.6(8)$ \\
\hline C27 & $5427(5)$ & $-322(3)$ & $5875.3(14)$ & 41.7(9) \\
\hline C28 & $6943(5)$ & $43(3)$ & $5668.3(14)$ & $45.2(10)$ \\
\hline C29 & $6917(4)$ & 1162(3) & $5324.7(13)$ & 39.3(9) \\
\hline
\end{tabular}




$\begin{array}{lllll}\text { C30 } & 6825(4) & 2442(3) & 5569.0(11) & 32.3(8) \\ \text { C31 } & 8350(4) & 2929(4) & 5717.1(14) & 39.7(9) \\ \mathrm{N} 1 & 2640(3) & 6098(2) & 6445.6(9) & 25.5(6) \\ 01 & 2399(3) & 8217(2) & 6566.3(9) & 38.4(6) \\ 02 & 3233(3) & 5244(2) & 5716.7(8) & 31.1(5) \\ 03 & 2649(2) & 6763.6(9) & 41.2(6) \\ 04 & 2026(3) & 3374(2) & 5355.1(8) & 35.9(6)\end{array}$

Table 3. Anisotropic Displacement Parameters $\left(\AA^{2} \times 10^{3}\right)$ for 26. The Anisotropic displacement factor exponent takes the form: $-2 \pi^{2}\left[h^{2} a * 2 U_{11}+2 h k a * b * U_{12}+\ldots\right]$.

\begin{tabular}{|c|c|c|c|c|c|c|}
\hline Atom & $\mathbf{U}_{11}$ & $U_{22}$ & $U_{33}$ & $U_{23}$ & $\mathrm{U}_{13}$ & $U_{12}$ \\
\hline $\mathrm{C} 1$ & $24.0(16)$ & $27.0(17)$ & $28.6(18)$ & $-0.7(15)$ & 4.3(14) & $6.2(14)$ \\
\hline C2 & $31.2(19)$ & $26.6(17)$ & $33.2(19)$ & $3.4(15)$ & $1.4(16)$ & $2.9(15)$ \\
\hline C3 & $30.9(19)$ & $37(2)$ & $37(2)$ & $-2.5(16)$ & $-3.8(17)$ & $2.4(16)$ \\
\hline $\mathrm{C} 4$ & $36(2)$ & $42(2)$ & $30(2)$ & $0.4(16)$ & $-0.2(17)$ & 11.7(18) \\
\hline C5 & $37.1(19)$ & $36(2)$ & $32.9(19)$ & $8.6(16)$ & $5.3(17)$ & 11.6(17) \\
\hline C6 & $30.0(19)$ & $27.5(18)$ & $38(2)$ & $3.4(16)$ & $6.2(17)$ & $2.7(15)$ \\
\hline $\mathrm{C7}$ & $26.4(17)$ & $23.4(17)$ & $31.9(18)$ & $-2.0(15)$ & 4.6(15) & $2.2(14)$ \\
\hline C8 & $22.3(16)$ & $26.3(16)$ & $27.9(18)$ & $1.2(14)$ & $-1.1(15)$ & $-0.1(14)$ \\
\hline C9 & $24.9(17)$ & $27.8(16)$ & $22.5(16)$ & $0.2(14)$ & $0.2(14)$ & $2.8(13)$ \\
\hline C10 & $25.1(17)$ & $31.8(17)$ & $23.6(17)$ & $0.3(15)$ & $-1.8(14)$ & $3.0(14)$ \\
\hline C11 & $27.7(18)$ & $29.8(17)$ & $20.4(16)$ & $-0.8(14)$ & $-2.3(14)$ & $2.3(14)$ \\
\hline $\mathrm{C} 12$ & $24.8(17)$ & $35.4(18)$ & $28.8(18)$ & $-1.9(15)$ & $-1.8(15)$ & $3.5(15)$ \\
\hline $\mathrm{C} 13$ & $30.3(18)$ & $43(2)$ & $24.5(17)$ & $1.4(16)$ & $0.7(16)$ & $3.7(17)$ \\
\hline C14 & $43(2)$ & $57(3)$ & $41(2)$ & 4.0(19) & $12.0(19)$ & $-2(2)$ \\
\hline C15 & $56(3)$ & $61(3)$ & $33(2)$ & $-10.7(19)$ & $7(2)$ & $-13(2)$ \\
\hline C16 & $32(2)$ & $48(2)$ & $40(2)$ & $-13.1(18)$ & $-12.0(18)$ & $9.4(18)$ \\
\hline
\end{tabular}


Table 3. Anisotropic Displacement Parameters $\left(\AA^{2} \times 10^{3}\right)$ for 26. The Anisotropic displacement factor exponent takes the form: $-2 \pi^{2}\left[h^{2} a * 2 U_{11}+2 h k a * b * U_{12}+\ldots\right]$.

\begin{tabular}{|c|c|c|c|c|c|c|}
\hline Atom & $\mathrm{U}_{11}$ & $U_{22}$ & $U_{33}$ & $U_{23}$ & $U_{13}$ & $U_{12}$ \\
\hline C17 & $27(2)$ & $38(2)$ & $50(2)$ & $-8.7(18)$ & $-0.2(17)$ & $3.0(16)$ \\
\hline $\mathrm{C} 18$ & $21.9(17)$ & $35.7(19)$ & $44(2)$ & $0.2(17)$ & $0.8(17)$ & $2.1(15)$ \\
\hline C19 & $22.5(16)$ & $31.1(18)$ & $27.8(18)$ & $0.2(14)$ & $0.1(15)$ & $4.4(14)$ \\
\hline $\mathrm{C} 20$ & $43(2)$ & $89(3)$ & $48(3)$ & $-32(2)$ & $-21(2)$ & $15(2)$ \\
\hline $\mathrm{C} 21$ & $43(3)$ & $45(2)$ & $84(3)$ & $-17(2)$ & $-7(2)$ & $-2(2)$ \\
\hline $\mathrm{C} 22$ & $28.7(17)$ & $30.8(17)$ & $21.8(17)$ & $4.6(13)$ & 2.1(15) & $3.9(15)$ \\
\hline $\mathrm{C} 23$ & $29.8(18)$ & $31.1(18)$ & 27.7(19) & $-6.6(15)$ & $1.0(16)$ & $1.8(15)$ \\
\hline $\mathrm{C} 24$ & $25.5(17)$ & $33.8(19)$ & $35(2)$ & $-2.6(16)$ & $-2.9(16)$ & $-0.3(15)$ \\
\hline $\mathrm{C} 25$ & $24.5(17)$ & $27.0(17)$ & $34(2)$ & $1.3(15)$ & $0.9(16)$ & $6.0(14)$ \\
\hline C26 & $33.3(19)$ & $31.1(18)$ & $30.3(18)$ & $-0.5(15)$ & $-1.2(16)$ & $1.4(15)$ \\
\hline $\mathrm{C} 27$ & $47(2)$ & $31.5(19)$ & $46(2)$ & $-2.7(17)$ & $2(2)$ & $6.2(19)$ \\
\hline $\mathrm{C} 28$ & $49(2)$ & $40(2)$ & $47(2)$ & $-5.4(19)$ & $6(2)$ & $16.2(19)$ \\
\hline $\mathrm{C} 29$ & $38(2)$ & $50(2)$ & $30.2(19)$ & $-3.6(17)$ & $6.0(18)$ & $9.7(18)$ \\
\hline C30 & $33.0(19)$ & $40(2)$ & 23.9(17) & $4.5(16)$ & $5.5(16)$ & $8.8(16)$ \\
\hline C31 & 29.1(19) & $49(2)$ & $41(2)$ & $2.5(18)$ & $9.0(18)$ & 4.9(17) \\
\hline N1 & $28.4(15)$ & $26.8(14)$ & $21.4(14)$ & $-0.6(11)$ & $-2.6(12)$ & $3.3(12)$ \\
\hline 01 & $49.7(16)$ & $28.7(13)$ & $36.7(14)$ & $-4.2(11)$ & $-4.5(12)$ & $3.6(12)$ \\
\hline $\mathrm{O} 2$ & $35.6(13)$ & $35.3(13)$ & $22.5(12)$ & $2.6(10)$ & $1.2(11)$ & $7.2(11)$ \\
\hline $\mathrm{O} 3$ & $43.7(15)$ & $40.4(14)$ & $39.5(15)$ & $1.1(12)$ & $16.7(13)$ & $-4.3(13)$ \\
\hline $\mathrm{O} 4$ & $36.7(14)$ & $45.2(14)$ & $25.7(12)$ & $10.4(11)$ & $6.0(11)$ & $8.6(12)$ \\
\hline
\end{tabular}

Table 4. Bond Lengths for 26.

Atom Atom Length/Å Atom Atom Length/Å

$\begin{array}{llllll}C 1 & \text { C2 } & 1.386(5) & \text { C13 } & \text { C15 } & 1.512(5)\end{array}$ 
Table 4. Bond Lengths for 26.

Atom Atom Length/Å Atom Atom Length/Å

\begin{tabular}{|c|c|c|c|c|c|}
\hline C1 & C6 & $1.396(5)$ & C16 & C17 & $1.507(5)$ \\
\hline C1 & C7 & $1.482(5)$ & C16 & C20 & $1.536(5)$ \\
\hline $\mathrm{C} 2$ & C3 & $1.383(5)$ & C17 & C18 & $1.343(5)$ \\
\hline C3 & C4 & $1.386(5)$ & C17 & C21 & $1.502(5)$ \\
\hline C4 & C5 & $1.376(5)$ & C18 & C19 & $1.505(5)$ \\
\hline C5 & C6 & $1.386(5)$ & C19 & $\mathrm{C} 22$ & $1.536(5)$ \\
\hline C7 & N1 & $1.413(4)$ & C22 & $\mathrm{C} 23$ & $1.522(5)$ \\
\hline C7 & 01 & $1.210(4)$ & $\mathrm{C} 22$ & C30 & $1.483(5)$ \\
\hline C8 & C9 & $1.534(4)$ & $\mathrm{C} 22$ & $\mathrm{O} 4$ & $1.448(4)$ \\
\hline C8 & N1 & $1.373(4)$ & $\mathrm{C} 23$ & C24 & $1.539(5)$ \\
\hline C8 & $\mathrm{O} 2$ & $1.214(4)$ & $\mathrm{C} 23$ & C26 & $1.541(5)$ \\
\hline C9 & C10 & $1.546(4)$ & C24 & $\mathrm{C} 25$ & $1.498(5)$ \\
\hline C9 & C19 & $1.567(4)$ & $\mathrm{C} 25$ & $\mathrm{O} 3$ & $1.218(4)$ \\
\hline C9 & $\mathrm{C} 25$ & $1.530(4)$ & $\mathrm{C} 26$ & $\mathrm{C} 27$ & $1.524(5)$ \\
\hline C10 & C11 & $1.543(4)$ & $\mathrm{C} 27$ & $\mathrm{C} 28$ & $1.520(6)$ \\
\hline C10 & C16 & $1.560(5)$ & C28 & C29 & $1.527(5)$ \\
\hline C11 & $\mathrm{C} 12$ & $1.532(5)$ & C29 & C30 & $1.516(5)$ \\
\hline C11 & N1 & $1.479(4)$ & C30 & C31 & $1.508(5)$ \\
\hline C12 & C13 & $1.540(5)$ & C30 & $\mathrm{O} 4$ & $1.461(4)$ \\
\hline C13 & C14 & $1.514(5)$ & & & \\
\hline
\end{tabular}

Table 5. Bond Angles for 26.

\begin{tabular}{lllllllll}
\multicolumn{2}{c}{ Atom Atom Atom Angle/ } & \multicolumn{4}{c}{ Atom Atom Atom Angle/ } \\
\hline C2 & C1 & C6 & $119.2(3)$ & C18 & C17 & C21 & $122.8(4)$ \\
C2 & C1 & C7 & $122.0(3)$ & C21 & C17 & C16 & $121.7(3)$
\end{tabular}


Table 5. Bond Angles for 26.

\begin{tabular}{|c|c|c|c|c|c|c|c|}
\hline \multicolumn{4}{|c|}{ Atom Atom Atom Angle/ ${ }^{\circ}$} & \multicolumn{4}{|c|}{ Atom Atom Atom Angle/ ${ }^{\circ}$} \\
\hline C6 & $\mathrm{C} 1$ & $\mathrm{C7}$ & $118.6(3)$ & C17 & C18 & C19 & $117.7(3)$ \\
\hline C3 & $\mathrm{C} 2$ & $\mathrm{C} 1$ & & & & C9 & \\
\hline $\mathrm{C} 2$ & C3 & C4 & & & & & $119.0(3)$ \\
\hline C5 & C4 & C3 & & & & C9 & \\
\hline $\mathrm{C} 4$ & C5 & C6 & & $\mathrm{C} 23$ & $\mathrm{C} 22$ & C19 & $114.6(3)$ \\
\hline C5 & C6 & $\mathrm{C} 1$ & & C30 & $\mathrm{C} 22$ & C19 & $120.9(3)$ \\
\hline N1 & $\mathrm{C7}$ & $\mathrm{C} 1$ & & C30 & $\mathrm{C} 22$ & $\mathrm{C} 23$ & $116.5(3)$ \\
\hline 01 & C7 & $\mathrm{C} 1$ & & $\mathrm{O} 4$ & $\mathrm{C} 22$ & C19 & 117.4(3) \\
\hline 01 & C7 & N1 & & $\mathrm{O} 4$ & C22 & 23 & \\
\hline N1 & C8 & C9 & & $\mathrm{O} 4$ & C22 & C30 & \\
\hline $\mathrm{O} 2$ & C8 & C9 & & $\mathrm{C} 22$ & $\mathrm{C} 23$ & C24 & \\
\hline $\mathrm{O} 2$ & C8 & N1 & & $\mathrm{C} 22$ & $\mathrm{C} 23$ & C26 & 109.5(3) \\
\hline C8 & C9 & $\mathrm{C} 1$ & & $\mathrm{C} 24$ & $\mathrm{C} 23$ & 26 & 111.1 \\
\hline $\mathrm{C} 8$ & C9 & C1 & & $\mathrm{C} 25$ & $\mathrm{C} 24$ & $\mathrm{C} 23$ & $108.8(3)$ \\
\hline C10 & C9 & C1 & & $\mathrm{C} 24$ & $\mathrm{C} 25$ & C9 & $113.9(3)$ \\
\hline & C9 & $\mathrm{C} 8$ & & 03 & $\mathrm{C} 25$ & C9 & \\
\hline & C9 & & & $\mathrm{O} 3$ & $\mathrm{C} 25$ & & \\
\hline $\mathrm{C} 25$ & C9 & C19 & & $\mathrm{C} 27$ & C26 & & $113.4(3)$ \\
\hline C9 & C10 & C16 & & $\mathrm{C} 28$ & C27 & & $116.4(3)$ \\
\hline C11 & C10 & C9 & & $\mathrm{C} 27$ & C28 & & $115.3(3)$ \\
\hline C11 & C10 & C16 & & C30 & C29 & $\mathrm{C} 28$ & $113.2(3)$ \\
\hline C12 & C11 & C10 & & $\mathrm{C} 22$ & C30 & C29 & $119.5(3)$ \\
\hline N1 & C11 & C10 & $104.2(3)$ & $\mathrm{C} 22$ & C30 & C31 & $123.1(3)$ \\
\hline N1 & C11 & C12 & 109.6(3) & C31 & С30 & C29 & $112.3(3)$ \\
\hline C11 & C12 & C13 & $113.6(3)$ & $\mathrm{O} 4$ & C30 & $\mathrm{C} 22$ & $58.9(2)$ \\
\hline
\end{tabular}


Table 5. Bond Angles for 26.

\begin{tabular}{lllllllll} 
Atom Atom Atom & Angle/ $^{\circ}$ & \multicolumn{6}{c}{ Atom Atom Atom Angle/ } \\
\hline C14 & C13 & C12 & $111.1(3)$ & O4 & C30 & C29 & $116.0(3)$ \\
C15 & C13 & C12 & $111.3(3)$ & O4 & C30 & C31 & $116.2(3)$ \\
C15 & C13 & C14 & $110.2(3)$ & C7 & N1 & C11 & $119.2(3)$ \\
C17 & C16 & C10 & $110.3(3)$ & C8 & N1 & C7 & $125.4(3)$ \\
C17 & C16 & C20 & $116.2(3)$ & C8 & N1 & C11 & $113.7(3)$ \\
C20 & C16 & C10 & $112.1(3)$ & C22 & O4 & C30 & $61.3(2)$ \\
C18 & C17 & C16 & $115.5(3)$ & & & &
\end{tabular}

Table 6. Torsion Angles for 26.

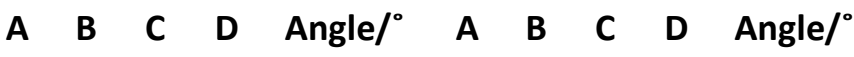
C1 $\quad$ C2 $\quad$ C3 $\quad$ C4 $2.1(5) \quad$ C19 C9 C10 C11 139.1(3)
$\begin{array}{llllllll}\text { C1 } & \text { C7 } & \text { N1 } & \text { C8 } & 41.9(5) & \text { C19 C9 } & \text { C10 C16 } & 15.1(4)\end{array}$
C1 C7 N1 C11 -154.0(3) C19 C9 C25 C24 -51.9(4)
$\begin{array}{lllllllllll}C 2 & \text { C1 } & \text { C6 } & \text { C5 } & -1.6(5) & \text { C19 } & \text { C9 } & \text { C25 } & 03 & 127.0(3)\end{array}$

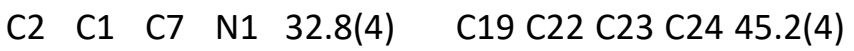

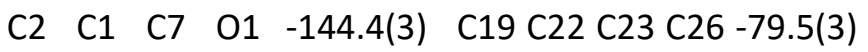
C2 $\quad$ C3 $\quad$ C4 $\quad$ C5 $\quad-2.0(5) \quad$ C19 C22 C30 C29 149.8(3)
C3 C4 C5 C6 $0.2(5) \quad$ C19 C22 C30 C31 -3.1(5)
C4 C5 C6 C1 $\quad 1.7(5) \quad$ C19 C22 C30 O4 $\quad-105.9(3)$
$\begin{array}{llllllllll}\text { C6 } & \text { C1 } & \text { C2 } & \text { C3 } & -0.3(5) & \text { C19 C22 } & 04 & \text { C30 } & 111.5(3)\end{array}$
C6 $\quad$ C1 $\quad$ C7 $\quad$ N1 $\quad-151.8(3) \quad$ C20 C16 C17 C18 177.8(3)
C6 C1 C7 O1 31.1(5) C20 C16 C17 C21 -3.7(5)
C7 C1 C2 C3 $175.2(3) \quad$ C21 C17 C18 C19 -175.7(3)
C7 C1 $\quad$ C6 $\quad$ C5 $\quad-177.2(3) \quad$ C22 C23 C24 C25 $-57.3(4)$
C8 C9 C10 C11 21.5(3) C22 C23 C26 C27 -89.0(3) 
Table 6. Torsion Angles for 26.
$\begin{array}{llllllllll}\text { A } & \text { B } & \text { C } & \text { D } & \text { Angle }^{\circ} & \text { A } & \text { B } & \text { C } & \text { D } & \text { Angle/ } \\ \end{array}$
C8 C9 C10 C16 -102.5(3) C23 C22 C30 C29 2.8(4)
C8 C9 C19 C18 53.1(3) C23 C22 C30 C31 -150.1(3)
C8 C9 C19 C22 $-80.3(3) \quad C 23$ C22 C30 O4 107.1(3)
C8 $\quad$ C9 $\quad 225$ C24 67.6(3) C23 C22 O4 C30 -106.4(3)
C8 $\quad$ C9 $\quad$ C25 O3 $\quad-113.5(3) \quad C 23$ C24 C25 C9 $61.6(4)$

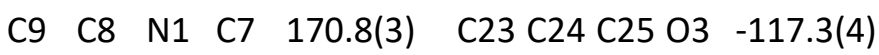
C9 C8 N1 C11 6.0(4) C23 C26 C27 C28 62.2(4)
C9 C10 C11 C12 101.0(3) C24 C23 C26 C27 145.0(3)
C9 C10 C11 N1 $-18.3(3) \quad$ C25 C9 C10 C11 $-94.7(3)$
C9 C10 C16 C17 40.5(4) C25 C9 C10 C16 141.3(3)
C9 C10 C16 C20 171.7(3) C25 C9 C19 C18 171.4(3)
C9 C19 C22 C23 -35.8(4) C25 C9 C19 C22 38.0(4)
C9 C19 C22 C30 176.6(3) C26 C23 C24 C25 66.6(4)
C9 C19 C22 O4 107.0(3) C26 C27 C28 C29 $-55.8(5)$
C10 C9 C19 C18 -61.2(3) C27 C28 C29 C30 78.3(4)
C10 C9 C19 C22 165.4(3) C28 C29 C30 C22 -71.0(4)
C10 C9 C25 C24 -177.9(3) C28 C29 C30 C31 84.6(4)
C10 C9 C25 O3 $0.9(4) \quad$ C28 C29 C30 O4 $-138.4(3)$
C10 C11 C12 C13 72.5(4) C29 C30 O4 C22 110.2(3)
C10 C11 N1 C7 $-157.9(3) \quad$ C30 C22 C23 C24 -165.7(3)
C10 C11 N1 C8 8.0(4) C30 C22 C23 C26 69.6(4)
C10 C16 C17 C18 -53.2(4) C31 C30 O4 C22 -114.5(3)
C10 C16 C17 C21 125.3(4) N1 C8 C9 C10 -17.3(3)
C11 C10 C16 C17-78.1(4) N1 C8 C9 C19-135.9(3)
C11 C10 C16 C20 53.1(4) N1 C8 C9 C25 103.3(3) 
Table 6. Torsion Angles for 26.

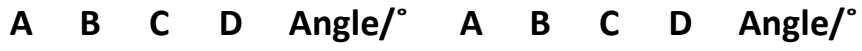
C11 C12 C13 C14 -161.4(3) N1 C11 C12 C13 -171.4(3)
C11 C12 C13 C15 75.4(4) O1 C7 N1 C8 $\quad-140.8(3)$
$\begin{array}{llllllll}\text { C12 C11 N1 C7 } & 80.1(4) & \text { O1 } & \text { C7 } & \text { N1 } & \text { C11 23.3(5) }\end{array}$

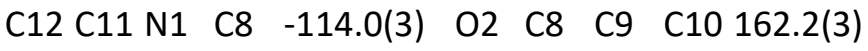
C16 C10 C11 C12 -137.1(3) O2 C8 C9 C19 43.5(4)
C16 C10 C11 N1 $103.6(3) \quad 02 \quad$ C8 C9 C25 $\quad-77.2(4)$

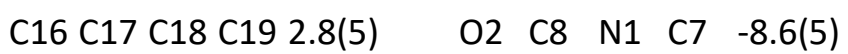
C17 C18 C19 C9 54.1(4) O2 C8 N1 C11 $-173.5(3)$
C17 C18 C19 C22 -173.9(3) O4 C22 C23 C24 -97.9(3)
C18 C19 C22 C23 -162.2(3) O4 C22 C23 C26 137.4(3)
C18 C19 C22 C30 50.2(4) O4 C22 C30 C29 -104.3(3)
C18 C19 C22 O4 $-19.3(4) \quad 04 \quad$ C22 C30 C31 102.8(3)

Table 7. Hydrogen Atom Coordinates $\left(\AA \times 10^{4}\right)$ and Isotropic Displacement Parameters $\left(\AA^{2} \times 10^{3}\right)$ for 26.

\begin{tabular}{lllll} 
Atom & $\boldsymbol{x}$ & $\boldsymbol{y}$ & $\boldsymbol{z}$ & $\mathrm{U}(\mathrm{eq})$ \\
\hline H2 & 234.22 & 5691.2 & 5894.72 & 36 \\
H3 & -1314.61 & 5994.93 & 5240.19 & 42 \\
H4 & -1141.13 & 7871.59 & 4803.93 & 43 \\
H5 & 461.8 & 9493.3 & 5044.42 & 42 \\
H6 & 1919.6 & 9238.21 & 5724.02 & 38 \\
H10 & 3686.31 & 4050.57 & 7179.61 & 32 \\
H11 & 3447.48 & 6581.46 & 7097.5 & 31 \\
H12A & 1036.52 & 4748.92 & 7084.78 & 36 \\
H12B & 729.07 & 6242.42 & 7128.77 & 36 \\
H13 & 2542.83 & 4959.36 & 7815.78 & 39
\end{tabular}


Table 7. Hydrogen Atom Coordinates $\left(\AA \times 10^{4}\right)$ and Isotropic Displacement Parameters $\left(\AA^{2} \times 10^{3}\right)$ for 26.

\begin{tabular}{|c|c|c|c|c|}
\hline Atom & $x$ & $y$ & $z$ & $U(e q)$ \\
\hline $\mathrm{H} 14 \mathrm{~A}$ & -638.81 & 5258.3 & 7889.43 & 71 \\
\hline H14B & 415.99 & 4743.61 & 8305.42 & 71 \\
\hline $\mathrm{H} 14 \mathrm{C}$ & 214.89 & 3926.96 & 7832.97 & 71 \\
\hline $\mathrm{H} 15 \mathrm{~A}$ & 2666.68 & 7201.38 & 7819.73 & 75 \\
\hline H15B & 1983.52 & 6676.73 & 8304.7 & 75 \\
\hline $\mathrm{H} 15 \mathrm{C}$ & 892.89 & 7270.22 & 7912.97 & 75 \\
\hline H16 & 6231.42 & 4213.77 & 7098.44 & 48 \\
\hline $\mathrm{H} 18$ & 6767.1 & 5195.95 & 5930.29 & 40 \\
\hline H19 & 5913.96 & 3194.64 & 6494.96 & 33 \\
\hline $\mathrm{H} 2 \mathrm{AA}$ & 5456.14 & 6650.05 & 7463.84 & 90 \\
\hline $\mathrm{H} 2 \mathrm{OB}$ & 6939.24 & 5855.77 & 7588.91 & 90 \\
\hline $\mathrm{H} 2 \mathrm{OC}$ & 5327.18 & 5357.66 & 7759.41 & 90 \\
\hline $\mathrm{H} 21 \mathrm{~A}$ & 7609.19 & 7082.5 & 6317.34 & 86 \\
\hline $\mathrm{H} 21 \mathrm{~B}$ & 7979.72 & 6879.19 & 6864.92 & 86 \\
\hline $\mathrm{H} 21 \mathrm{C}$ & 6437.47 & 7544.87 & 6710.36 & 86 \\
\hline $\mathrm{H} 23$ & 4049.4 & 1510.08 & 5476.36 & 35 \\
\hline $\mathrm{H} 24 \mathrm{~A}$ & 2291.04 & 3056.72 & 5707.5 & 38 \\
\hline $\mathrm{H} 24 \mathrm{~B}$ & 1832.3 & 1730.91 & 5948.69 & 38 \\
\hline $\mathrm{H} 26 \mathrm{~A}$ & 5185.19 & 1050.84 & 6392.9 & 38 \\
\hline $\mathrm{H} 26 \mathrm{~B}$ & 3632.97 & 363.5 & 6265.11 & 38 \\
\hline $\mathrm{H} 27 \mathrm{~A}$ & 5585.49 & -1029.64 & 6100.51 & 50 \\
\hline $\mathrm{H} 27 \mathrm{~B}$ & 4786.41 & -650.14 & 5616.47 & 50 \\
\hline $\mathrm{H} 28 \mathrm{~A}$ & 7363.23 & -706.18 & 5502.58 & 54 \\
\hline $\mathrm{H} 28 \mathrm{~B}$ & 7633.88 & 253.35 & 5931.01 & 54 \\
\hline $\mathrm{H} 29 \mathrm{~A}$ & 7837.96 & 1137.03 & 5128.57 & 47 \\
\hline
\end{tabular}


Table 7. Hydrogen Atom Coordinates $\left(\AA \times 10^{4}\right)$ and Isotropic Displacement Parameters $\left(\AA^{2} \times 10^{3}\right)$ for 26.

\begin{tabular}{lllll} 
Atom & $\boldsymbol{x}$ & $\boldsymbol{y}$ & $\boldsymbol{z}$ & $\mathrm{U}(\mathbf{e q})$ \\
\hline H29B & 6041.42 & 1068.41 & 5111.42 & 47 \\
H31A & 8920.95 & 3184.21 & 5436.85 & 59 \\
H31B & 8894.24 & 2255.57 & 5884.29 & 59 \\
H31C & 8224.1 & 3663.65 & 5926.33 & 59
\end{tabular}




\section{Copies of NMR spectra}

(1S,2R)-1,2-Dihydroxy-1-methylcycloheptane (13)

${ }^{1} \mathrm{H} \mathrm{NMR}\left(\mathrm{CDCl}_{3}, 400 \mathrm{MHz}\right)$

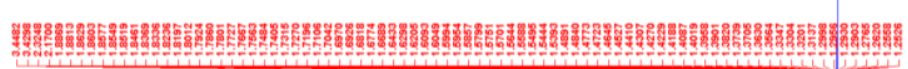

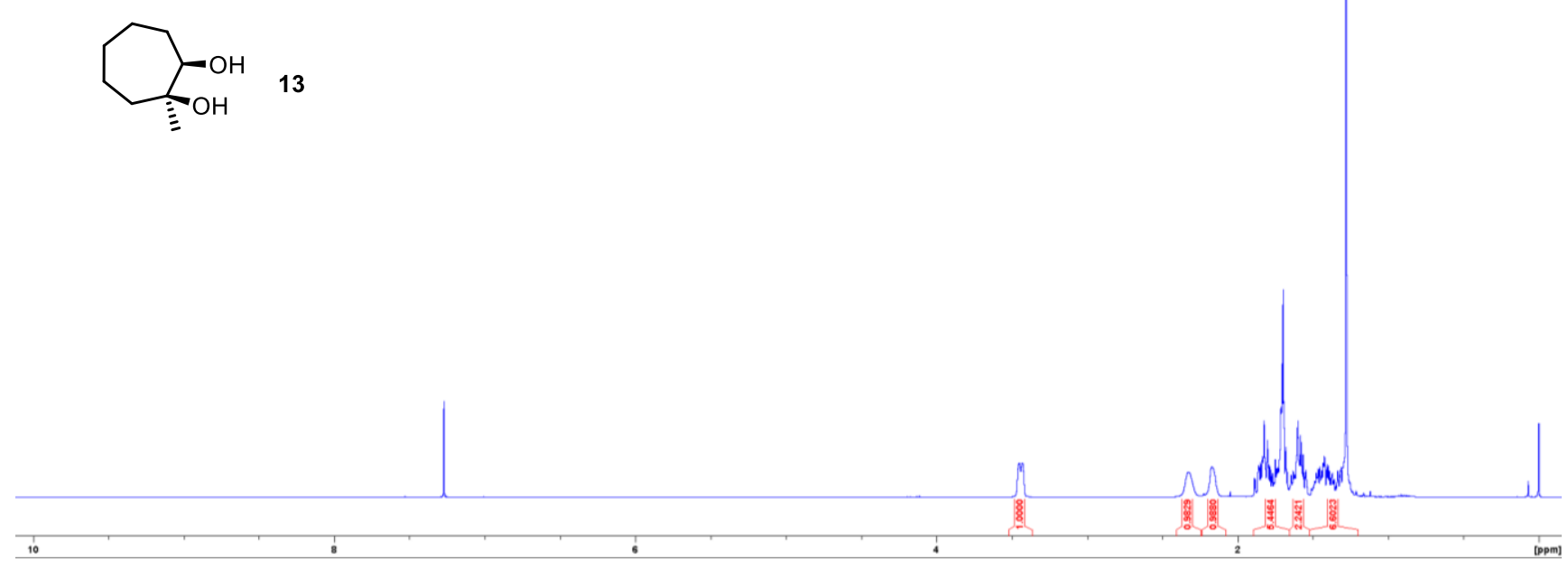

${ }^{13} \mathrm{C} \mathrm{NMR}\left(\mathrm{CDCl}_{3}, 100 \mathrm{MHz}\right)$

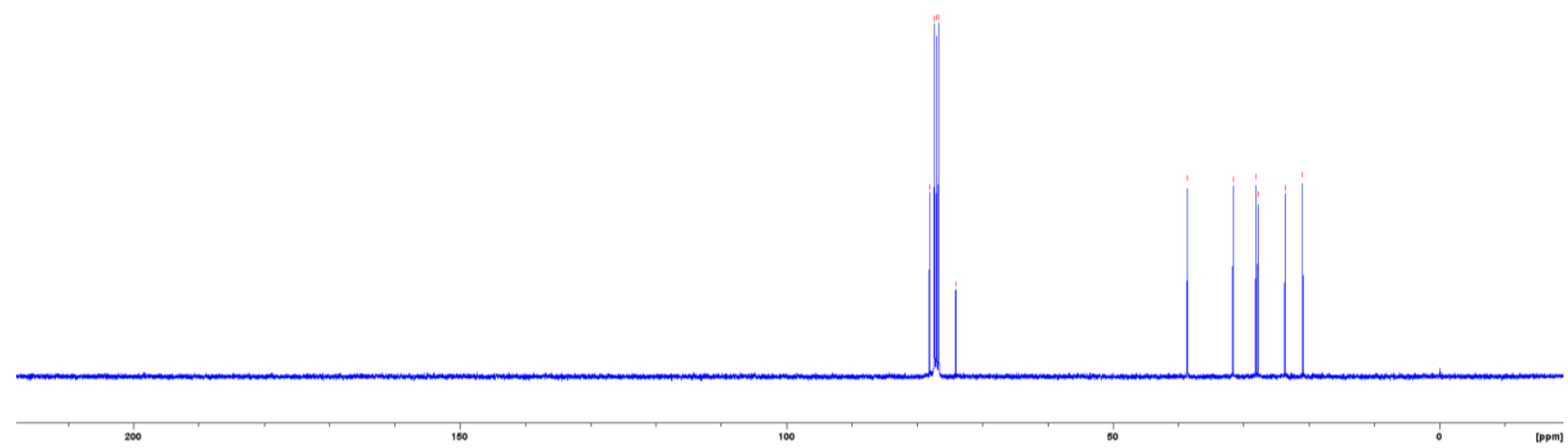


(2S)-2-Hydroxy-2-methylcycloheptan-1-one (S1)

${ }^{1} \mathrm{H}$ NMR $\left(\mathrm{CDCl}_{3}, 400 \mathrm{MHz}\right)$

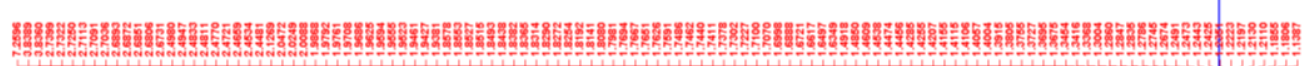

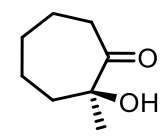

s1

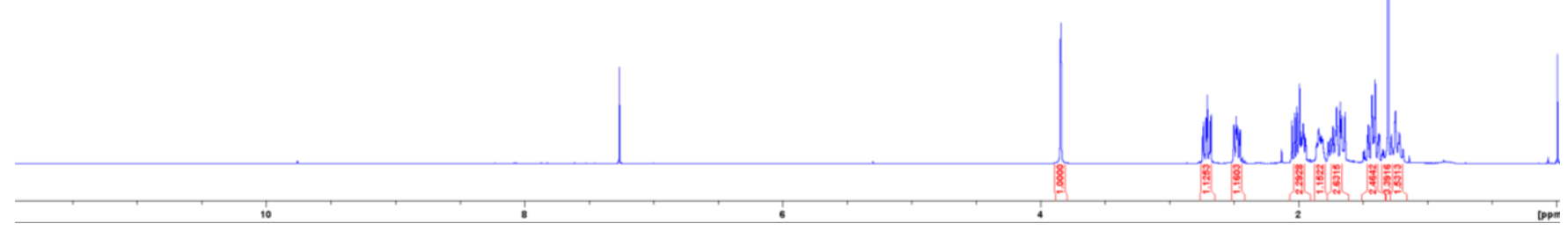

${ }^{13} \mathrm{C} \mathrm{NMR}\left(\mathrm{CDCl}_{3}, 100 \mathrm{MHz}\right)$
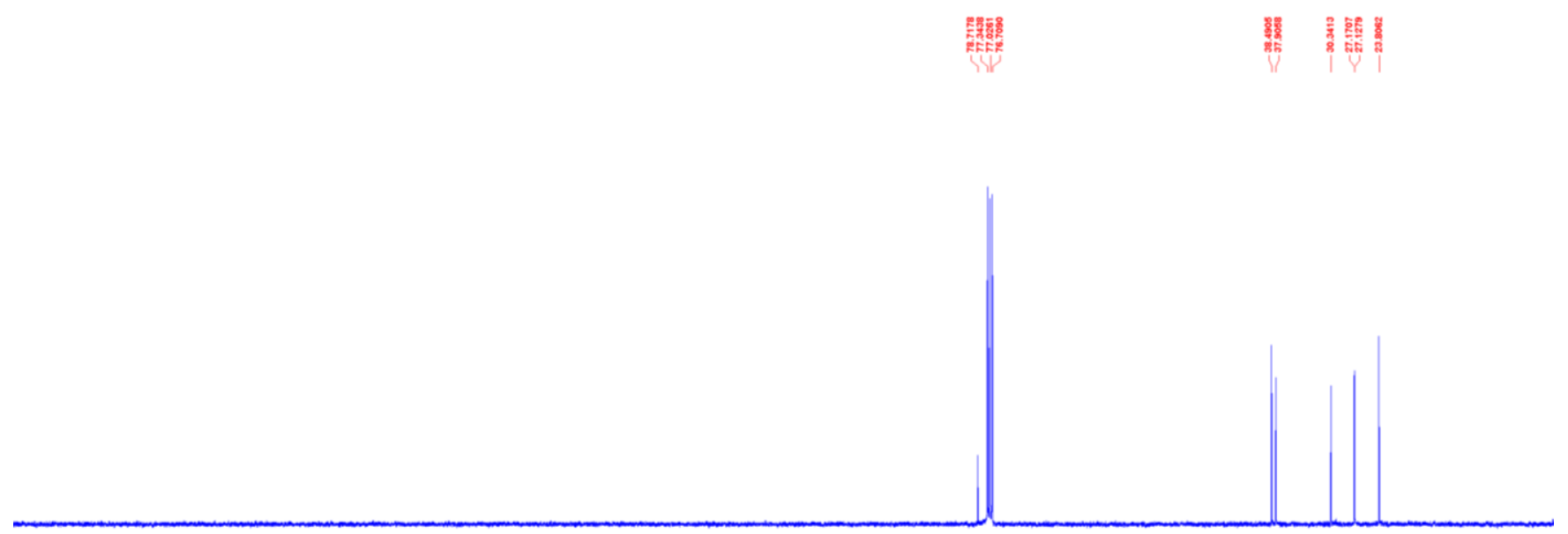
(2S)-2-Trimethylsilyloxy-2-methylcycloheptan-1-one (15)

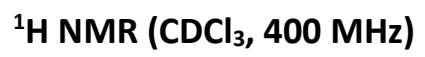

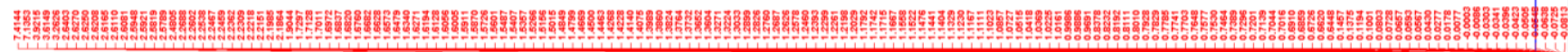

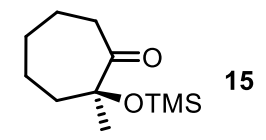

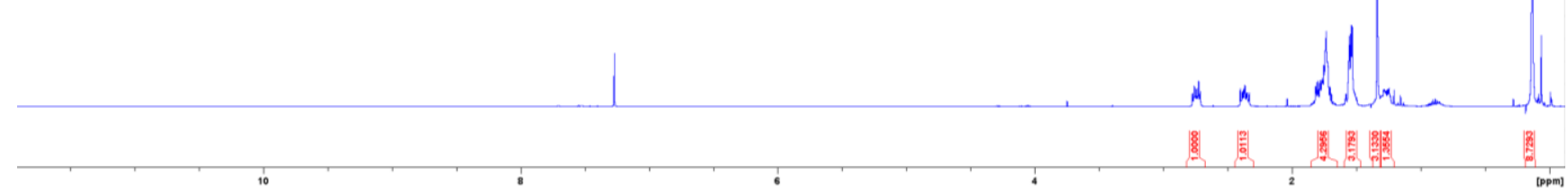

${ }^{13} \mathrm{C} \mathrm{NMR}\left(\mathrm{CDCl}_{3}, 100 \mathrm{MHz}\right)$

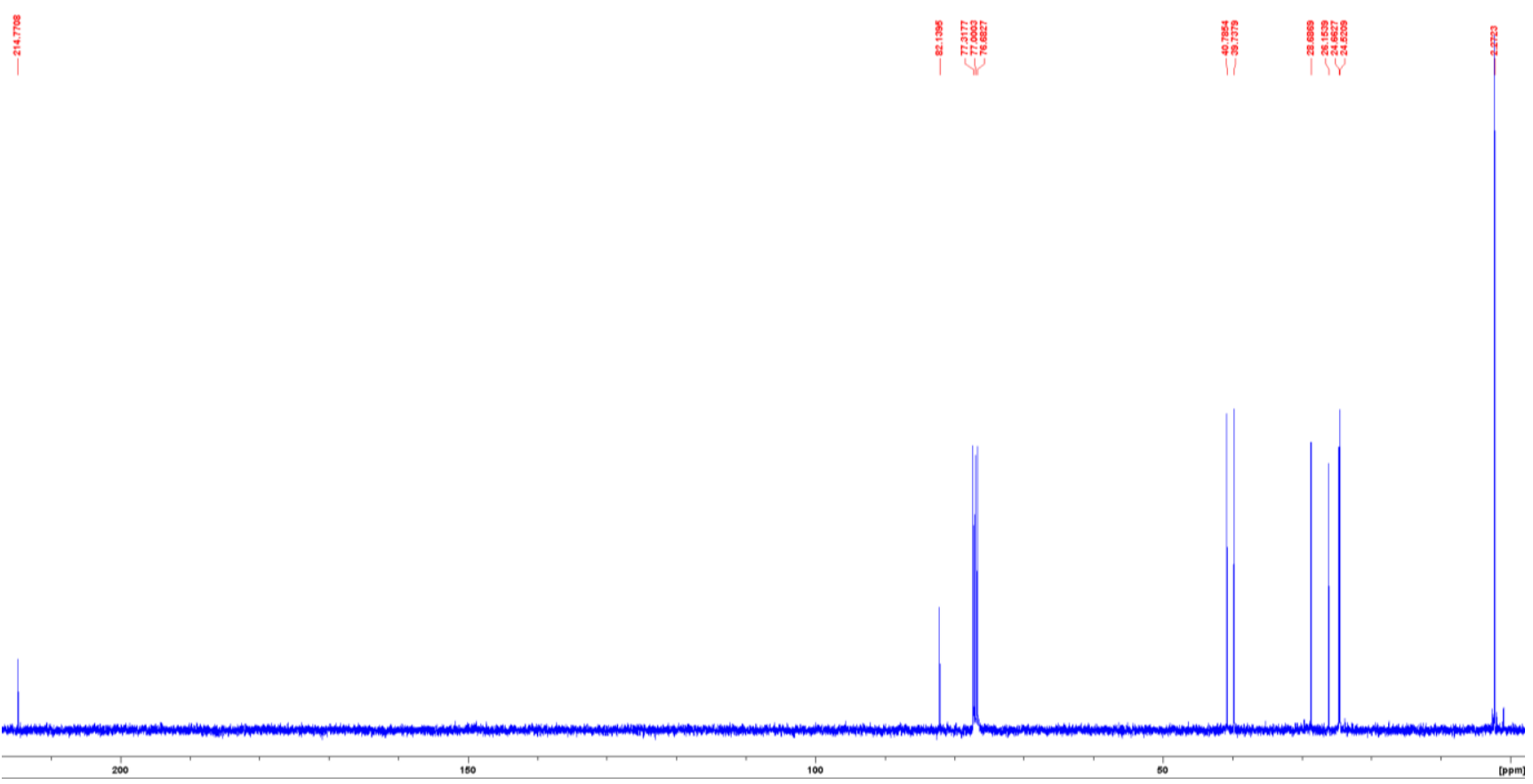


(7S)-7-Hydroxy-7-methyl-1-trifluoromethanesulfonyloxycyclohept-1-ene (12)

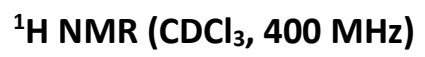

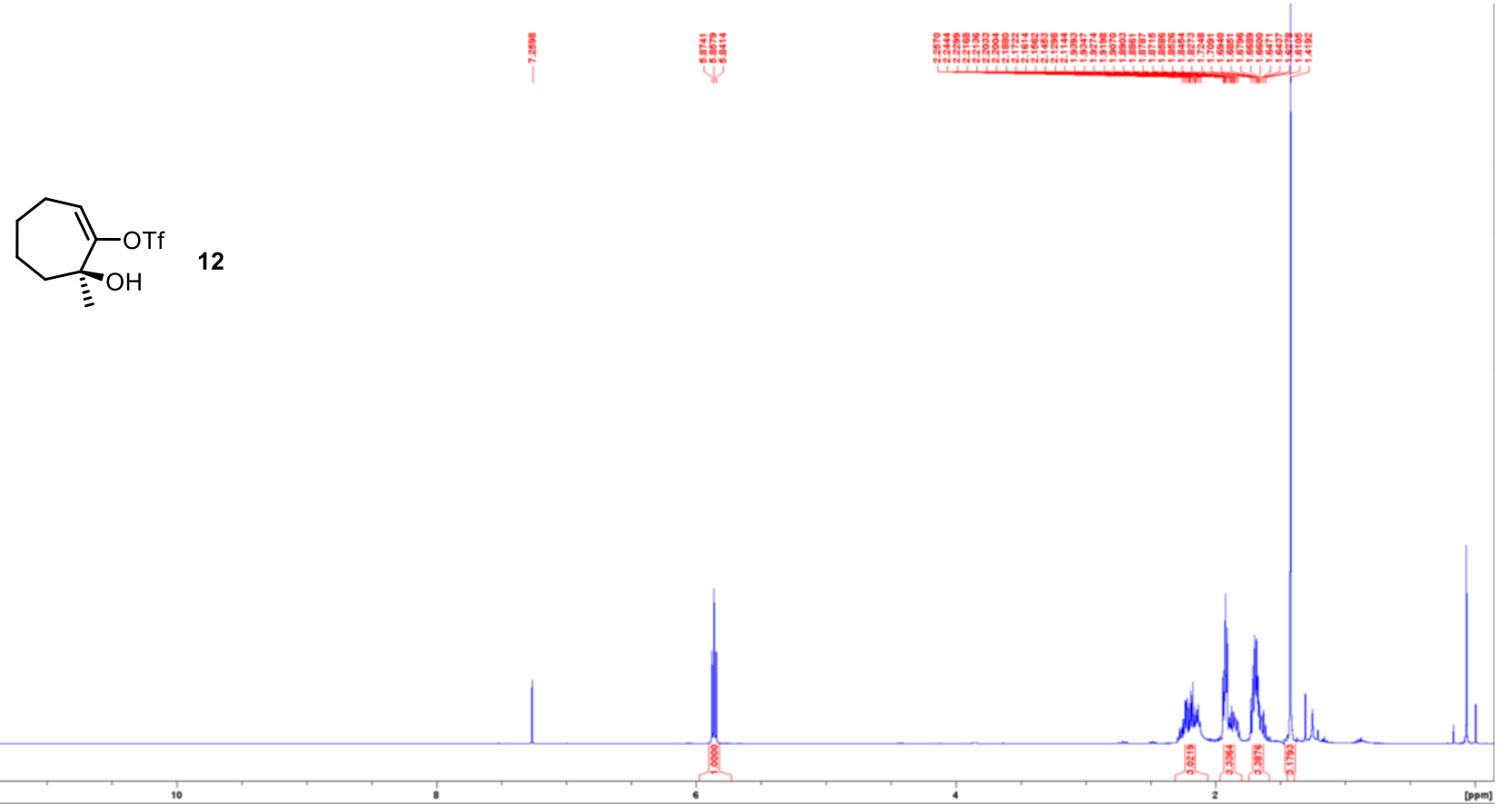

${ }^{13} \mathrm{C} \mathrm{NMR}\left(\mathrm{CDCl}_{3}, 100 \mathrm{MHz}\right)$

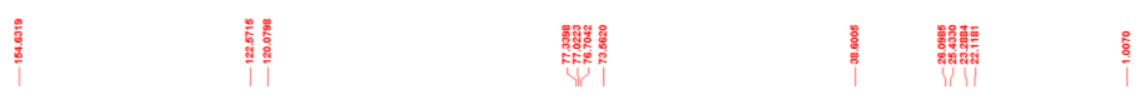

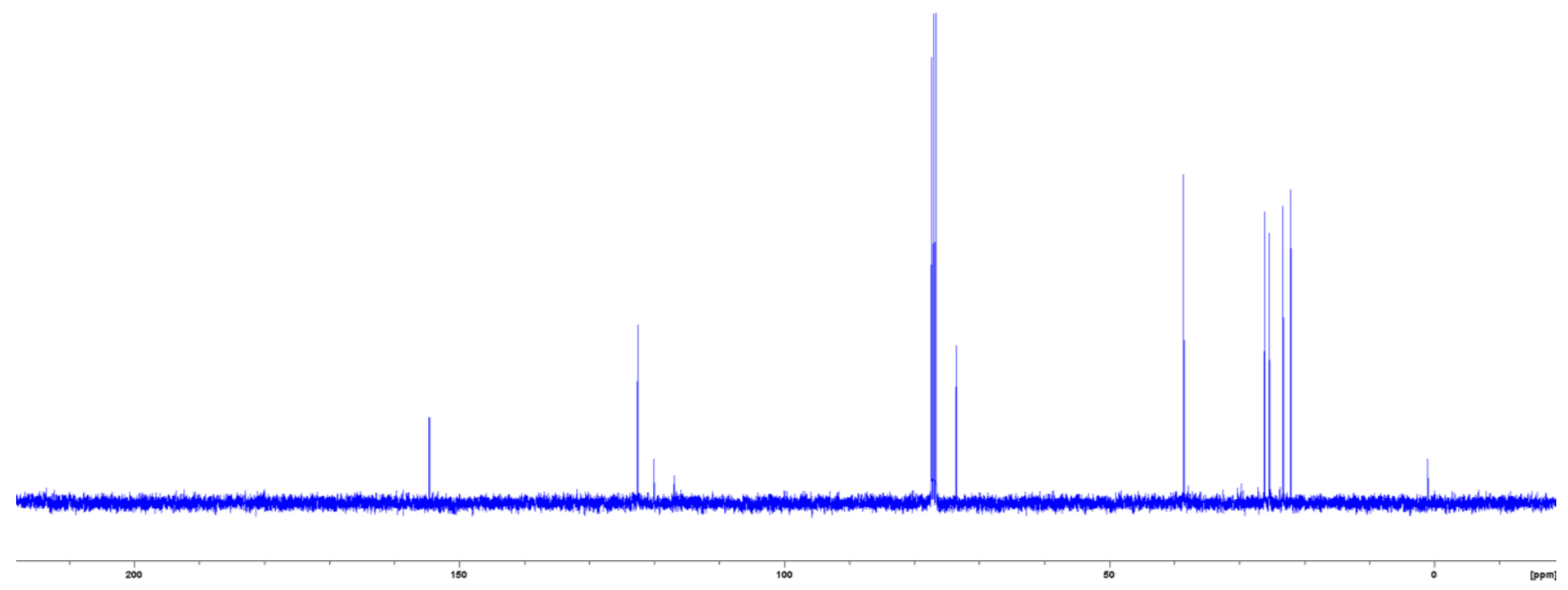


(7S)-7-Acetoxy-7-methyl-1-trifluoromethanesulfonyloxycyclohept-1-ene (16)

${ }^{1} \mathrm{H}$ NMR $\left(\mathrm{CDCl}_{3}, 400 \mathrm{MHz}\right)$

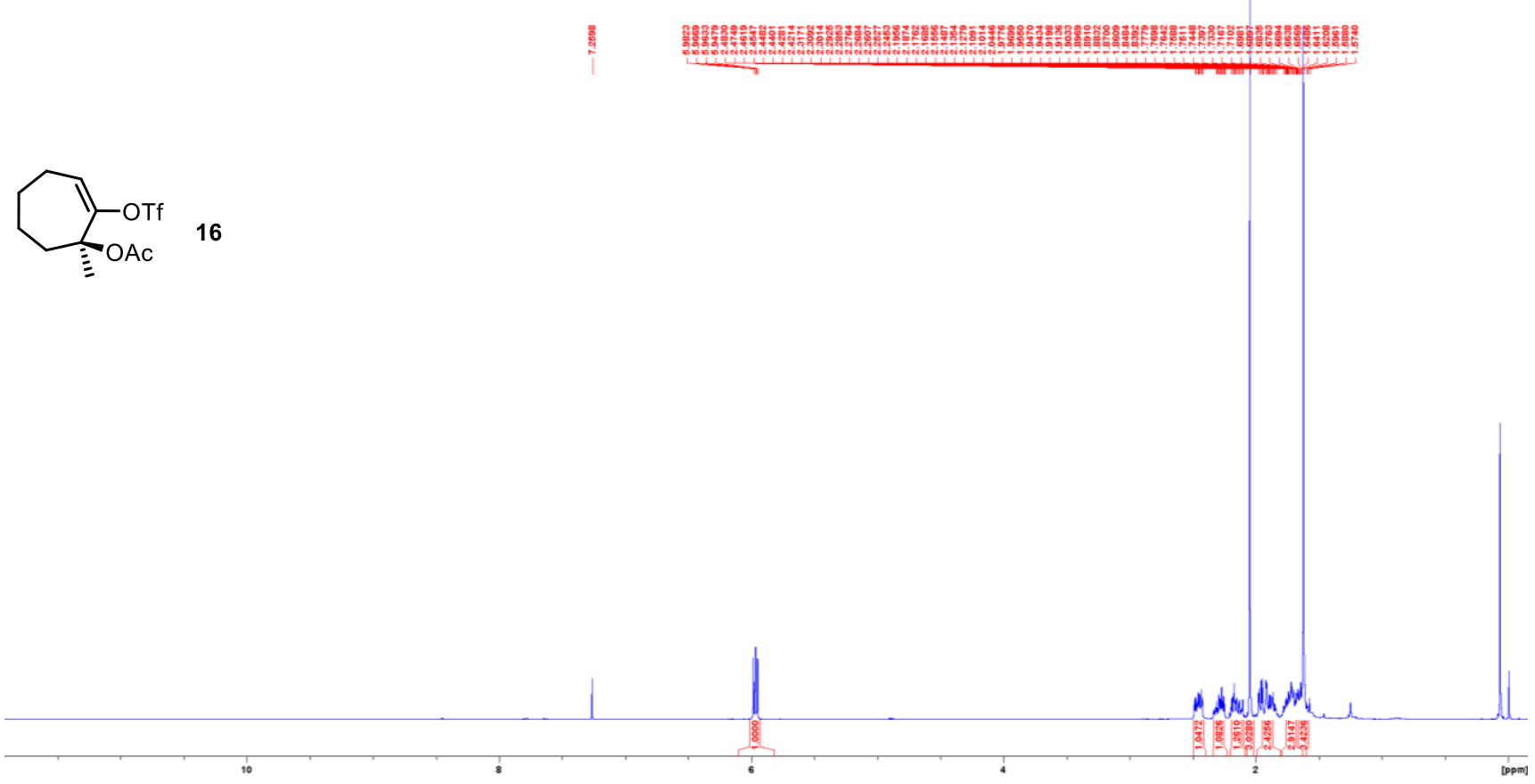

$\left.{ }^{13} \mathrm{C} \mathrm{NMR} \mathrm{(CDCl}{ }_{3}, 100 \mathrm{MHz}\right)$

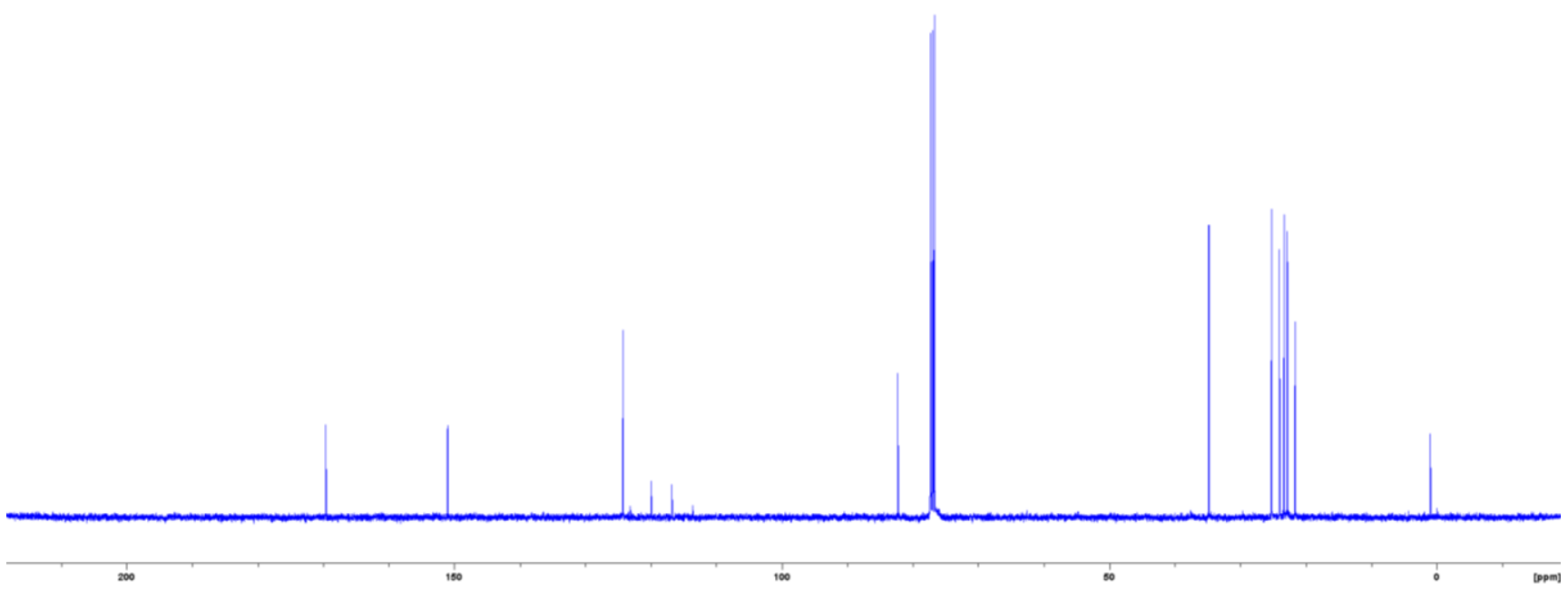


(7S)- 7-Acetoxy-7-methyl-1-[(1E,3E)-3-methylpenta-1,3-dien-1-yl]cyclohept-1-ene (10), as a 4:1 mixture with the $1 Z$ isomer

${ }^{1} \mathrm{H} \mathrm{NMR}\left(\mathrm{CDCl}_{3}, 400 \mathrm{MHz}\right)$

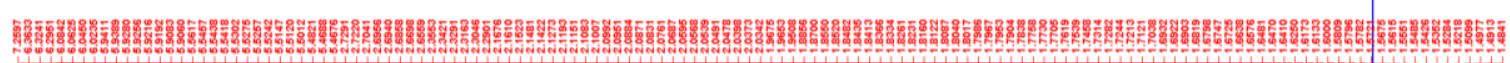

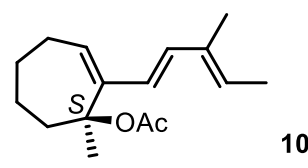

10

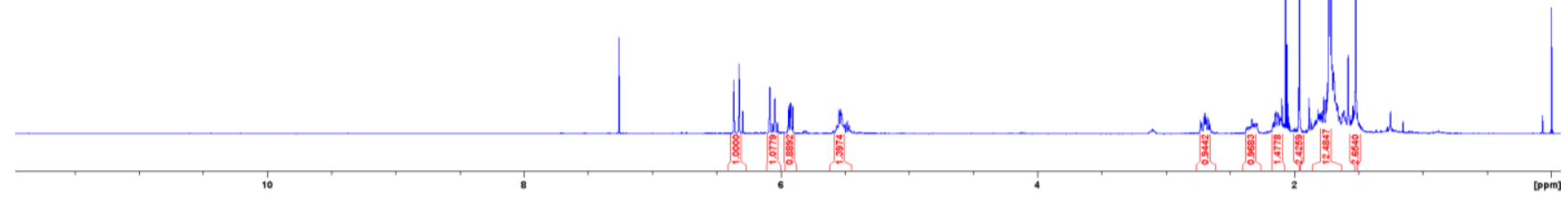

${ }^{13} \mathrm{C} \mathrm{NMR}\left(\mathrm{CDCl}_{3}, 100 \mathrm{MHz}\right)$
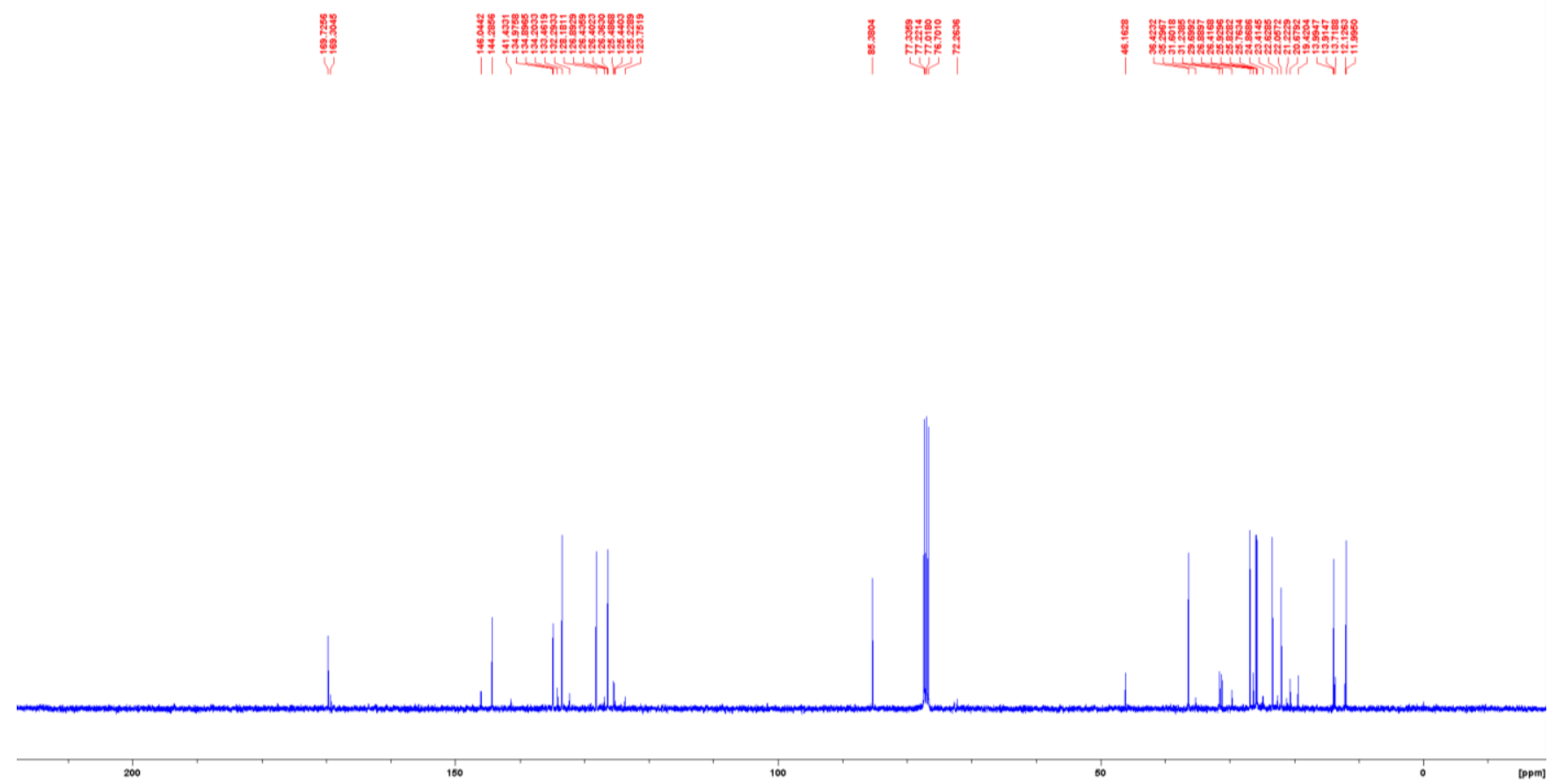
(rac)-2-[3-Methyl-2-vinylcyclohept-2-en-1-yl]acetic acid (S2)

${ }^{1} \mathrm{H} \mathrm{NMR}\left(\mathrm{CDCl}_{3}, 600 \mathrm{MHz}\right)$

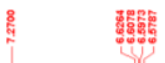

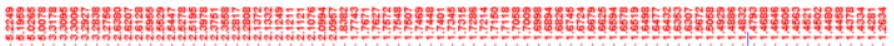

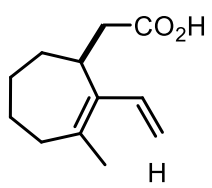

s2

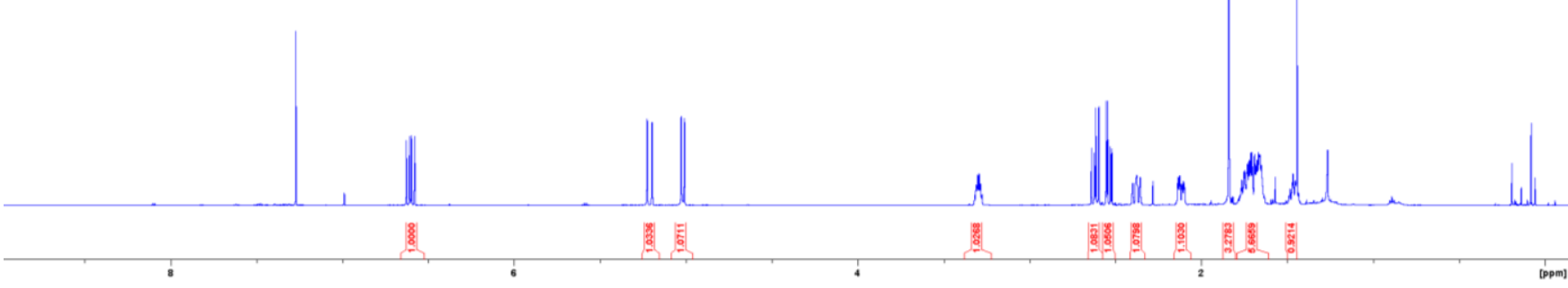

${ }^{13} \mathrm{C} \mathrm{NMR} \mathrm{(CDCl}, 100 \mathrm{MHz}$, DEPTQ experiment)

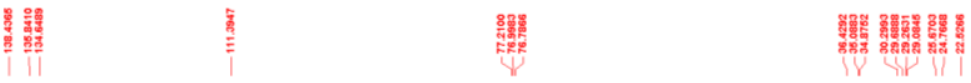

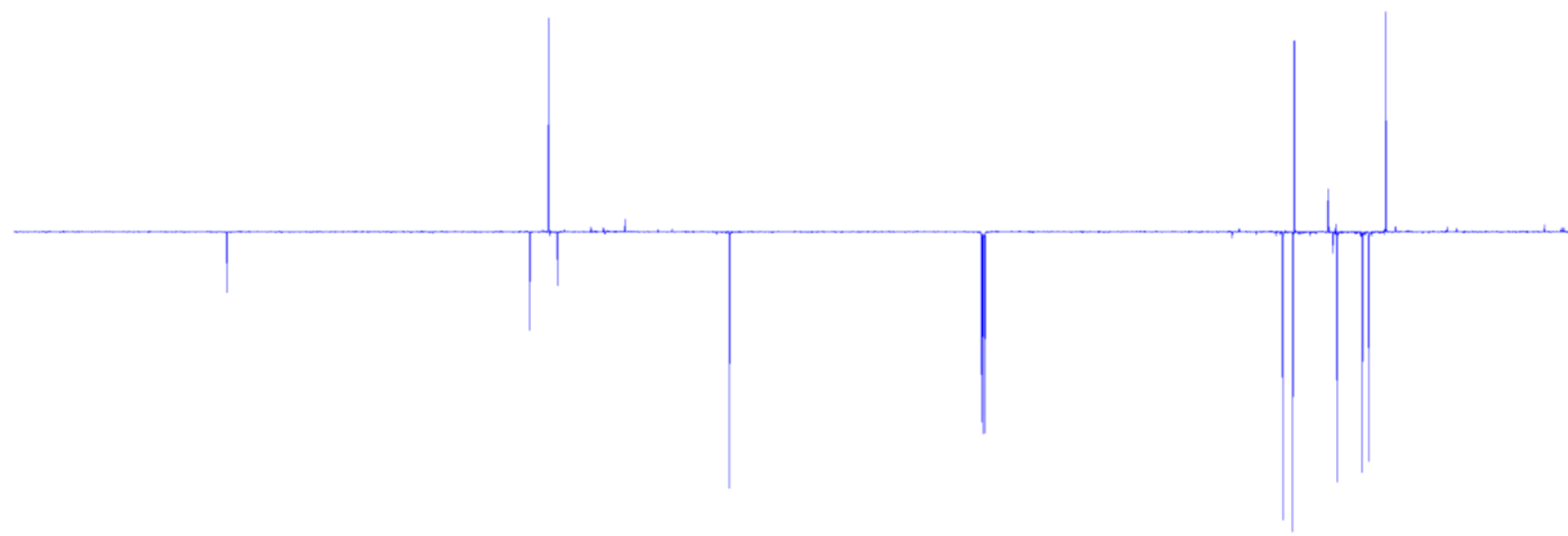


(rac)-2-[3-Methyl-2-vinylcyclohept-2-en-1-yl]acetic acid, methyl ester (19)

${ }^{1} \mathrm{H} \mathrm{NMR}\left(\mathrm{CDCl}_{3}, 600 \mathrm{MHz}\right)$

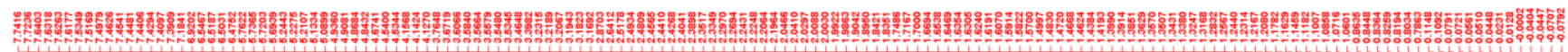

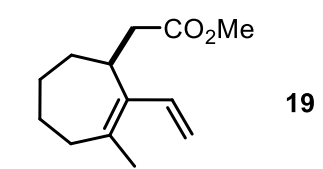

9

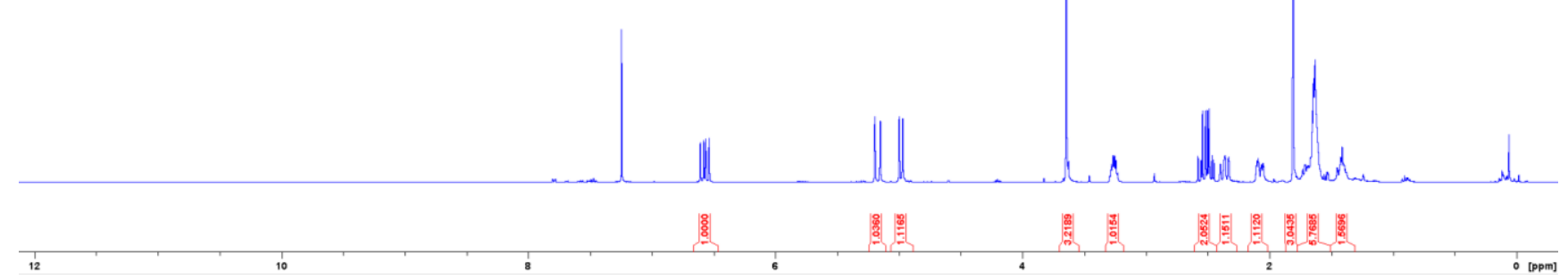

${ }^{13} \mathrm{C} \mathrm{NMR}\left(\mathrm{CDCl}_{3}, 100 \mathrm{MHz}\right.$, DEPTQ experiment)

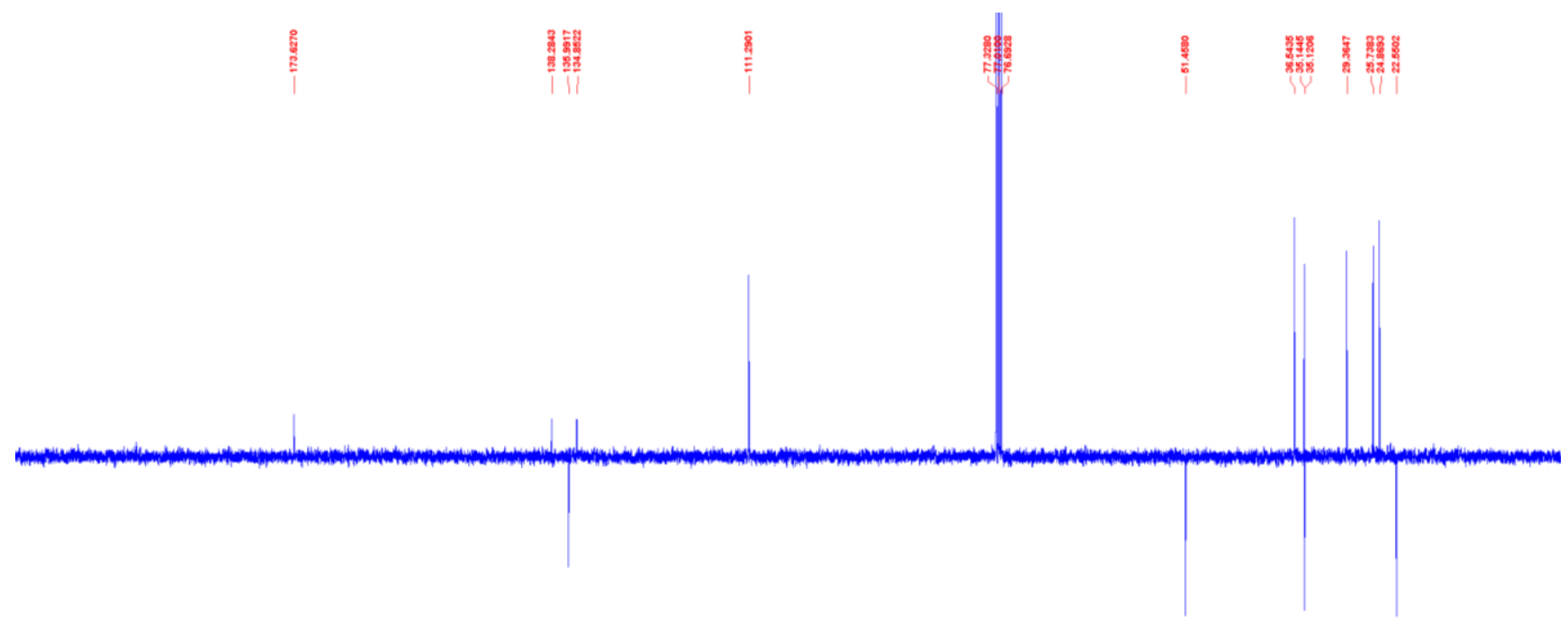


(E)-2-(3-Methyl-2-(2-(4,4,5,5-tetramethyl-1,3,2-dioxaborolan-2-yl)vinyl)cyclohept-2-en-1-yl)acetic acid, methyl ester (20)

${ }^{1} \mathrm{H} \mathrm{NMR}\left(\mathrm{CDCl}_{3}, 400 \mathrm{MHz}\right)$

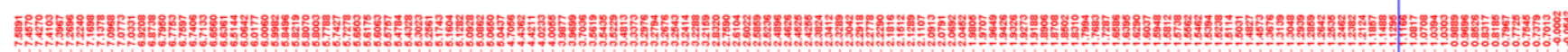

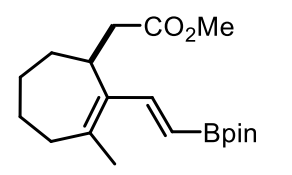

20

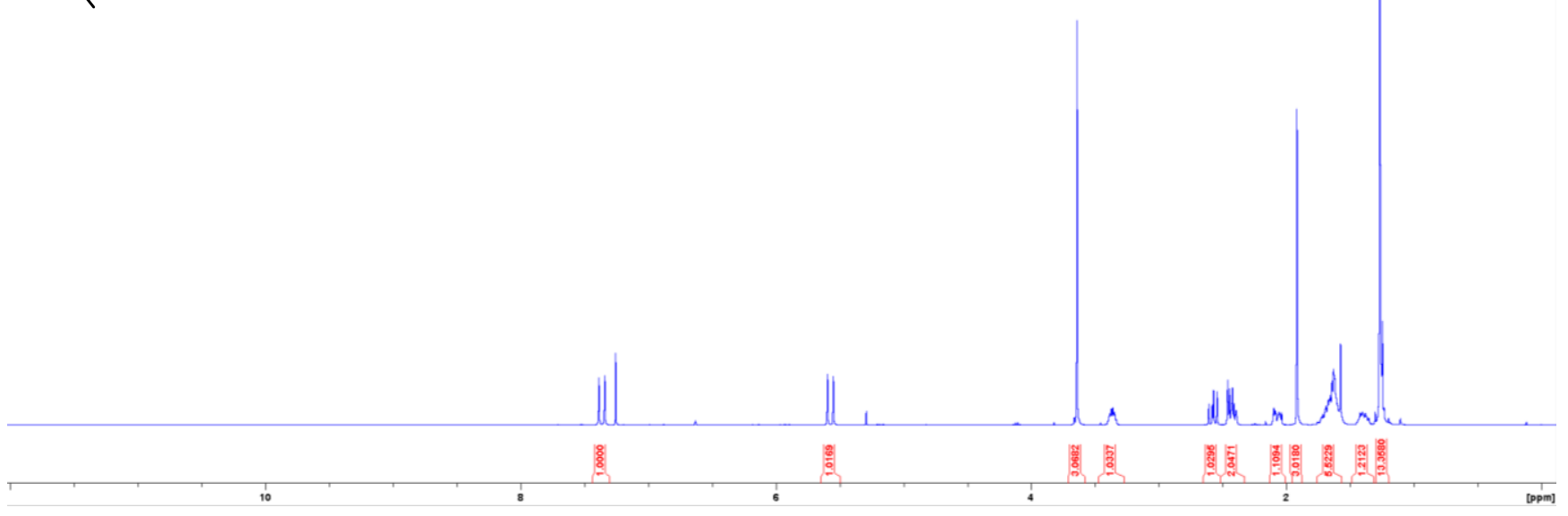

${ }^{13} \mathrm{C}$ NMR $\left(\mathrm{CDCl}_{3}, 100 \mathrm{MHz}\right.$, DEPTQ experiment)

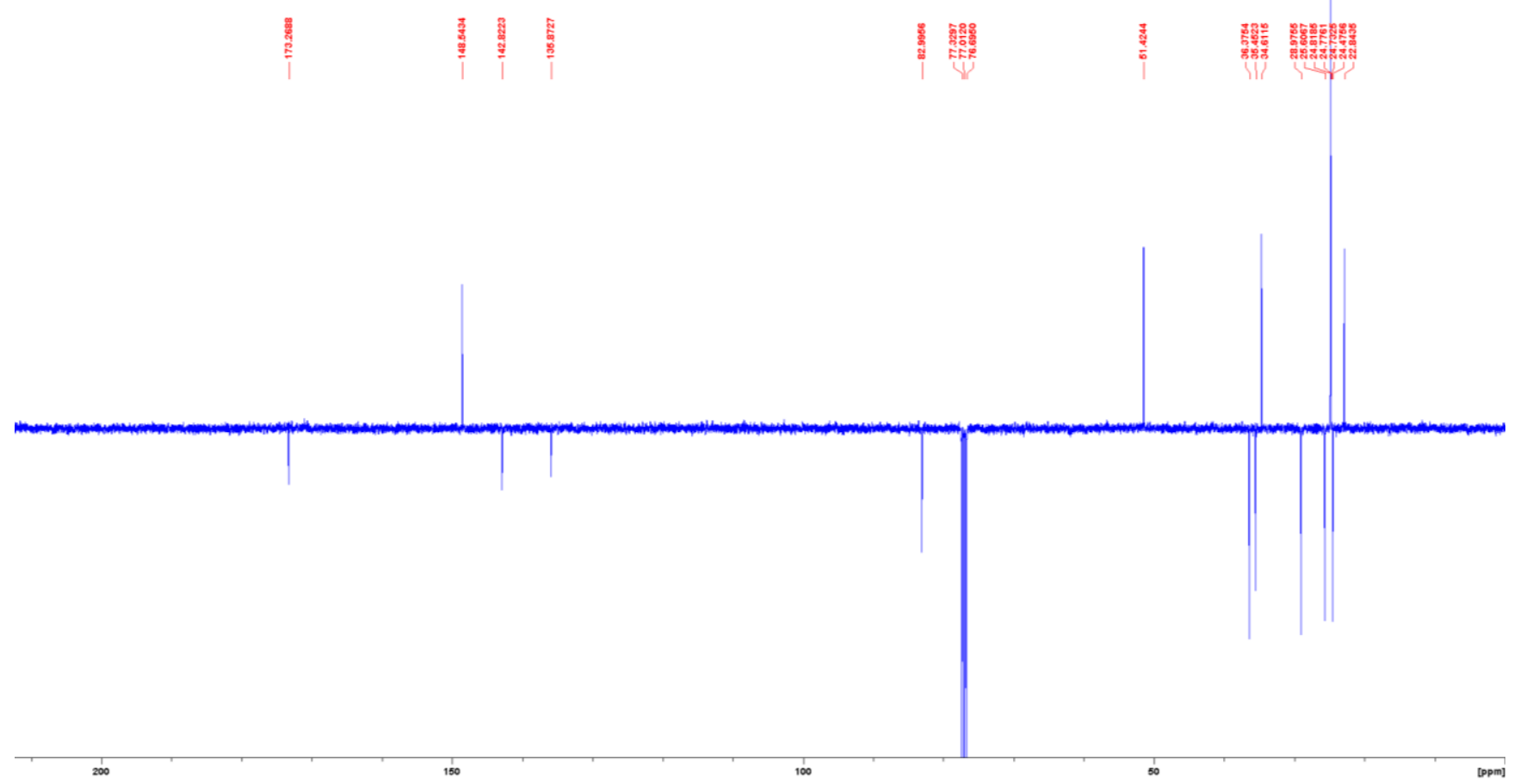


(E)-2-lodobut-2-ene (21)

${ }^{1} \mathrm{H}$ NMR $\left(\mathrm{CDCl}_{3}, 400 \mathrm{MHz}\right)$

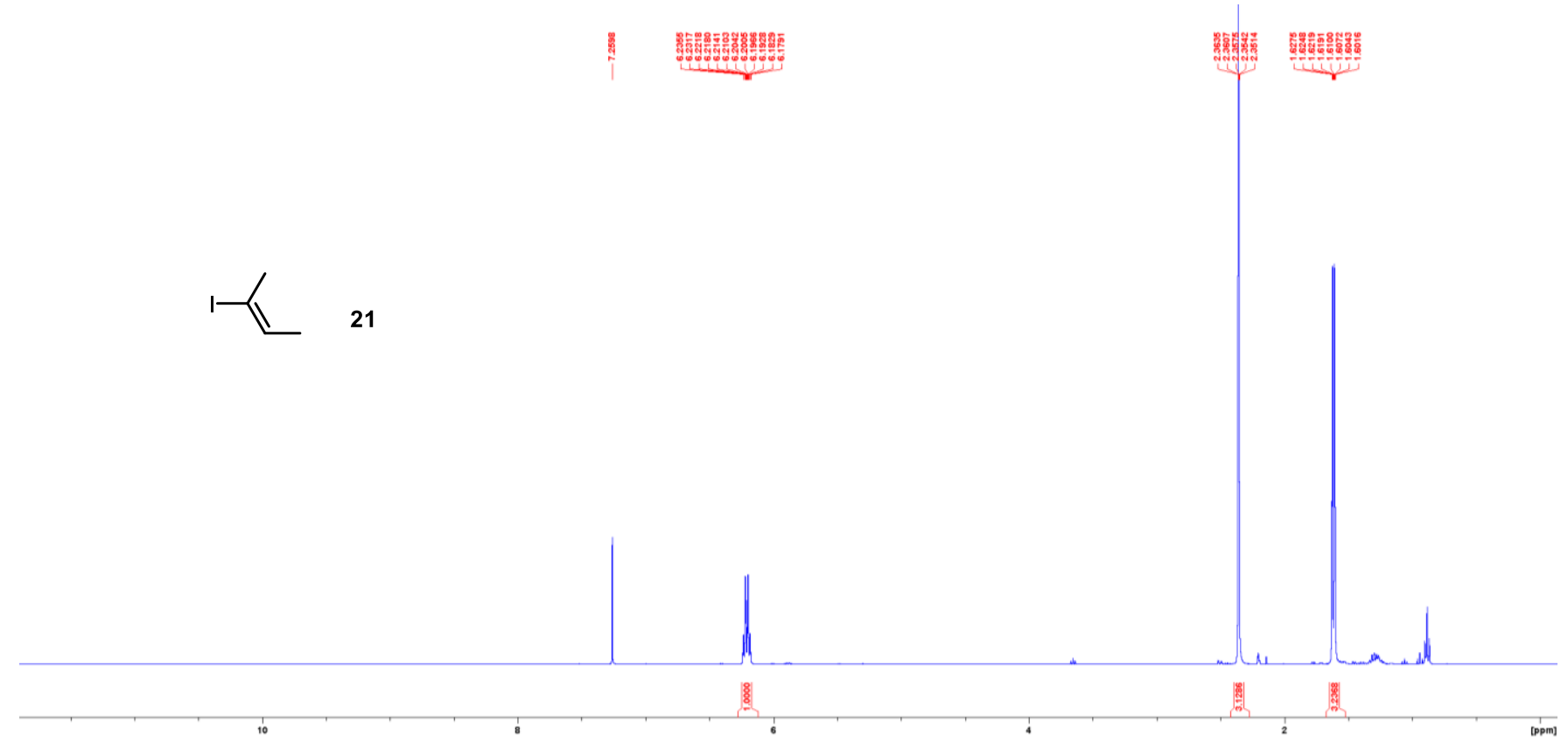

${ }^{13} \mathrm{C} \mathrm{NMR}\left(\mathrm{CDCl}_{3}, 100 \mathrm{MHz}\right.$, DEPTQ experiment)

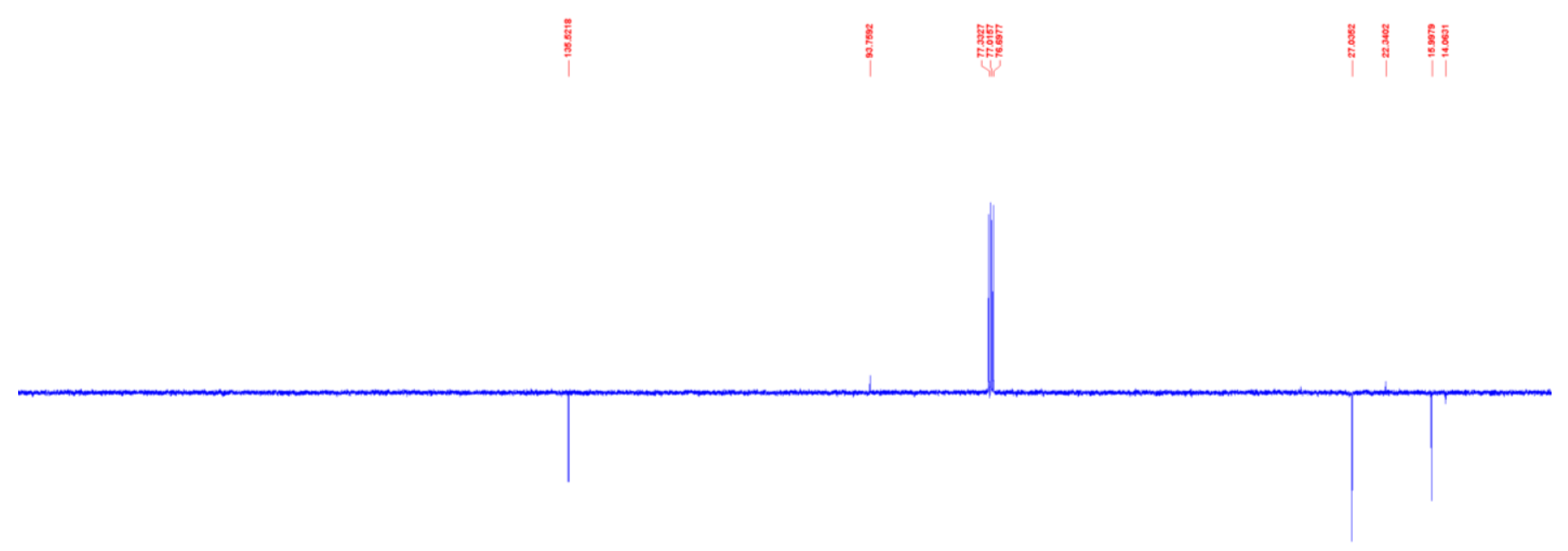


2-(3-Methyl-2-((1E,3E)-3-methylpenta-1,3-dien-1-yl)cyclohept-2-en-1-yl)acetic acid, methyl ester (S3)

${ }^{1} \mathrm{H}$ NMR $\left(\mathrm{CDCl}_{3}, 600 \mathrm{MHz}\right)$

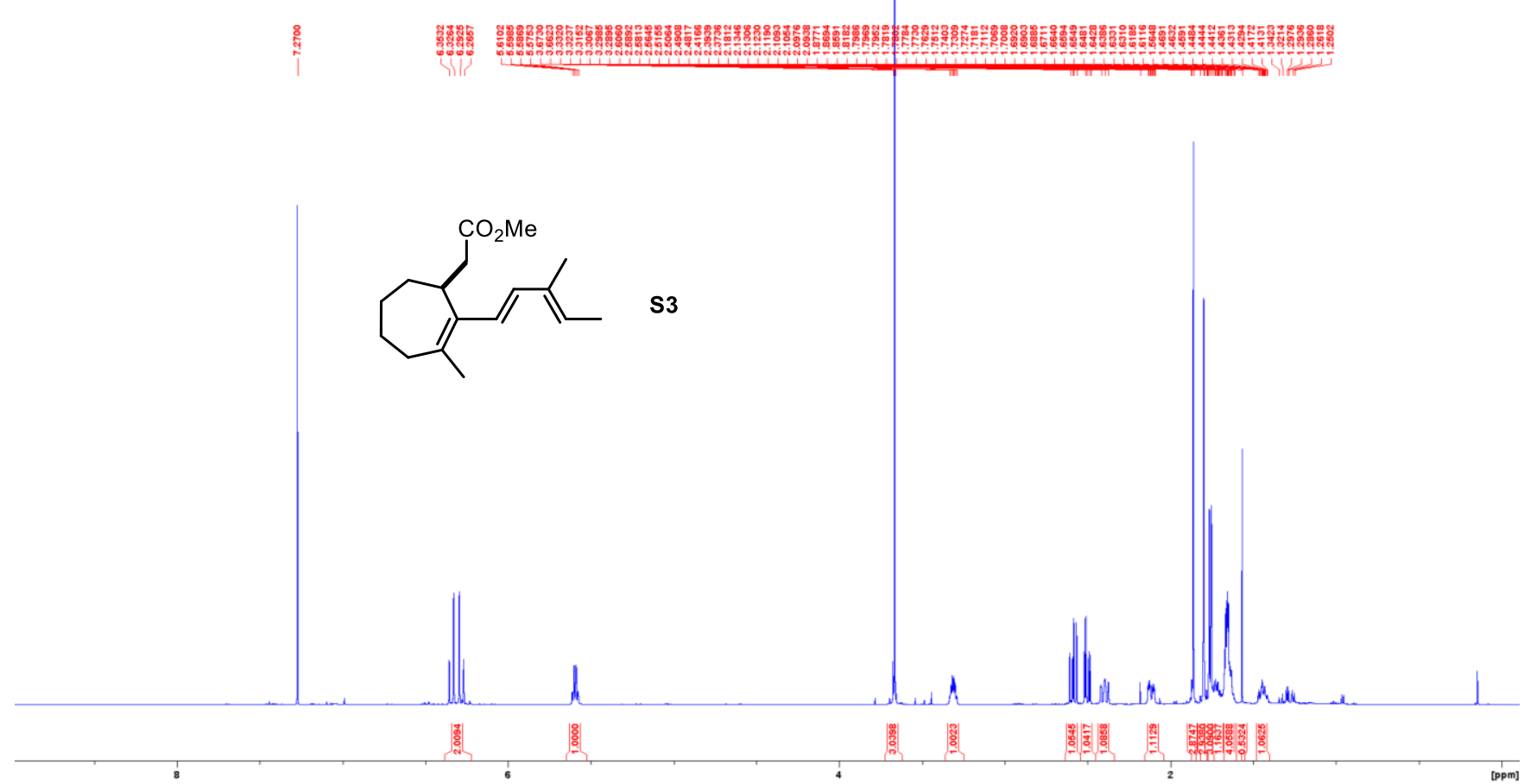

${ }^{13} \mathrm{C} \mathrm{NMR} \mathrm{(CDCl}, 150 \mathrm{MHz}$, DEPTQ experiment)

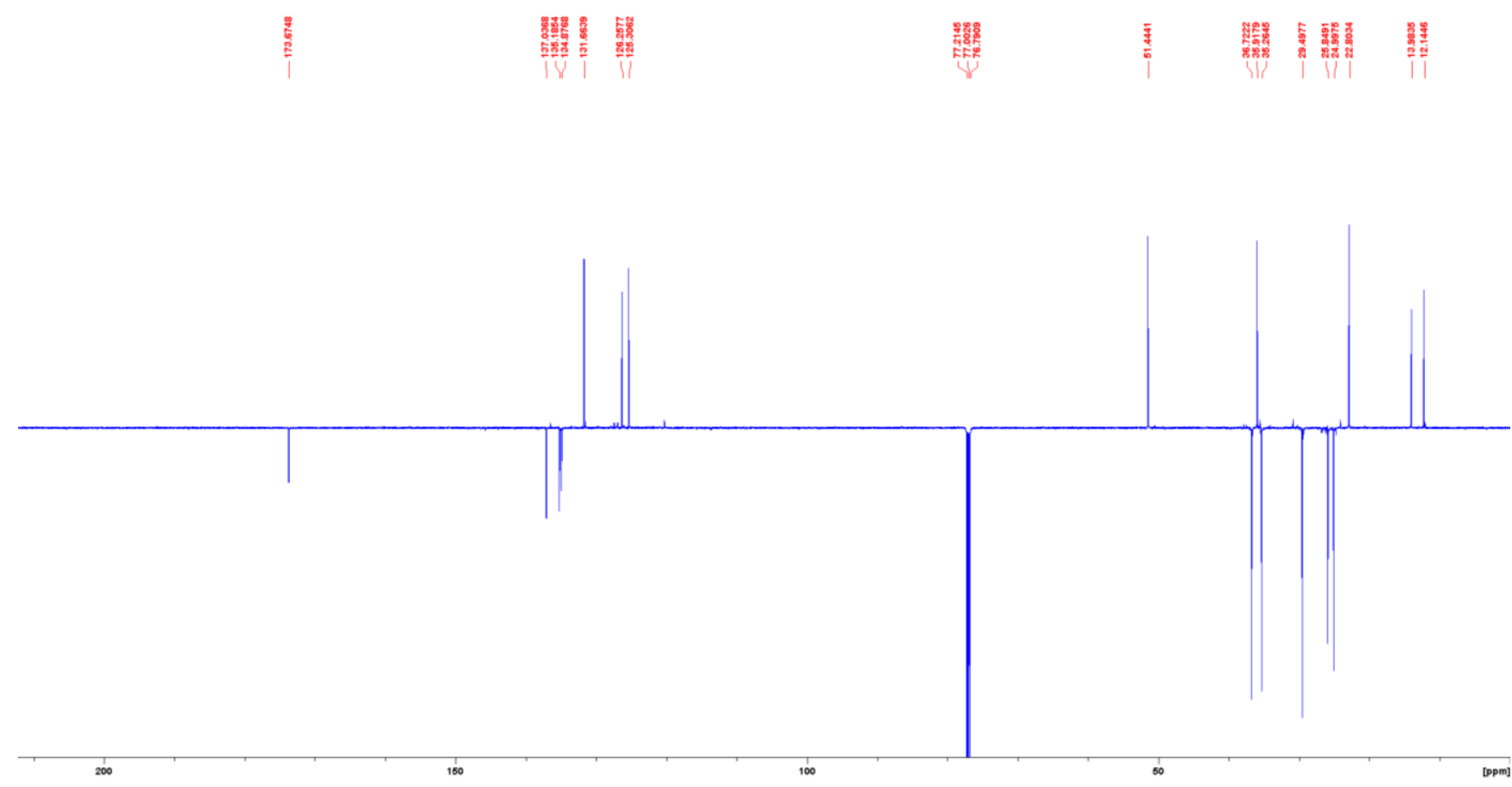


(rac)-2-[3-Methyl-2-((1E,3E)-3-methylpenta-1,3-dien-1-yl)cyclohept-2-en-1-yl]acetic acid (rac-9)

${ }^{1} \mathrm{H} \mathrm{NMR}\left(\mathrm{CDCl}_{3}, 400 \mathrm{MHz}\right)$

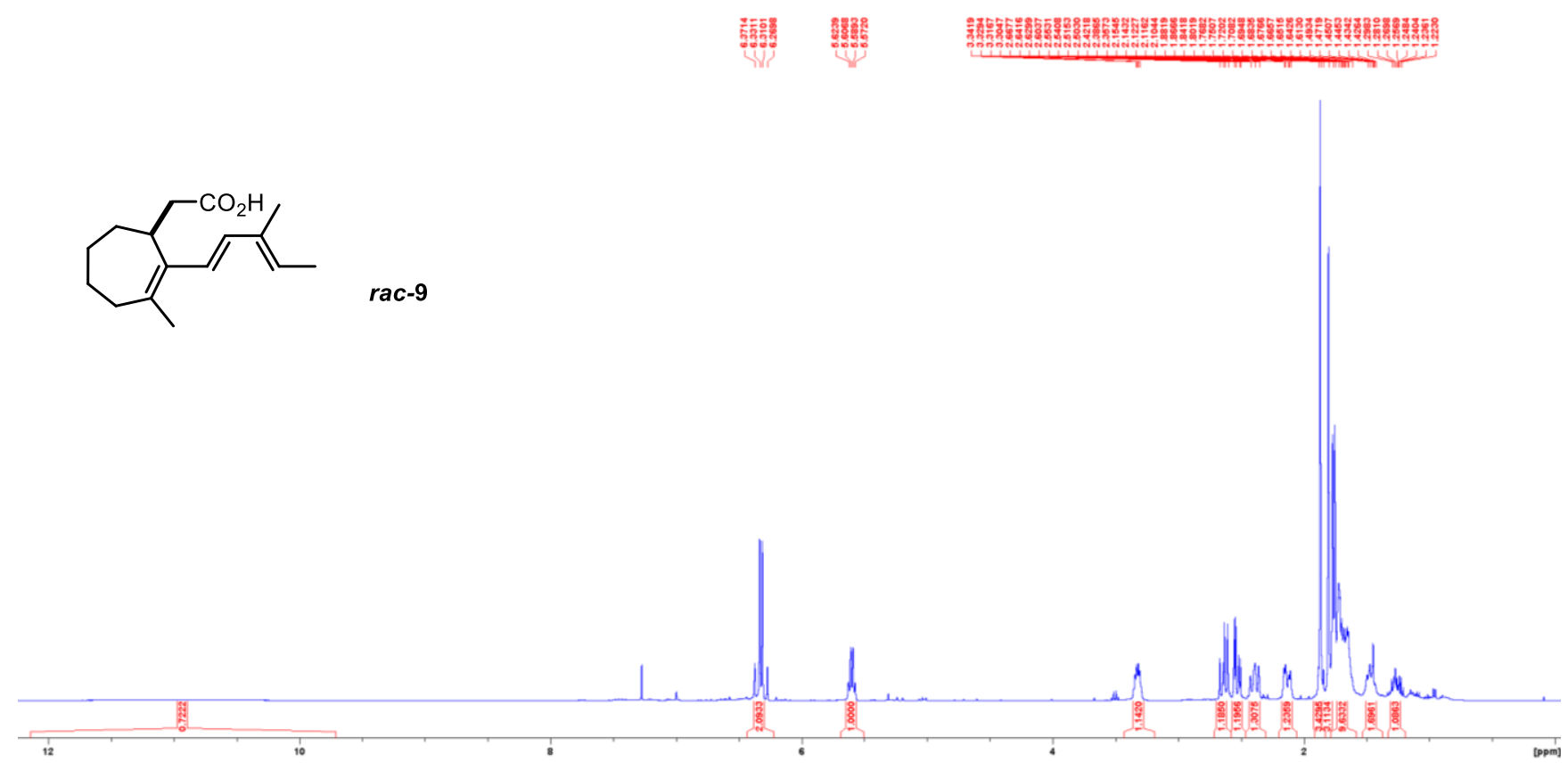

${ }^{13} \mathrm{C} \mathrm{NMR} \mathrm{(CDCl}, 100 \mathrm{MHz}$, DEPTQ experiment)

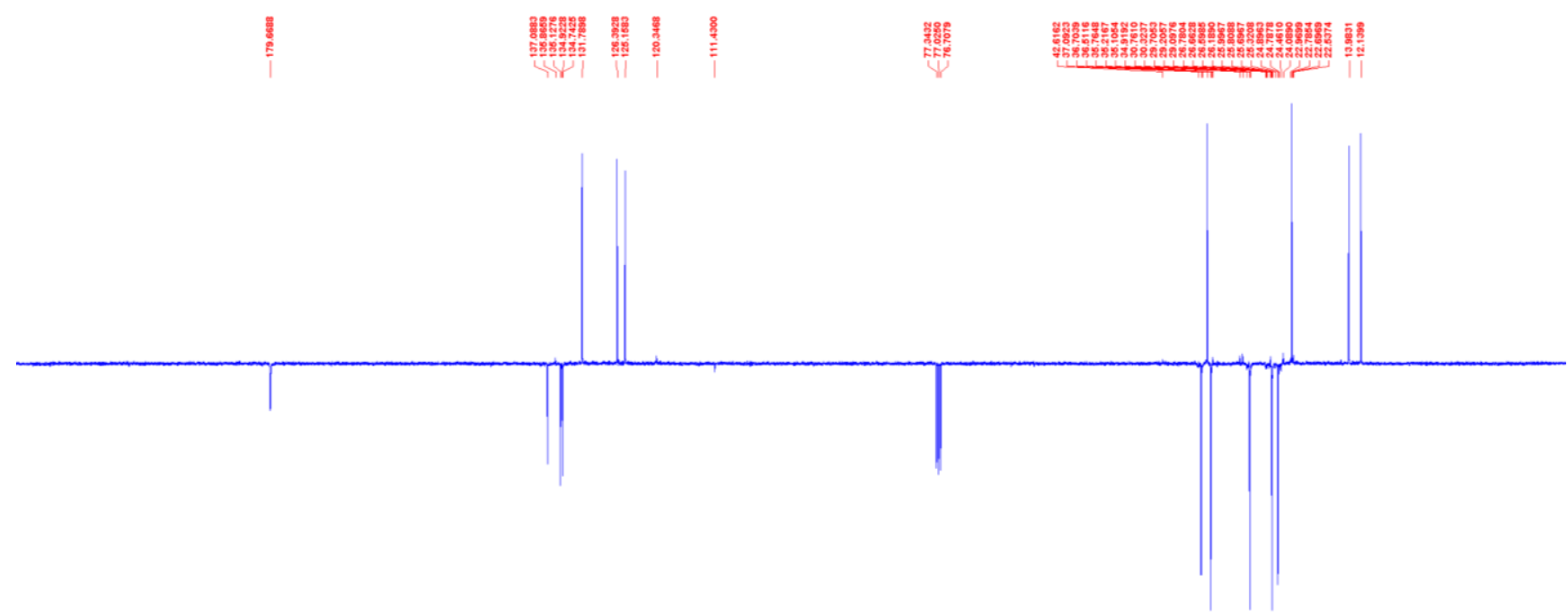


2-[(1S)-3-Methyl-2-((1E,3E)-3-methylpenta-1,3-dien-1-yl)cyclohept-2-en-1-yl]acetic acid, crude extract used in the next step (9)

${ }^{1} \mathrm{H}$ NMR $\left(\mathrm{CDCl}_{3}, 400 \mathrm{MHz}\right)$

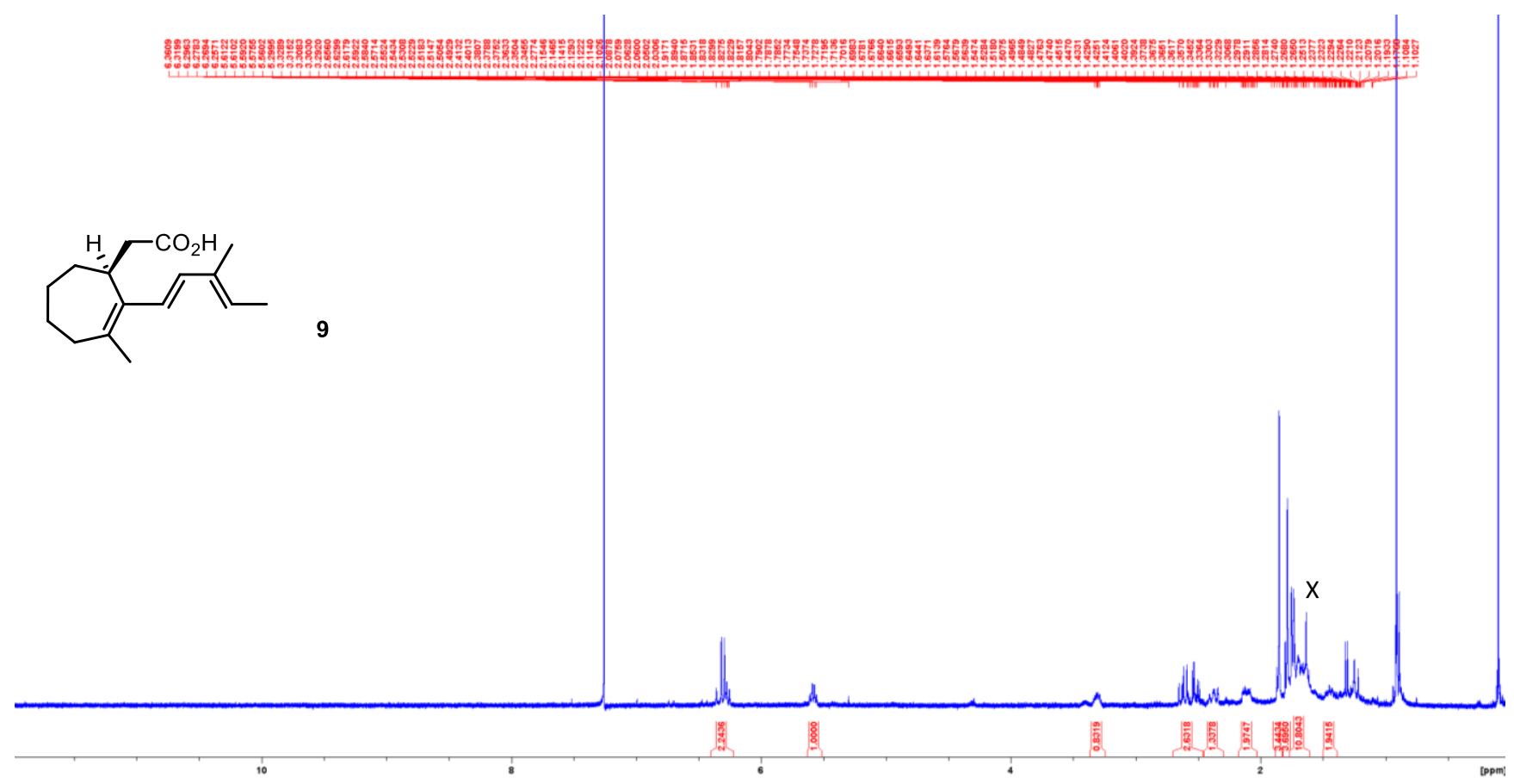

${ }^{13} \mathrm{C} \mathrm{NMR}\left(\mathrm{CDCl}_{3}, 100 \mathrm{MHz}\right)$

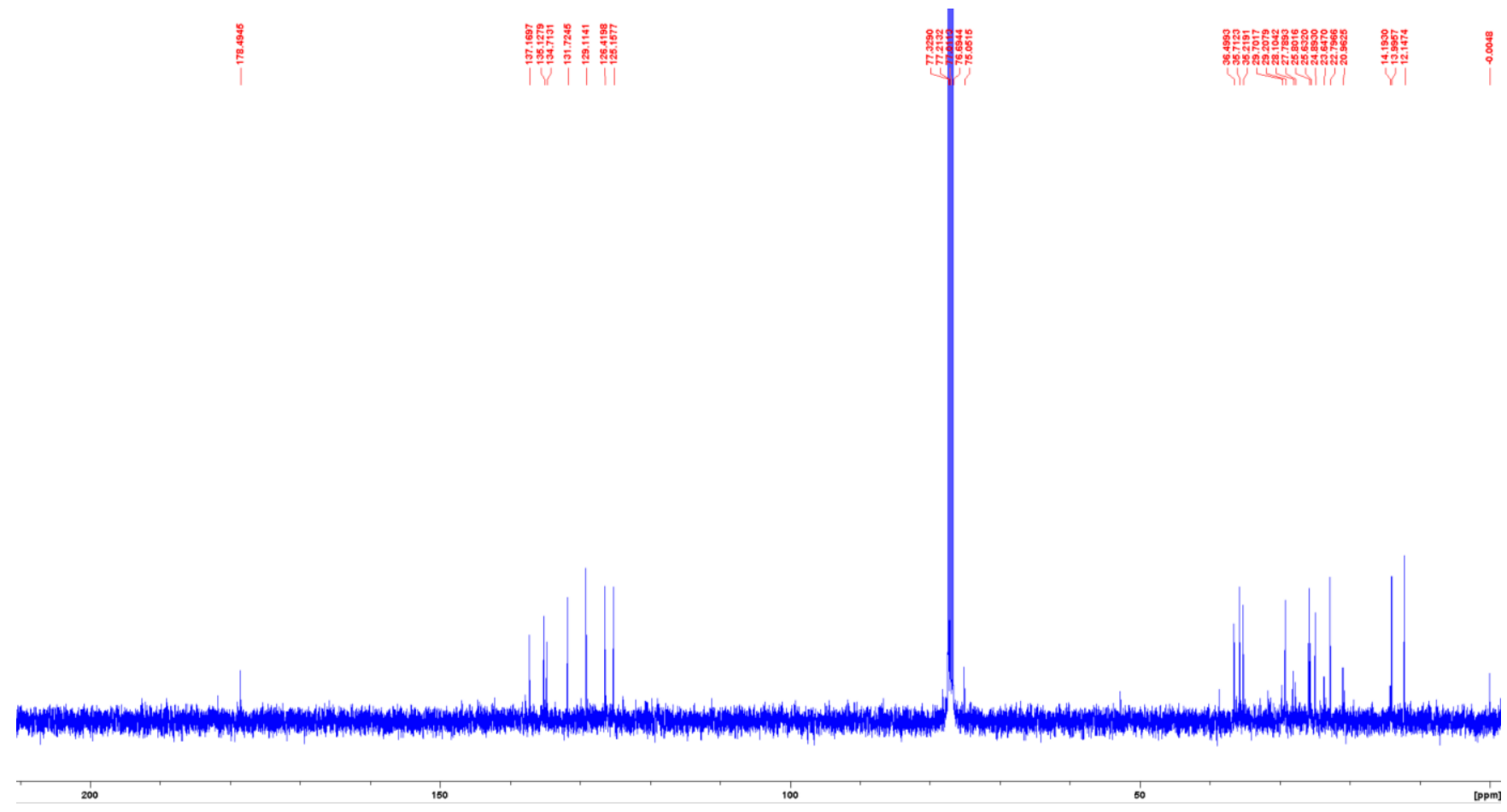


N-Benzoyl-5-isobutyl-3-\{2-[(1S)-3-methyl-2-((1E,3E)-3-methylpenta-1,3-dien-1-yl)cyclohept-2-en-1yl]acetyl\}-2-pyrrolidone, mixture of stereoisomers (22)

${ }^{1} \mathrm{H} \mathrm{NMR}\left(\mathrm{CDCl}_{3}, 400 \mathrm{MHz}\right)$

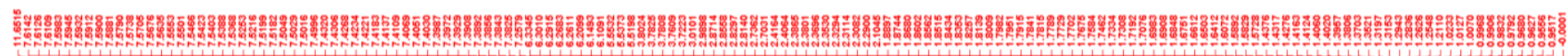<smiles>C/C=C(C)\C=C\C1=C(C)CCCCC1CC(=O)C1CC(CC(C)C)NC1=O</smiles>

22

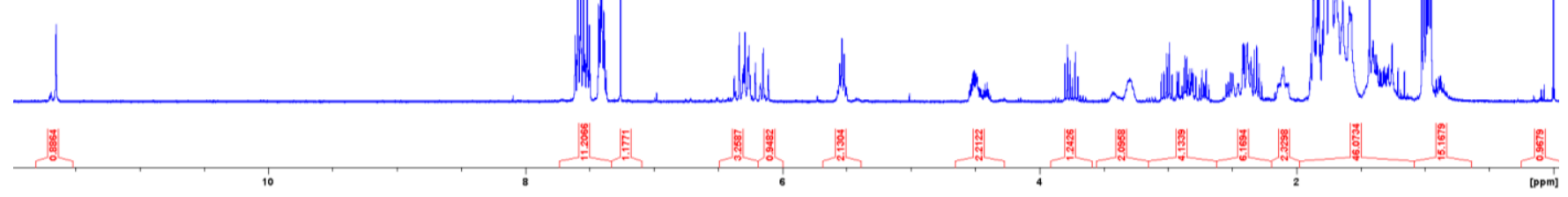

${ }^{13} \mathrm{C} \mathrm{NMR}\left(\mathrm{CDCl}_{3}, 100 \mathrm{MHz}\right), \mathrm{JMOD}$
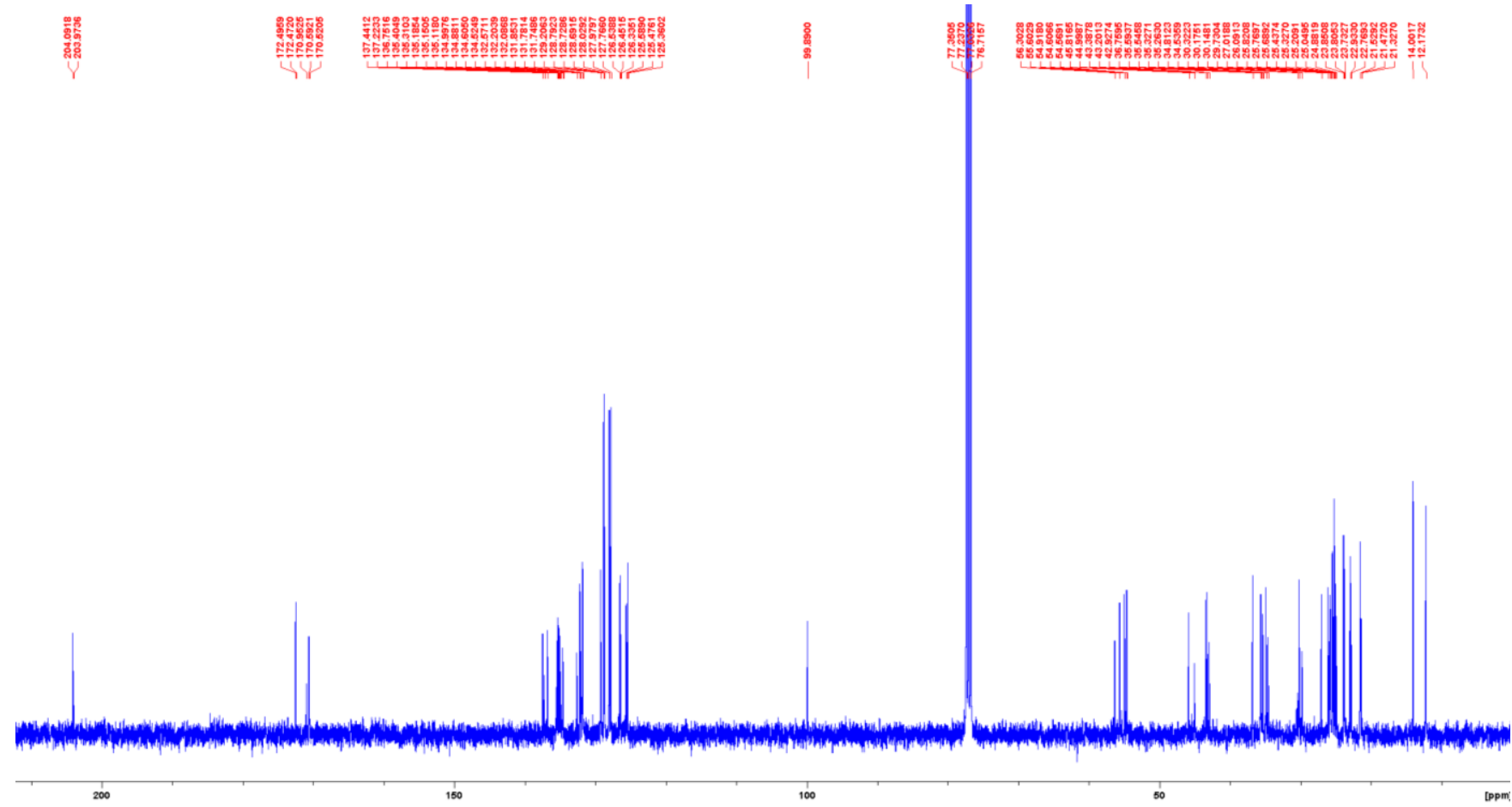
N-Benzoyl-5-isobutyl-3-\{2-[(1S)-3-methyl-2-((1E,3E)-3-methylpenta-1,3-dien-1-yl)cyclohept-2-en-1yl]acetyl\}-3-phenylselenyl-2-pyrrolidone, mixture of stereoisomers (23)

${ }^{1} \mathrm{H}$ NMR $\left(\mathrm{CDCl}_{3}, 400 \mathrm{MHz}\right)$

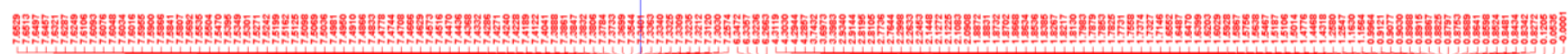

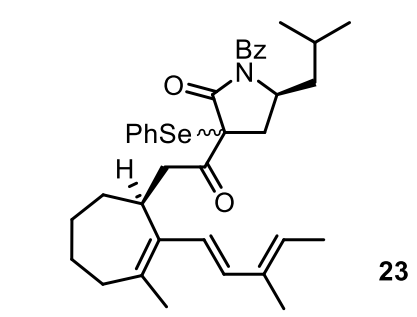

23

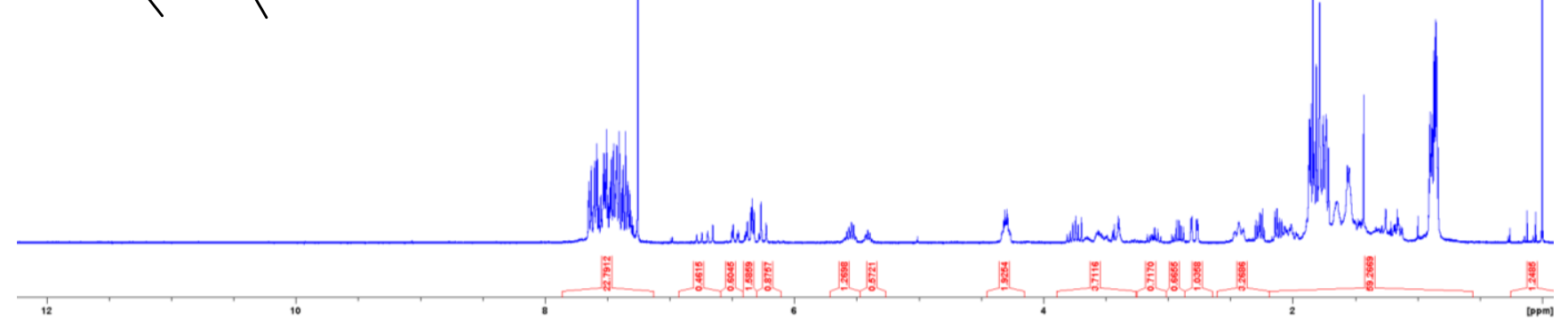

${ }^{13} \mathrm{C} \mathrm{NMR}\left(\mathrm{CDCl}_{3}, 100 \mathrm{MHz}\right)$

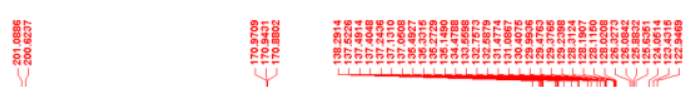
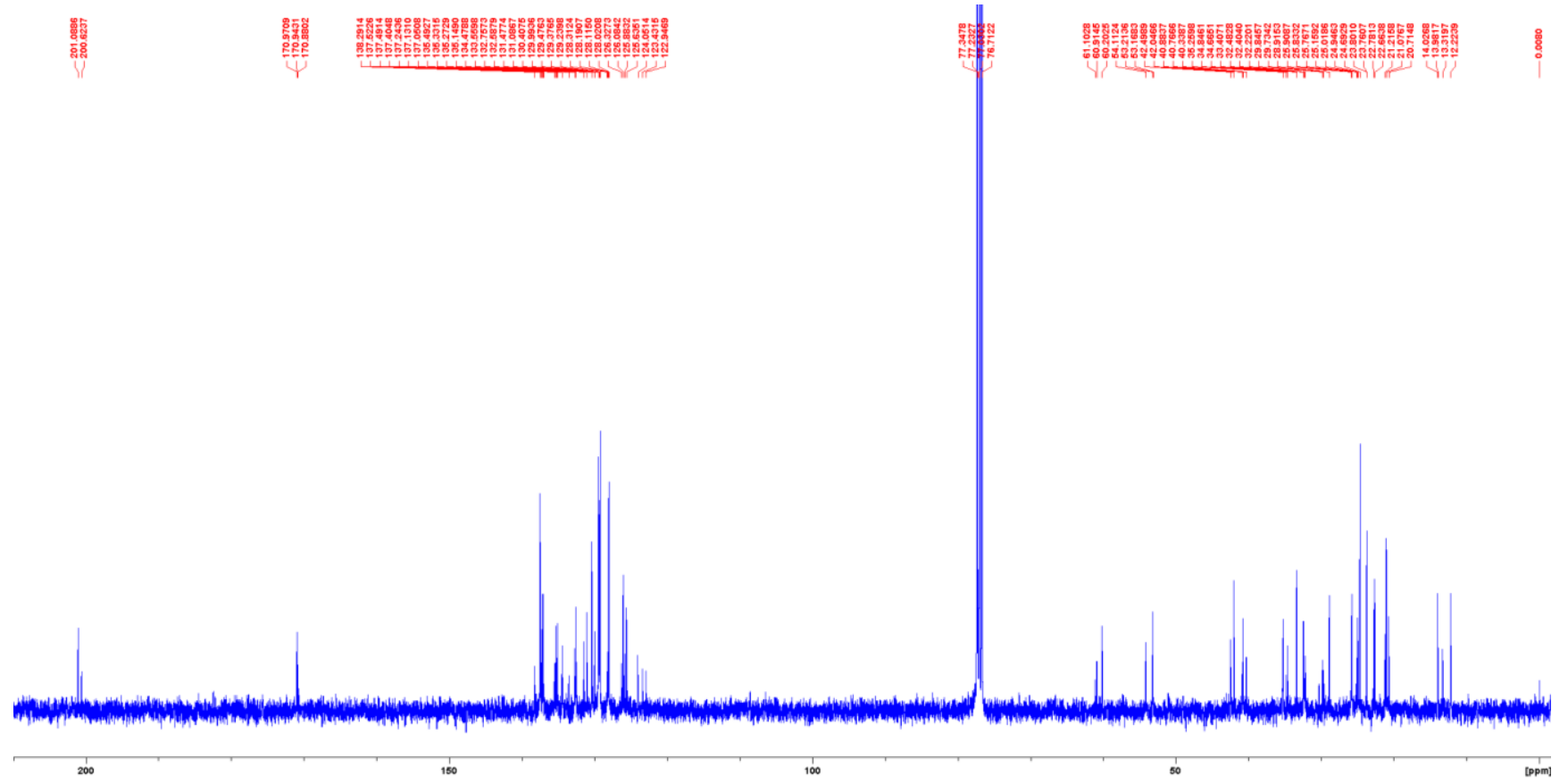
(3S,3aR,4S,6aS,8aS,13bR)-5-Benzoyl-4-isobutyl-2,3,13-trimethyl-4,5,8,8a,9,10,11,12-octahydro-3Hcyclohepta[3,4]benzo[1,2-d]isoindole-6,7(3aH,13bH)-dione (endo product 24)

${ }^{1} \mathrm{H} \mathrm{NMR}\left(\mathrm{CDCl}_{3}, 600 \mathrm{MHz}\right)$

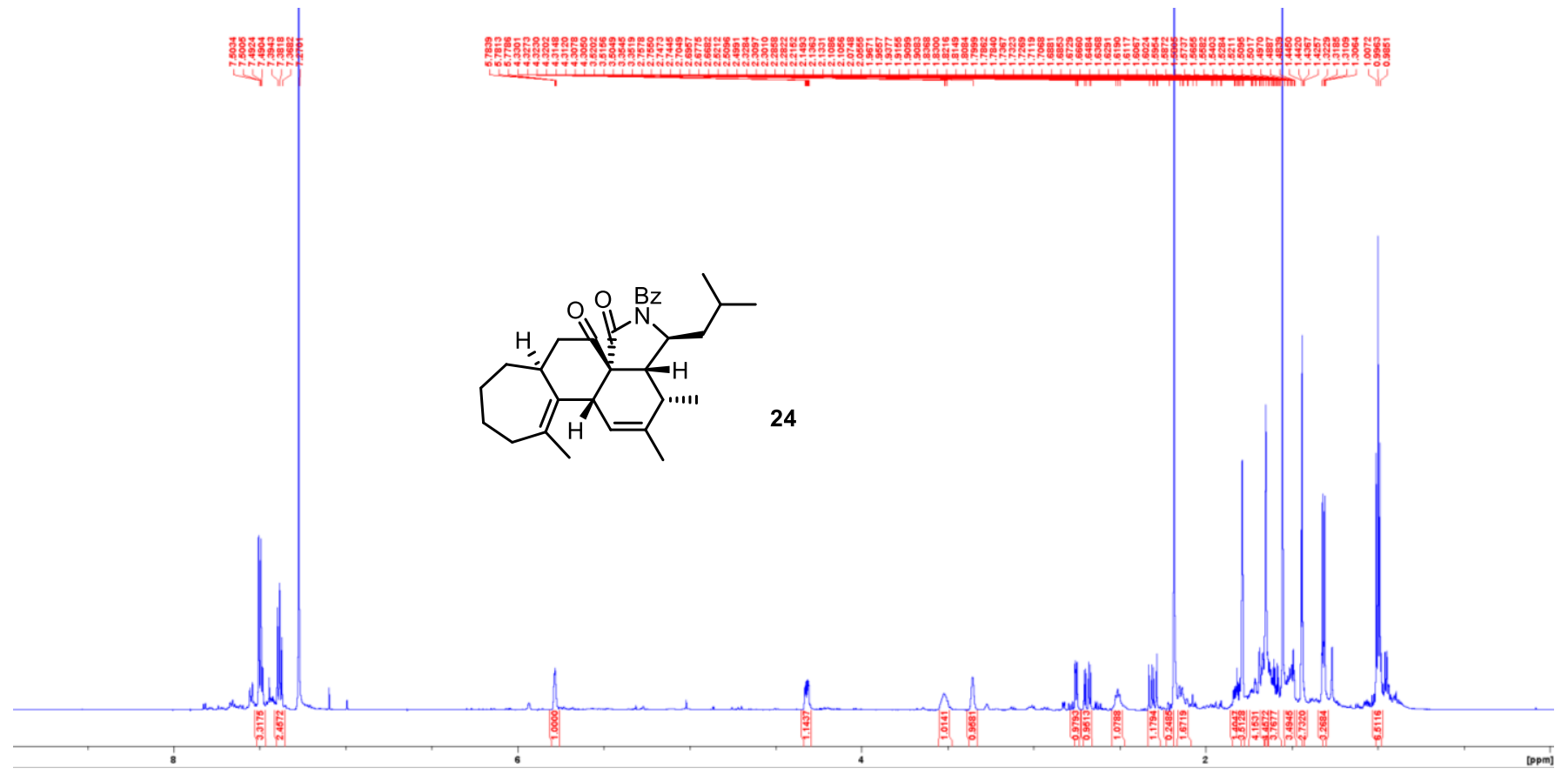

${ }^{13} \mathrm{C} \mathrm{NMR}\left(\mathrm{CDCl}_{3}, 150 \mathrm{MHz}\right)$, DEPTQ experiment
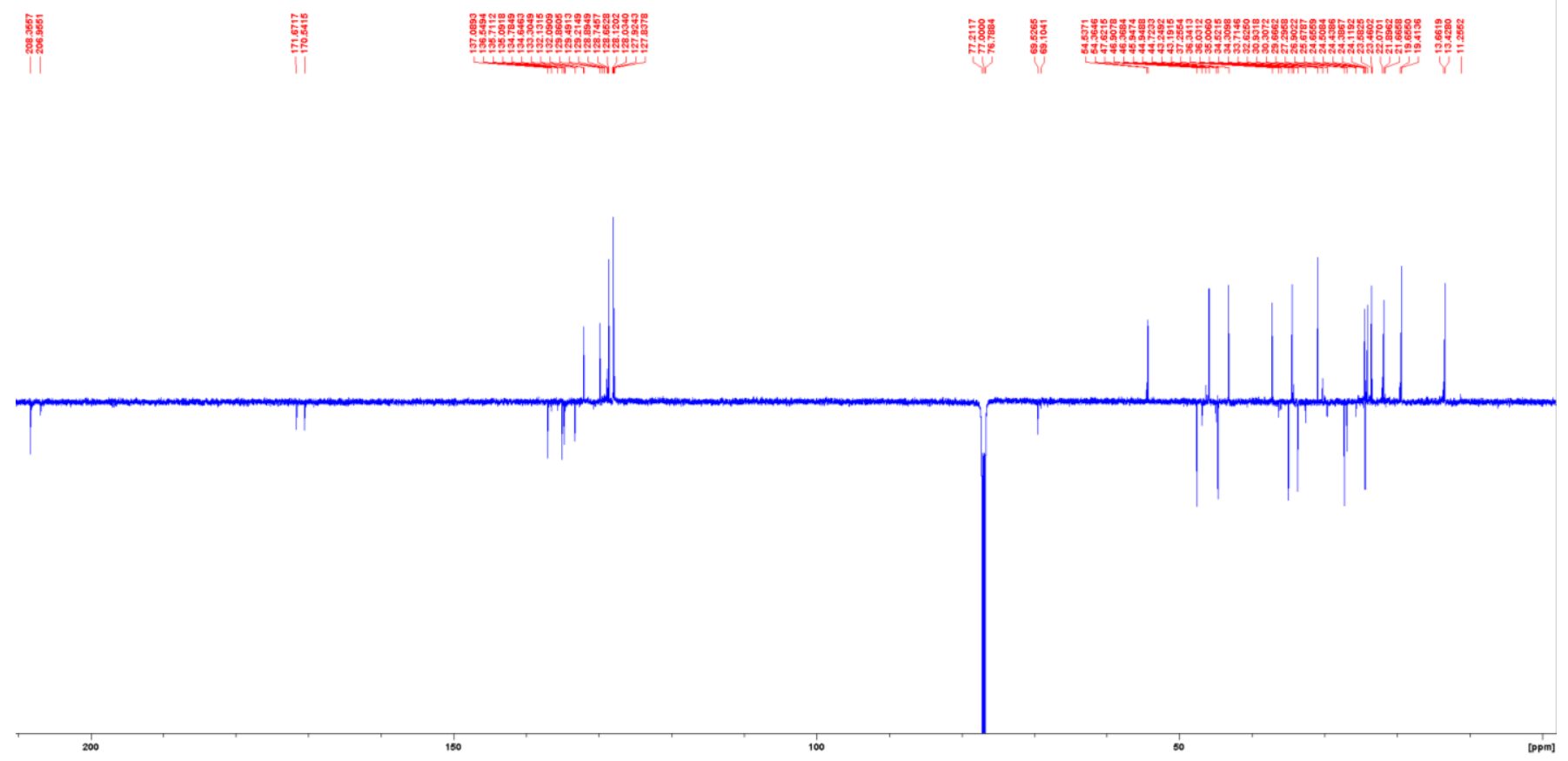
(3R,3aR,4S,6aS,8aS,13bS)-5-benzoyl-4-isobutyl-2,3,13-trimethyl-4,5,8,8a,9,10,11,12-octahydro-3Hcyclohepta[3,4]benzo[1,2-d]isoindole-6,7(3aH,13bH)-dione (exo product 25$)$

${ }^{1} \mathrm{H} \mathrm{NMR}\left(\mathrm{CDCl}_{3}, 600 \mathrm{MHz}\right)$

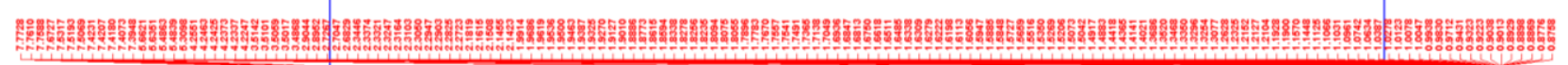

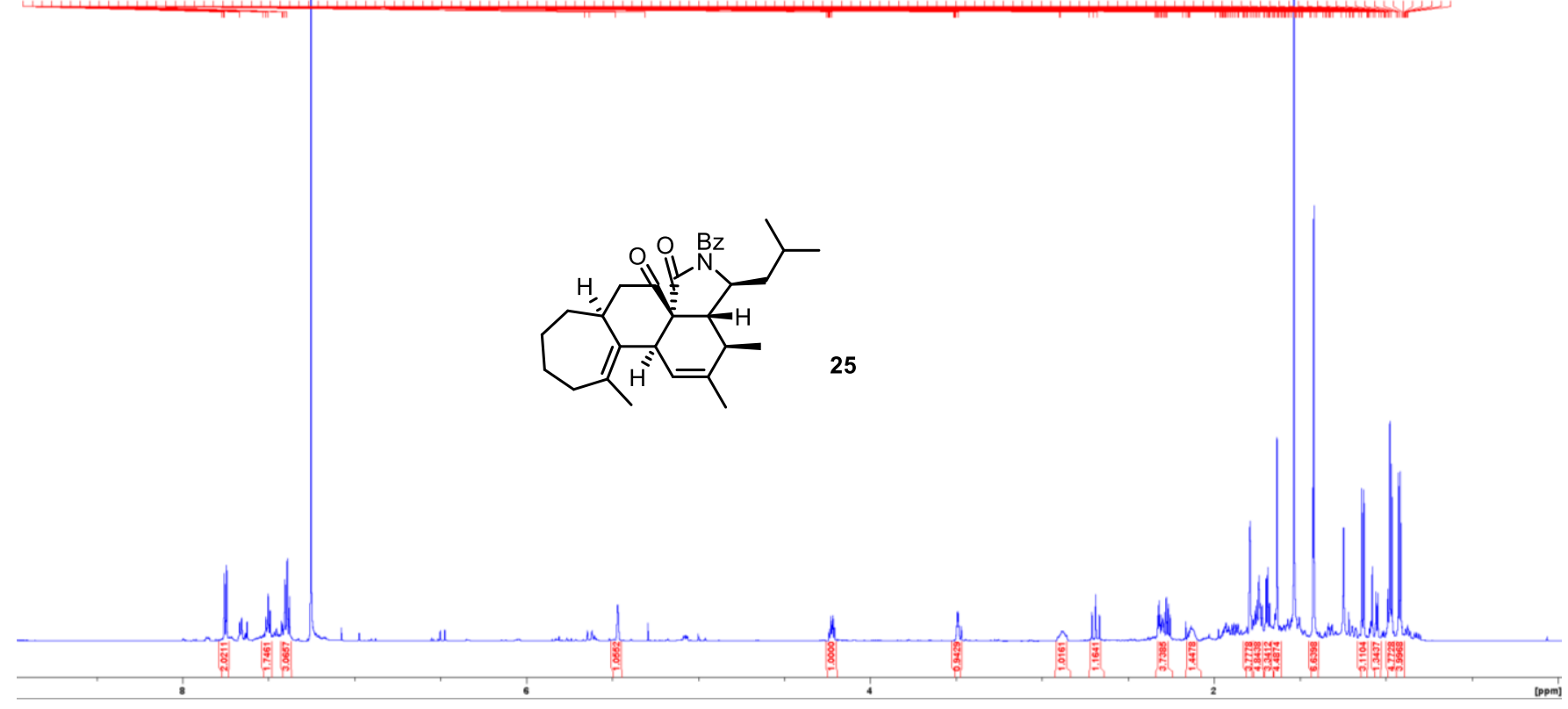

${ }^{13} \mathrm{C} \mathrm{NMR}\left(\mathrm{CDCl}_{3}, 150 \mathrm{MHz}\right)$, DEPTQ experiment

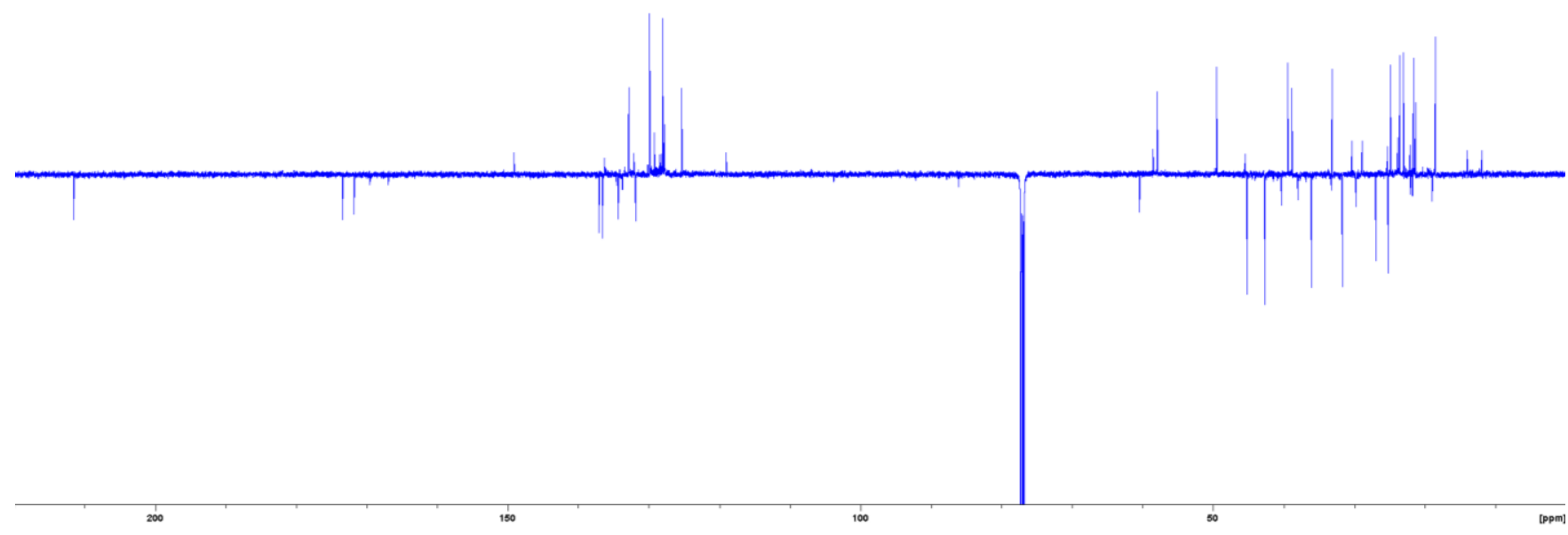


$(3 S, 3 a R, 4 S, 6 a S, 8 a S, 13 \mathrm{bR})-4-I s o b u t y l-2,3,13$-trimethyl-4,5,8,8a,9,10,11,12-octahydro-3Hcyclohepta[3,4]benzo[1,2-d]isoindole-6,7(3aH,13bH)-dione (biomimetic precursor 5)

${ }^{1} \mathrm{H} \mathrm{NMR}\left(\mathrm{CDCl}_{3}, 600 \mathrm{MHz}\right)$

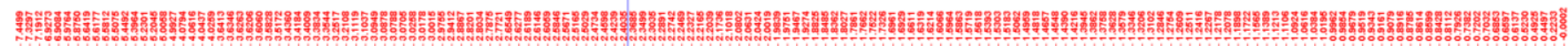

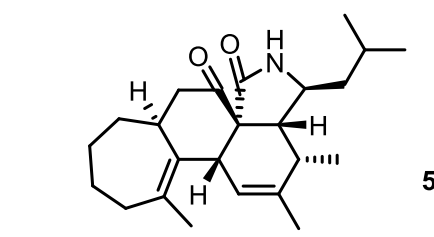

5

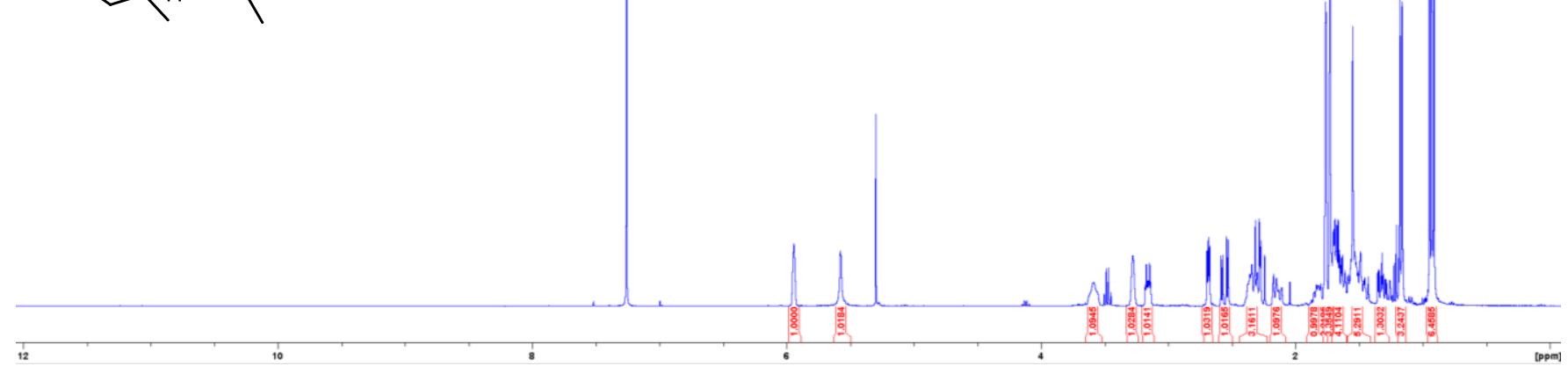

${ }^{13} \mathrm{C} \mathrm{NMR}\left(\mathrm{CDCl}_{3}, 150 \mathrm{MHz}\right)$, DEPTQ experiment
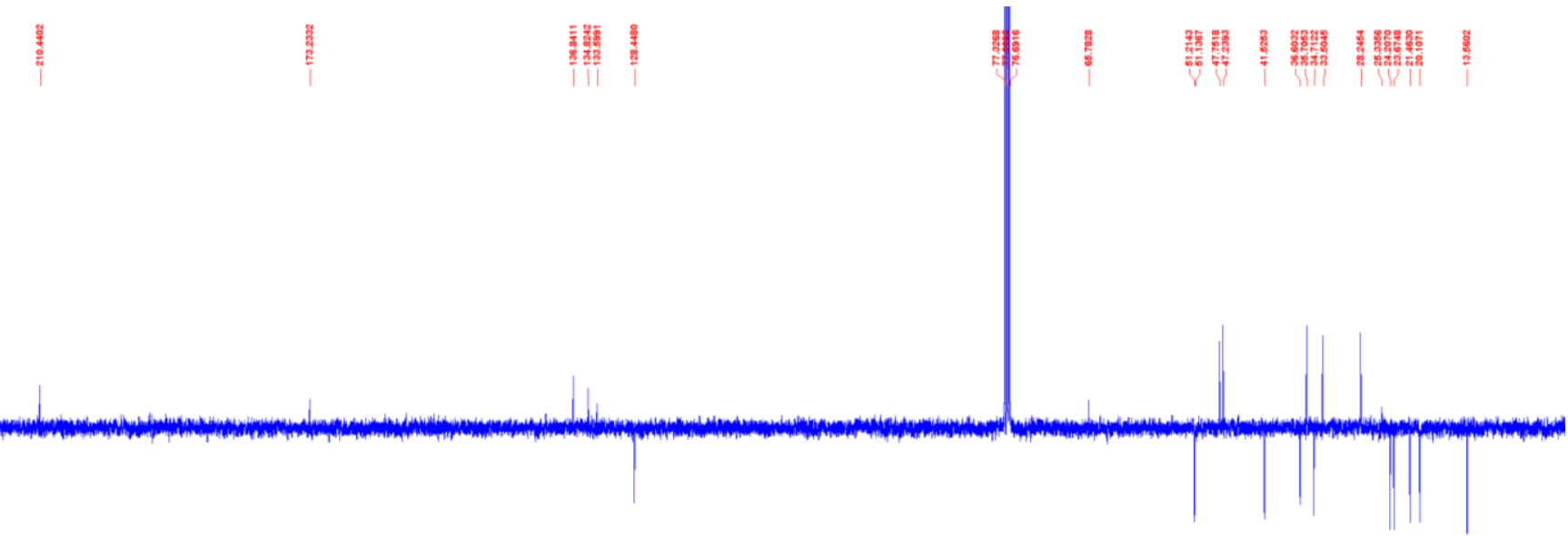
(3S,3aR,4S,6aS,8aS,13R,13aR,13bR)-5-Benzoyl-13,13a-epoxy-4-isobutyl-2,3,13-trimethyl4,5,8,8a,9,10,11,12-octahydro-3H-cyclohepta[3,4]benzo[1,2-d]isoindole-6,7(3aH,13bH)-dione (26)

${ }^{1} \mathrm{H}$ NMR $\left(\mathrm{CDCl}_{3}, 400 \mathrm{MHz}\right)$

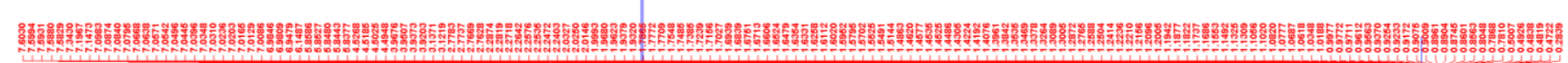

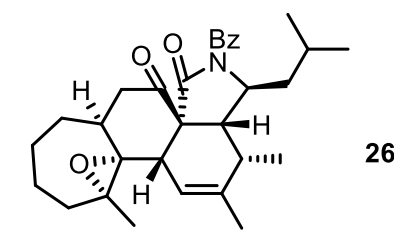

26

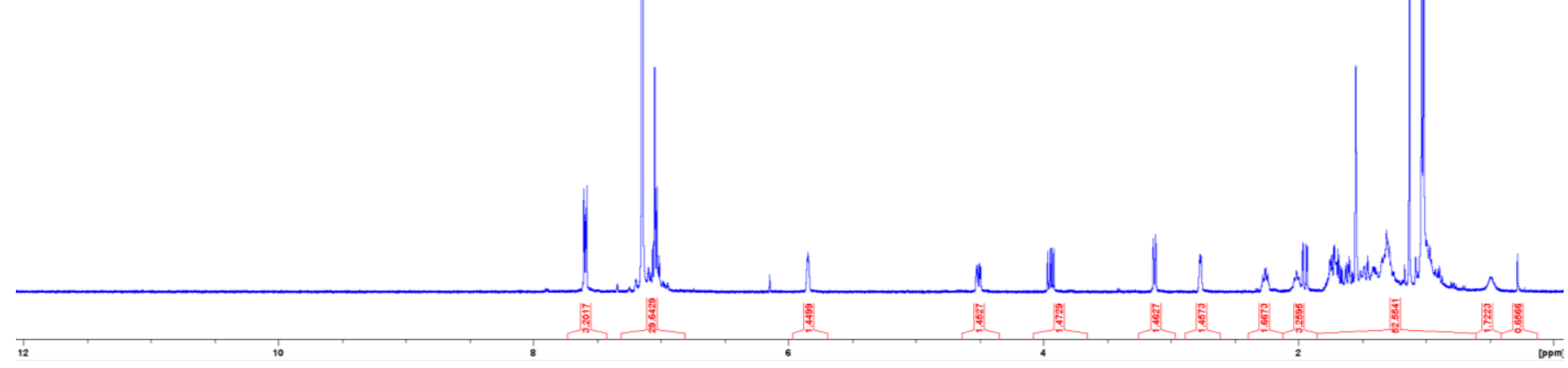

${ }^{13} \mathrm{C} \mathrm{NMR}\left(\mathrm{CDCl}_{3}, 100 \mathrm{MHz}\right)$, DEPTQ experiment

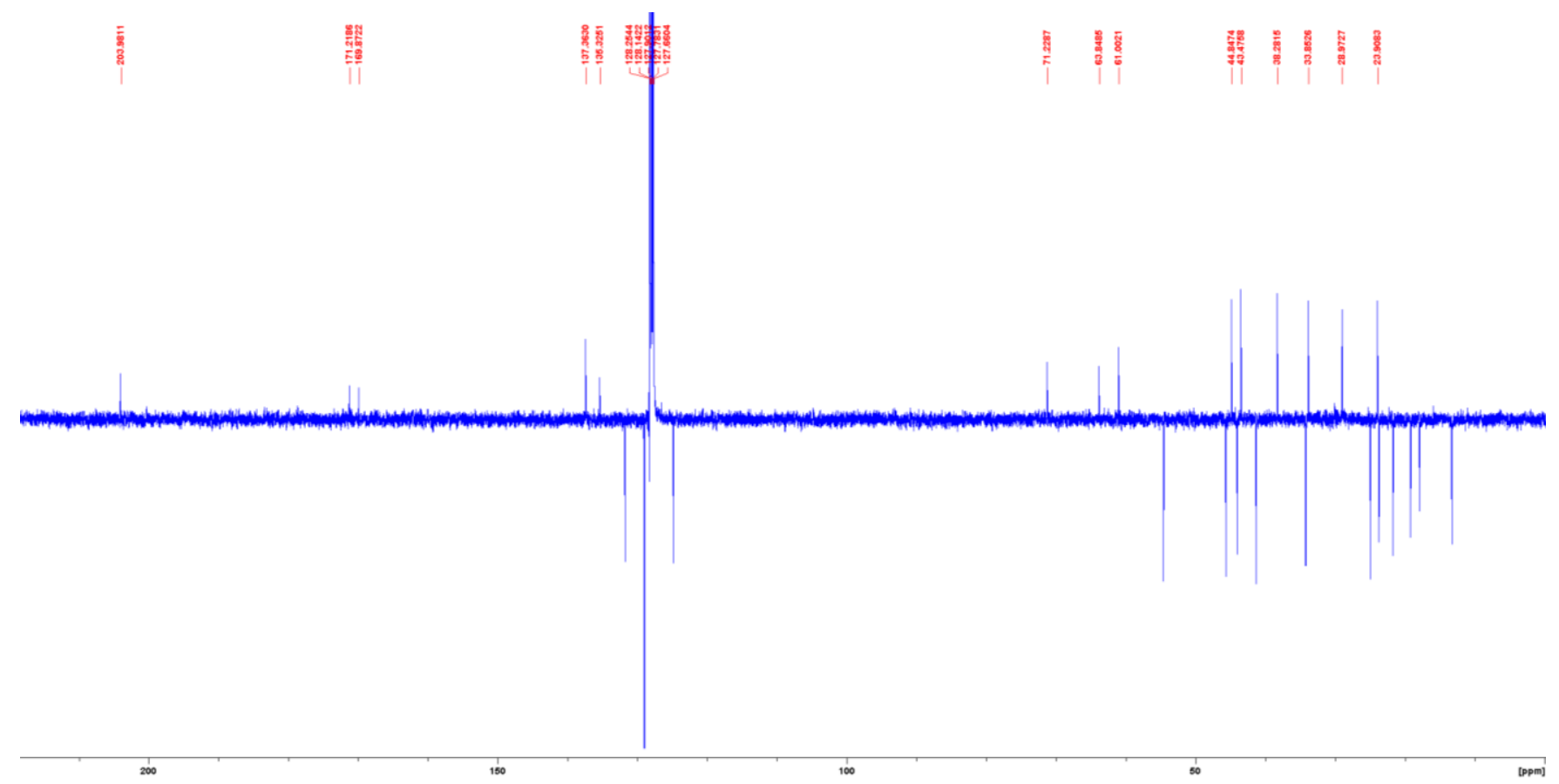

